\title{
Instance Segmentation of Point Clouds via Similarity Learning
}

\author{
by \\ Mehak Mustafa, B.Sc.

\begin{abstract}
A thesis submitted to the
Faculty of Graduate and Postdoctoral Affairs

in partial fulfillment of the requirements for the degree of
\end{abstract}

Master of Computer Science

Ottawa-Carleton Institute for Computer Science

The School of Computer Science

Carleton University

Ottawa, Ontario

July, 2020

(C)Copyright

Mehak Mustafa, 2020 
The undersigned hereby recommends to the

Faculty of Graduate and Postdoctoral Affairs acceptance of the thesis

\title{
Instance Segmentation of Point Clouds via Similarity Learning
}

\author{
submitted by Mehak Mustafa, B.Sc. \\ in partial fulfillment of the requirements for the degree of \\ Master of Computer Science
}

Professor Oliver van Kaick, Thesis Supervisor

Professor David Mould, School of Computer Science

Professor Jochen Lang,

School of Electrical Engineering and Computer Science

Professor Majid Komeili, Chair, School of Computer Science

Ottawa-Carleton Institute for Computer Science

The School of Computer Science

Carleton University

July, 2020 


\section{Abstract}

We introduce a novel approach for instance segmentation of point clouds without using instance label information. Our method makes use of a form of similarity learning with semantic information. Specifically, we modify the loss function of the PointNet [38] neural network so that the network can learn to predict point features, where the point features are close to each other in feature space if the points belong to the same semantic category. We call this network trained with a modified loss function PointSimNet, for brevity. PointSimNet is trained on a set of point clouds of rooms where objects are grouped into different categories.

After predicting features and semantic labels with PointSimNet, we perform spectral clustering to obtain instances. The spectral clustering method can be performed on features or coordinates. A similarity matrix, given by applying a Gaussian kernel to a matrix of feature distances or point coordinate distances, is used to find the optimal number of clusters using the eigengap heuristic. Spectral clustering is then performed on the similarity matrix to obtain the final set of instances.

We also provide an optional step of constrained clustering where the user can guide the clustering to refine the segmentation results. In this step, the user provides the desired number of clusters and one or more constraints where the user identifies points that cannot be linked together.

We show that our instance segmentation method provides 16 percentage points lower than a state-of-the-art method in terms of average precision, but outperforms the method by 20 percentage points according to an alternative definition of the average precision. We are able to achieve these results without requiring any instance information in the training set. We also demonstrate that the user can improve the accuracy of results by adding a small number of constraints. 


\section{Acknowledgments}

First and foremost, I would like to acknowledge my supervisor, Oliver Van Kaick, for his guidance and support during this journey. He gave me the opportunity to not only expand my knowledge in the field of machine learning but also create an innovative algorithm to make an impact in the research community. His constant encouragement and teaching has resulted in the completion of this part of the research.

I would also like to thank Professor David Mould, Professor Jochen Lang and Professor Michel Barbeau for providing valuable feedback and improvements for my thesis. I would like to thank my lab members Yanran Guan and Tansin Jahan for their assistance in experiments and visualization.

Next, I would like to thank Professor Stephen Fai, the director of Carleton Immersive Media Studio (CIMS), for introducing this problem to Oliver and me. Because of this, we have been able to make a joint effort from the field of Architecture and Computer Science. Everyone at CIMS has been a huge help during this time and this project would not be what it is today without their support.

I am immensely grateful to my greatest support during my entire life, my parents. They have provided me with financial, emotional and physical support with all endeavors and I hope to make them proud with my achievements.

Lastly, I would like to thank School of Computer Science for providing the financial support i needed to complete my masters. I appreciate the computer Science faculty and staff for making this a pleasant ride for me. 


\section{Table of Contents}

Abstract

Acknowledgments $\quad$ iv

Table of Contents $\quad$ v

List of Tables $\quad$ vii

List of Figures $\quad$ ix

1 Introduction $\quad 1$

1.1 Motivation of the thesis . . . . . . . . . . . . . 1

1.2 Objective of the thesis . . . . . . . . . . . . . . . . 3

1.3 Overview of the method . . . . . . . . . . . . 4

1.4 Summary of contributions . . . . . . . . . . . . . 6

1.5 Organization of the thesis . . . . . . . . . . . 6

2 Background and literature review $\quad 8$

2.1 Machine Learning . . . . . . . . . . . . . . . . . 8

2.1.1 Supervised Machine Learning . . . . . . . . . . . . . . 9

2.1.2 Unsupervised Machine Learning . . . . . . . . . . . . . . . . . 12

2.1.3 Semi-Supervised Machine Learning . . . . . . . . . . . . . 13

2.2 Point Clouds . . . . . . . . . . . . . . . . . . 15

2.2.1 Point Cloud Problems and Solution methods . . . . . . . . . . 16

2.3 Point Cloud Processing with Machine Learning . . . . . . . . . . . . 18

3 Instance Segmentation method 24

3.1 Data assumptions . . . . . . . . . . . . . . . . 25 
3.2 Learning of Similarity measure . . . . . . . . . . . . . . . . . . . 25

3.3 Learning of Semantic labels . . . . . . . . . . . . . . . . . . . 28

3.4 Composing a Similarity matrix . . . . . . . . . . . . . . 30

3.5 Clustering the Similarity matrix . . . . . . . . . . . . . . . 31

3.5.1 Finding the number of Clusters . . . . . . . . . . . . . 33

3.6 Constrained Clustering . . . . . . . . . . . . . . . 34

3.7 Summary . . . . . . . . . . . . . . . . . 37

4 Results and Evaluation 38

4.1 Datasets . . . . . . . . . . . . . . . . . . . 38

4.1 .1 Stanford Dataset (S3DIS) . . . . . . . . . . . 38

4.1 .2 CIMS Dataset . . . . . . . . . . . . . . . . . . 39

4.2 Training and Parameters . . . . . . . . . . . . . . . . . . 39

4.3 Evaluation measures . . . . . . . . . . . . . . . . . . . 40

4.4 Quantitative results . . . . . . . . . . . . . . . 45

4.5 Qualitative results ..................... 51

4.5.1 Semantic Segmentation results . . . . . . . . . . . . . 51

4.5.2 Instance Segmentation results . . . . . . . . . . . . . . . . . 54

4.6 Constrained Clustering Evaluation

(Quantitative and Qualitative) . . . . . . . . . . 59

4.7 Execution time . . . . . . . . . . . . . . . 69

$\begin{array}{lll}5 & \text { Conclusion } & 70\end{array}$

5.1 Discussion . . . . . . . . . . . . . . . . . . 70

5.2 Limitations . . . . . . . . . . . . . . . . . . . . . . 71

5.3 Future work . . . . . . . . . . . . . . . . . . . . 72

$\begin{array}{ll}\text { List of References } & 74\end{array}$ 


\section{List of Tables}

4.1 Statistics for 10 different S3DIS rooms showing the number of bounding boxes computed with the minimum and maximum versus the mean and standard deviation. "Percentage" denotes the percentage of boxes out of the total computed with the minimum and maximum. . . . . . . . 42

4.2 Semantic segmentation results on S3DIS and CIMS datasets. These values represent the labeling accuracy, which is the number of correctly predicted points divided by the total number of points. . . . . . . . .

4.3 Semantic segmentation results on S3DIS. The measure is the average IoU over 13 classes. . . . . . . . . . . . . . . . . . . . . . . .

4.4 Instance segmentation results on the S3DIS dataset. The metric is $\mathrm{AP} \%$ with IoU of 0.5 , and is calculated on sets of points as introduced by SGPN. . . . . . . . . . . . . . . . . . .

4.5 Instance segmentation results on the S3DIS dataset. The metric is $\mathrm{AP} \%$ with IoU of 0.5 , and is calculated using the bounding box method. 46

4.6 Instance segmentation results on the S3DIS dataset. The metric is $\mathrm{AP} \%$ with different IoU thresholds, and is calculated on sets of points over 12 categories.

4.7 Instance segmentation results on the S3DIS dataset. The metric is AP\% with different IoU thresholds, and is calculated using the bounding box method over 12 categories. . . . . . . . . . . . .

4.8 Instance segmentation results on the S3DIS dataset for different rooms. The metric is $\mathrm{AP} \%$ with IoU threshold of 0.5, and is calculated using sets of points. . . . . . . . . . . . . . . . .

4.9 Instance segmentation results on the S3DIS dataset for different rooms. The metric is $\mathrm{AP} \%$ with IoU threshold of 0.5 , and is calculated using the bounding box method. . . . . . . . . . . . . . . . . 
4.10 Instance segmentation results on the S3DIS dataset on Area 5. The method marked with $*$ is evaluated with 6 -fold cross validation. The metrics are mPrec and mRecall with IoU threshold of 0.5 , calculated using the point set method. . . . . . . . . . . . . . .

4.11 Instance segmentation results on the S3DIS dataset showing experiments with different $\delta$ values in gaussian kernel. The metric is AP\% with IoU of 0.5 , and is calculated using the bounding box method. . .

4.12 Instance segmentation results on Office 2 from the S3DIS dataset. The metric is AP\% with IOU of 0.5 and is calculated using the point method. 60

4.13 Instance segmentation results on Office 8 from the S3DIS dataset. The metric is $\mathrm{AP} \%$ with IOU of 0.5 and is calculated using the point method. 61

4.14 Instance segmentation results on Office 2 from the S3DIS Dataset. The metric is $\mathrm{AP} \%$ with IOU of 0.5 and is calculated using the bounding box method. . . . . . . . . . . . . . . . . . . .

4.15 Instance segmentation results on Office 8 from the S3DIS dataset. The metric is $\mathrm{AP} \%$ with $\mathrm{IOU}$ of 0.5 and is calculated using the bounding box method. . . . . . . . . . . . . . . . . . . . . . . 61

4.16 Instance segmentation results on the S3DIS dataset for Office 8. The metrics are mean precision and mean recall with IoU threshold of 0.5 , which are calculated using the point set method. . . . . . . . . . .

4.17 Instance segmentation results on the S3DIS dataset for Office 2. The metrics are mean precision and mean recall with IoU threshold of 0.5 , which are calculated using the point set method. . . . . . . . . . 62

4.18 Execution time analysis for our method. . . . . . . . . . . . . . . . 69 


\section{List of Figures}

1.1 Overview of our method. . . . . . . . . . . . . . . . . 4

2.1 Difference between semantic segmentation and instance segmentation. 17

2.2 PointNet architecture, where MLP denotes "multi-layer perceptron network". Image source: Qi et al. [38]. . . . . . . . . . . . . . . 18

2.3 SGPN architecture. Image source: Wang et al. [50]. . . . . . . . . . . 21

3.1 Overview of our method for instance segmentation. . . . . . . . . 24

3.2 Comparison of the similarity of point features of a chair predicted by PointSimNet (center) and PointNet (right). The input point cloud is shown on the left. . . . . . . . . . . . . . . . 27

3.3 Comparison of the similarity of point features of a door predicted by PointSimNet (center) and PointNet (right). The input point cloud is shown on the left. . . . . . . . . . . . . . . . 27

3.4 Overview of the T-Net Process. Image source of Luis Gonzales [18]. . 29

3.5 PointNet architecture. Image source: Qi et al. [38]. . . . . . . . . . 30

3.6 Eigengap heuristic illustration. Image source: Von Luxburg [47]. . . . 34

3.7 Use of the Lasso tool to circle points for cannot-link constraints. . . 35

3.8 Final output of constrained clustering for chairs. . . . . . . . 36

3.9 Benefit of constrained clustering. . . . . . . . . . . . 36

4.1 Semantic segmentation results on the S3DIS dataset. . . . . . . . 52

4.2 Semantic segmentation results on the CIMS dataset. . . . . . . . 53

4.3 Semantic segmentation results on the CIMS dataset with the model trained on the S3DIS dataset. . . . . . . . . . . . . . 54

4.4 Instance segmentation results for Office $6 \ldots \ldots \ldots \ldots$

4.5 Instance segmentation results for Office $32 \ldots \ldots \ldots \ldots$

4.6 Instance segmentation results for Office $22 \ldots \ldots \ldots \ldots$

4.7 Instance segmentation results for Office $31 \ldots \ldots \ldots \ldots \ldots$

4.8 Instance segmentation results for Office $29 \ldots \ldots \ldots \ldots$ 
4.9 Instance segmentation results on CIMS dataset. . . . . . . . . . . . 59

4.10 Constrained clustering results for walls of Office 2 . . . . . . . . . 63

4.11 Office 2 results for instance segmentation. The second columns on the first and second rows show the result on the left improved by manually providing the number of clusters. . . . . . . . . . . . . .

4.12 Office 8 results for instance segmentation. The second columns on the first and second rows show the result on the left improved by manually providing the number of clusters. . . . . . . . . . . . . .

$4.13601 \mathrm{~S}$ results for instance segmentation. The second columns on the first and second rows show the result on the left improved by manually providing the number of clusters. . . . . . . . . . . . . 66

4.14 Room 484S results for instance segmentation. The second columns on the first and second rows show the result on the left improved by manually providing the number of clusters. . . . . . . . . . . . 


\section{Chapter 1}

\section{Introduction}

\subsection{Motivation of the thesis}

Before the construction of any architectural project, a planning phase consists of assessing different variables attached to the project. This can include the type of tools and materials, the number of people, and the time required to carry out the construction. Creating a digital model of a building prior to the construction or renovation of the building can help in planning and possibly simulating the work to be carried out. The different physical infrastructure modules within a building require an accurate model representation to make optimal decisions.

In this context, building information modeling is the process of creating digital models for new buildings and construction projects, which are then called Building Information Models (BIMs) [6]. BIMs enable the planning and management of the project. Before the existence of BIMs, architects used to create 2D drawings on paper to present their ideas about a building and their plans related to it. This $2 \mathrm{D}$ representation gave way to BIMs, which are models that can represent not only the 3D geometry of a building, but also incorporate time/use as a fourth dimension. BIMs can be used in the planning phase of the construction of a building and during the life-cycle of a building, such as for management and operation of the building, which can include operational cost management, maintenance and renovation.

Currently, at the start of any major construction project, a BIM of the building is created. Any buildings that have been built before the existence of BIMs and are considered heritage sites are documented using Heritage Building Information Models (HBIMs). HBIMs are dedicated to the modeling of cultural heritage sites, and aid in sustenance and preservation of these sites. HBIMs record the different assets within a 
heritage building and can thus help in tracking these assets, e.g., furniture, art works, architectural designs such as windows and doors. This is useful as these valuable assets may be moved and/or renovated during a rehabilitation project. The use of HBIMs can help in reducing the human effort needed as well as the cost and risk associated with rehabilitation projects. HBIMs for heritage buildings are only constructed when a rehabilitation project starts due to the site predating existing technology. As most of the heritage assets contain complex structures that are different from current designs, human input and a lot of time is dedicated to build a precise digital representation of heritage buildings.

Moreover, machine learning has aided experts in various fields by performing certain tasks automatically, saving human time and effort. With respect to the construction of architectural buildings, machine learning can assist in the creation of BIMs and HBIMs for buildings that do not possess such models. Instance segmentation, in particular, can help in the creation of digital models. Instance segmentation is a machine learning method where each object in the scan of a scene is identified as an instance separate from other instances. Where semantic segmentation outputs the different categories of objects present in a scene (e.g., door, window, chair, table), instance segmentation outputs the different instances within these categories (e.g., chair $\# 1$, chair \#2). Using the output of the instance segmentation, we can reduce the need for manual identification and segmentation of the different objects in a building. The entire process would be as follows: first, we would obtain a scan of a room in the building that needs to be modeled. Then, the different objects, i.e., heritage assets in the room would be identified with the aid of instance segmentation. The scan and identified instances would then be used to compose a BIM for the room. This process would be repeated for each room in the building to create the complete BIM. Finally, the different heritage assets could be tracked before and after the renovation without needing user supervision.

Instance segmentation methods based on machine learning need to be trained with adequate data. This involves manually preparing a large number of labeled scans of rooms. With LIDAR technology, we are able to scan point clouds that are very detailed but are not labeled. A lot of human intervention is required to prepare these scans to be used as training data. In order to reduce the human effort, semisupervised machine learning algorithms allow us to provide a balance between manual work and automatic segmentation. Specifically, by using a semi-supervised machine 
learning approach, we can use some labeled data to learn a segmentation model, but also let the user process data that was labeled incorrectly with minimal effort, saving time and effort in the overall process.

\subsection{Objective of the thesis}

The objective of this thesis is to introduce a method for instance segmentation of indoor environments represented by point clouds. As discussed above, instance segmentation can help in the creation of digital models of buildings. We focus on indoor environments because of our interest in heritage assets. The availability of datasets of indoor rooms is an added benefit that we can use to our advantage. We process point clouds since this is the main type of representation generated by 3D scanners.

After PointNet [38] introduced a way to process point clouds with deep learning, multiple applications started appearing with respect to classification or segmentation of point clouds. These methods, built on top of PointNet, extend the pipeline of deep learning and use it for instance segmentation. However, these methods $[9,21,23,24$, $36,37,48,50]$ require to be trained on labeled data related to the instances in a point cloud to predict instance labels, and they are mainly supervised approaches. One such method, a pioneer of instance segmentation, is the Similarity Group Proposal Network (SGPN) [50]. SGPN makes use of the features produced by PointNet and learns a similarity metric using instance labels. Another recent approach, called OccuSeg [21], voxelizes the point clouds and learns the space an instance occupies in terms of voxels to predict instance labels.

In contrast, our method does not require training data with instance labels, relying instead on clustering methods to discover the instances in the data. Specifically, a semantic labeling [29] is first used to separate the points into different categories. Then, for each category, our goal is to find a relationship between each point of an object to other points in the same object. We achieve that by clustering the points into different instances based on their semantic similarity. This is made possible by training PointNet to estimate point features that reveal the semantic similarity of points with a modified loss function. This can be seen as a form of metric learning. We call the version of PointNet that performs this type of learning PointSimNet, for brevity. Our approach then clusters the point features of the point clouds. We use the feature distance between points and the category that they belong to as two distinct 
measures to separate the instances. This saves time required to label the data as well as to train it.

In addition, learning the point features allows us to adapt our method to function as a semi-supervised approach to instance segmentation. This is an optional feature that we introduce to allow the users some authority over the improvement of the output, where the user can enter constraints to guide the result of the clustering. To the best of our knowledge, this process of semi-supervised instance segmentation is the first of its kind in the context of point cloud processing.

In terms of architectural data for heritage sites, the lack of labeled data has resulted in a lack of progress in the field of machine learning for heritage buildings. Thus, we demonstrate that we can use the information gathered from mining existing labeled data and apply it to perform instance segmentation of heritage buildings.

\subsection{Overview of the method}

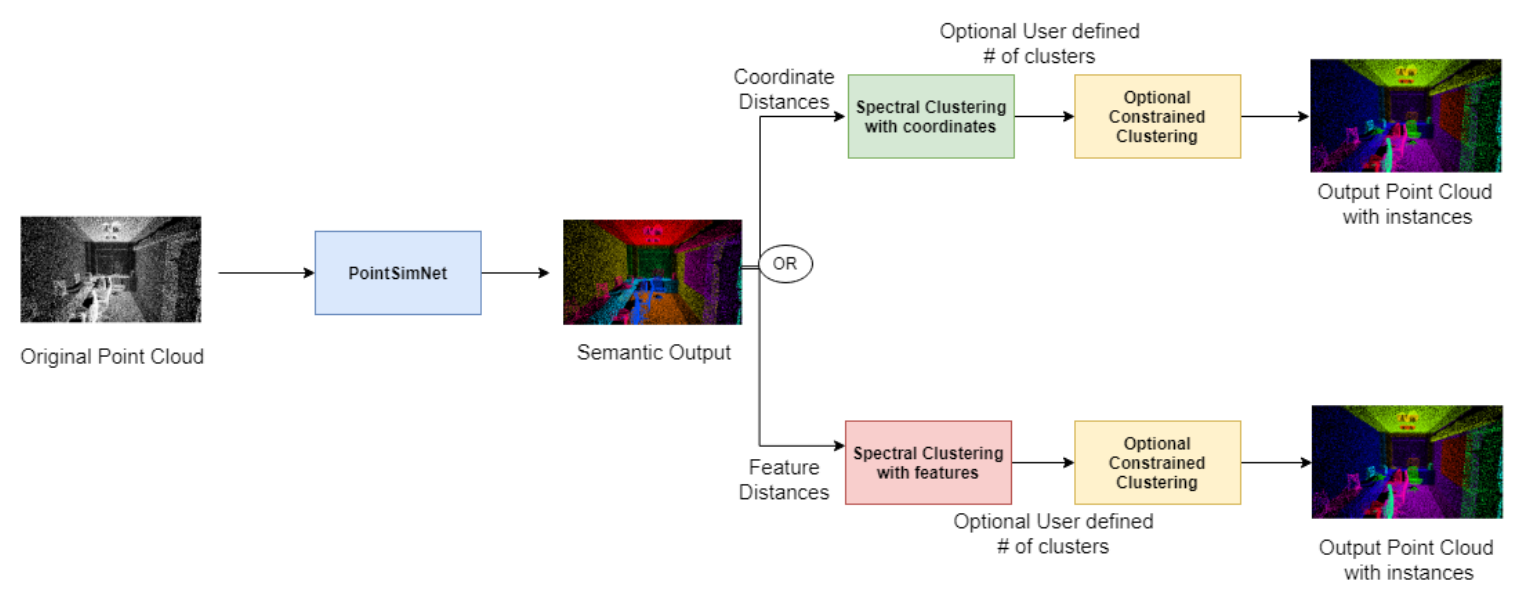

Figure 1.1: Overview of our method.

Given an input point cloud, our network PointSimNet predicts a feature vector and a semantic label for each point. The distances among feature vectors reflect the dissimilarity of points, in terms of the categories that the points belong to. PointSimNet is trained with a set of points clouds, where the points in each training cloud are labeled with a category label.

PointSimNet uses PointNet [38] as its base model. PointNet, being the pioneer neural network that directly processes point clouds, predicts point features (local and 
global) to perform classification and segmentation. We take advantage of these point features but modify how they are learned with a refined loss function inspired by SGPN [50]. The loss function trains the network in order to minimize the distance between points that belong to the same category and add a fixed distance or "gap" between points that belong to different categories. Using this approach, the point features that are then predicted by the network for unknown points clouds have similar values when points belong to the same category.

After predicting the point features and semantic labels for an input point cloud, we use the point features to cluster the input points into different categories. We also explore the use of the spatial coordinates of points for clustering. Specifically, we perform spectral clustering of the points. First, we build a distance matrix by subtracting the coordinates or features for each point from every other point. This distance matrix is then converted into an affinity matrix using a Gaussian heat kernel. The affinity matrix is then used for grouping the points with spectral clustering. In addition, the affinity matrix helps us to find the number of clusters using the eigengap heuristic.

Once we find the separate object instances in the form of clusters, the user has the option to manually refine the results with guided input. We perform this refinement with constrained clustering. The user identifies the number of clusters for each category as then adds cannot-link constraints to help improve the overall clustering and instance segmentation.

To the best of our knowledge, this novel semi-supervised approach to instance segmentation is the first of its kind in the processing point clouds with machine learning. We extract the information provided by the categories in the training data and do not require any extra information such as instance labels to perform the instance segmentation. We have devised three different ways of obtaining the instance labels depending on the usage scenario: the use of coordinates, the use of point features, and user intervention.

According to our knowledge, most of the previous instance segmentation methods based on machine learning require instance labels for training their segmentation networks $[9,21,23,24,36,37,48,50]$. Our approach learns mainly from category labels which are available in larger quantities in existing datasets, while it achieves results similar to methods trained with instance labels [50]. This is possible since we take advantage of similarity learning and clustering methods to discover the object 
instances.

\subsection{Summary of contributions}

This thesis provides the following contributions:

- We introduce a method for instance segmentation that does not require training data with instance labels. Our instance segmentation method is based on obtaining point features and semantic labels from PointNet [38] with a modified loss function, and then performing spectral clustering on the features.

- We also introduce a semi-supervised approach to perform instance segmentation based on clustering the features with constraints, where the user can refine the automatic segmentation results with minimal effort;

- The mean average precision of our instance segmentation results is 16 percentage points lower than the state-of-the-art method SGPN [50] and also lower than other concurrent methods [21,24], which require instance labels for training. However, when considering an alternative definition of the evaluation measure, we outperform SGPN by 20 percentage points without using instance labels.

\subsection{Organization of the thesis}

Chapter 2 discusses the different concepts of machine learning that are essential to this thesis. We start by defining machine learning and its two branches: supervised machine learning and unsupervised machine learning. We also define semi-supervised machine learning, a hybrid machine learning approach. Within the supervised machine learning section, we focus on discussing deep neural networks and the different components of such networks, such as convolutional layers, pooling, and activation functions. Within the unsupervised machine learning section, we explore the problem of clustering. In particular, we discuss the spectral clustering method that we use in our work. Then, in the semi-supervised clustering section, we explain the branches of constrained clustering and metric learning that are relevant to our work. Lastly, we discuss the existing literature on methods for processing point clouds, such as PointNet [38] and SGPN [50], and their applications. 
Chapter 3 presents our method for instance segmentation of point clouds. We extend the model developed by PointNet and introduce a new loss function for learning a deep network that predicts point features that reveal similarity between points. Then, we introduce the method we use for clustering points into instances. Finally, we introduce our user-guided method for performing constrained clustering of the points.

Chapter 4 discusses the results of our method. We analyze our semantic segmentation results as well as instance segmentation results. We also perform evaluation against one state-of-the-art method and show improved performance using our method. The improvement of our model is visualized in the qualitative results section and displayed in terms of statistics in the quantitative results section. We also show the improvements offered by our constrained clustering algorithm in this chapter as well as the time required to train and test our model.

Chapter 5 concludes our thesis and lists the limitations of our work. We also discuss ideas for future work. 


\section{Chapter 2}

\section{Background and literature review}

Since this thesis introduces a semi-supervised machine learning approach for processing point clouds, we will discuss machine learning approaches in Section 2.1, including supervised machine learning and deep neural networks in Section 2.1.1, unsupervised machine learning in Section 2.1.2, and semi-supervised machine learning in Section 2.1.3. We will also give an overview of point cloud data and general point cloud processing problems in Section 2.2, and machine learning algorithms applied to point clouds in Section 2.3.

\subsection{Machine Learning}

The development of machine learning in the field of artificial intelligence encompasses numerous advancements surrounding us. With huge amounts of data being collected everyday, machine learning algorithms are applied to process this data automatically. Example machine learning applications range from process automation and medical imaging to computer vision and face detection. More recently, the diversity of machine learning applications has increased tremendously, being used in products such as selfdriving cars, Amazon's recommendation system, and virtual personal assistants like Siri or Alexa. Machine learning algorithms discover trends in data and learn to induce conclusions from the data without direct user involvement. Machine learning problems can be categorized into supervised learning, unsupervised learning, and semisupervised learning. Reinforcement learning is another category of machine learning

but is beyond the scope of this thesis. We discuss these different types of machine learning as follows. 


\subsubsection{Supervised Machine Learning}

Supervised machine learning is one of the most popular machine learning techniques. In supervised learning, a method learns to map an input to an output according to a set of examples of inputs and their corresponding outputs. Thus, the input and output data come in pairs. Specifically, in classification problems, where the goal is to determine the label of a data sample, all the training data is labeled [19]. For example, to recognize whether an input image containing food is Mexican or Italian, supervised learning would require examples of pictures of food accompanied by labels. This data is called training data, as the method learns from this data. Moreover, test or testing data consists of data that the method is not aware of during training, which is used for evaluating the performance of the method. Splitting of data between training and testing ensures a way to evaluate the algorithm. Classification and regression are some of the problems solved by supervised learning algorithms. We further discuss classification and segmentation in Section 2.2.

\subsubsection{Deep Neural Networks}

Deep learning can be categorized into supervised and unsupervised methods. However, since in this thesis we use a supervised deep learning method, we focus on this category of methods in the following discussion. Deep learning, a subset of machine learning, makes use of multi-layer architectures of neurons to infer predictions for input data. This multi-layer architecture is called a Deep Neural Network (DNN). DNNs are inspired from biological organisms and, much like them, comprise of neurons connected to each other. In DNNs, artificial neurons are connected via a network of links called edges. Each input edge to a neuron has a weight assigned to it, which is adjusted according to the learning process. During the activation of the neuron, each weight is multiplied by its corresponding input. All these products are summed up, added to a bias, and passed through an activation function (discussed below), to produce the output of the neuron.

DNNs are comprised of multiple layers [35], where each layer is responsible for extracting different features of the data. The lower-level layers (closer to the input) extract the most simple or basic features of the data, while the higher-level layers extract more complex features. For example, in the medical imaging field, a deep network's first layer may be able to extract a nodule from a CT-scan, while the 
second layer may extract features of that nodule, and the output layer will predict the probability of the nodule being cancerous. The good performance of DNNs has resulted in hundreds of applications in medical imaging [25], computer vision, and graphics.

DNNs contain multiple layers including an input layer, an output layer, and one or more hidden layers. The layers can be of different types, such as convolutional layers followed by an activation function, pooling layers, or fully connected layers. We discuss some of these components in more detail as follows.

Convolutional layer. Convolution is the mathematical operation that produces a function based on the shape of one input function modified by the shape of a second input function (called a convolution kernel) [19]. These functions can be represented in a discretized manner as matrices. Given an input function represented as a matrix, the convolution kernel is slid over the input. The number of times the kernel slides over the input matrix depends on the stride (an input parameter). For each sliding step, each entry of the convolution kernel is multiplied with the corresponding entry of the input matrix, according to how the input and kernel are aligned. The resulting products are then summed up and this value is assigned to the entry in the output matrix corresponding to the position covered by the center of the kernel. The output matrix is called a feature map [19].

Pooling layer. A pooling layer often appears after the convolutional layer and is responsible for reducing the size of the feature map created by the convolutional layer. As the depth of the network increases, the number of feature maps would also increase, which could be computationally expensive to store and process. In this case, the pooling layer is responsible for down-sampling [41] the feature maps by reducing their spatial dimensions. Pooling methods are categorized into local pooling and global pooling. Local pooling acts on small clusters of input neurons, while global pooling works on all the input neurons [30]. The function applied on the values output by the neurons may be simply a maximum, resulting in Max Pooling, or a type of average, resulting in Average Pooling. The resulting matrix of a smaller size helps with reducing the complexity of the data being processed by the network. 
Fully-connected layer. As the name suggests, in a fully-connected layer, each input neuron of one layer is connected to every output neuron of the next layer. Fullyconnected layers are often connected to the output of convolutional and pooling layers to produce the final output values of the DNN.

Activation function. The output of a neuron is given by multiplying the values of the input edges of the neuron by their corresponding weights and then adding the bias value. As these are linear operations, with these neurons, the layers of the neural network can only learn linear transformations of the input [35]. To learn non-linear transformations, activation functions are applied to the output of selected neurons in order to learn more complex compositions of features, i.e., deep representations in multi-layer networks [1]. The activation functions can be placed after the hidden layers in neural networks and, in this case, they are responsible for converting linear mappings into non-linear mappings. If an activation functions is placed after the output layer, it can help in performing predictions.

Some common activation functions are the sigmoid function, tanh, softmax, and the rectified linear unit $(R e L U)$. PointNet uses ReLU as activation function. The output of ReLU ranges from 0 to infinity (no negative values). ReLU is computationally inexpensive compared to other functions [46].

Training DNNs. As mentioned above, supervised learning methods are often trained and evaluated by separating labeled data into training and testing data. The steps of training a neural network are as follows:

- Forward pass: The first training data sample is passed through the entire network. At the beginning of the learning process, the resulting output is inconsequential because the neuron weights are not meaningful yet.

- Loss function: The loss function compares the predicted output to the groundtruth output and provides an error value denoting the difference between the desired and predicted output. The loss function is a function such as the mean square error (MSE) or cross-entropy.

- Back Propagation (weight update): Using the gradient descent technique, the back propagation algorithm computes neuron weights that minimize the loss of the model [40]. Specifically, the back propagation method computes the gradient 
of the loss function, where the gradient points to the direction that increases the loss. Then, the method changes the weights in the opposite direction of the gradient, since the goal is to minimize the loss. The learning rate is a constant multiplied by the gradient to control the magnitude of the update. The learning rate is an empirical parameter. The higher the learning rate, the bigger the jump taken to update the weights and the faster the network will converge to a stable state. However, if the learning rate is too high, the steps taken will be too big and imprecise to achieve the learning goal.

All three steps above are part of one iteration of training. For one iteration, a batch is passed through the network, where a batch consists of a set of training data samples with a pre-defined size. Thus, the neuron weights are updated after considering one iteration [35]. To fully train the network, multiple epochs are used where each epoch signifies the passing of the entire dataset through the network.

Once the training process is finished, the model is evaluated using the test dataset. Ideally, the test dataset is kept separated from the training set.

In this thesis, the two main supervised machine learning networks used are PointNet [38] and SGPN [50]. These networks are based on convolutional neural networks and are discussed in Section 2.2.

\subsubsection{Unsupervised Machine Learning}

Unsupervised machine learning is used when the goal is to understand the properties of the structure of the data [19]. It is also called self-organization and encompasses modeling of probability densities of inputs [7]. Two well-known categories of unsupervised learning are dimensionality reduction [35] and clustering [45].

\subsubsection{Clustering}

Clustering methods group data points based on their similarity. The data points in a group are called a cluster. A point in a cluster is more similar to the points in the same cluster compared to another cluster, where the similarity measure is typically application-dependent. The measure of similarity could be, for example, the distance between points, density of points, or connectivity. Moreover, the resulting number of clusters varies according to the algorithm used [15]. Some common types of clustering 
methods are Connectivity-based, Density-based and Centroid-based clustering. We use a spectral clustering method which is described below.

Spectral Clustering. Spectral clustering treats the problem of clustering like a graph partitioning problem. It considers the data points as nodes in a graph and groups the nodes based on the connectivity of the nodes in the graph, where the connectivity between nodes is derived from their similarity. Spectral clustering outperforms traditional methods like connectivity-based and centroid-based clustering algorithms on difficult inputs [47]. For example, given a swiss roll dataset [44], spectral clustering can provide a more meaningful clustering result because it can group points along the roll. Other clustering methods such as k-means clustering group points across different layers of the swiss roll. In the case of k-means, this happens since the method performs the grouping based on the Euclidean distance from the centers of the clusters.

To cluster the data points by partitioning a graph, spectral clustering takes as input a matrix encoding the similarity between all pairs of data points, called a similarity matrix or affinity matrix. This matrix can be considered as a weighted adjacency matrix of a fully-connected graph, where each data point is a node in the graph, and the weight of the edge between two points is the value of similarity between the two points. Then, we derive a Laplacian matrix from the graph, and compute its eigenvalues and eigenvectors to find the expected number of clusters and perform the clustering itself. The eigenvectors provide an embedding of the data points onto a space where the clustering problem can be solved more easily. We use spectral clustering for our method, given that spectral clustering is able to provide more meaningful solutions than other clustering methods for challenging inputs [47]. We provide more details on spectral clustering when describing our method in Chapter 3.

\subsubsection{Semi-Supervised Machine Learning}

There are many advantages to learning with labeled data, such as the direct specification of the learning goal and the possibility of evaluating the performance of the model. However, due to a limited availability of labeled data, human input is often needed to create annotations of unlabeled data to train supervised learning methods. This can be costly and time-consuming. To get the best of both approaches, 
semi-supervised learning makes use of both labeled and unlabeled data. It lies between supervised and unsupervised learning and builds its methods on top of these two learning frameworks [53], to solve tasks such as classification or clustering. In a "standard" semi-supervised setting [53], the input data can be divided into two portions: one for which labels are available and one which is unlabeled. Then, with semi-supervised machine learning approaches, we learn from both the labeled and the unlabeled data [54]. Metric learning and constrained clustering are two important semi-supervised methods, which we use in our work and explain in more detail below.

Constrained Clustering. Constrained clustering implies that the clustering is computed with constraints directly provided by a user. Typically, we can specify must-link and cannot-link constraints. Must-link constraints define data points that should belong to the same cluster. Cannot-link constraints define data points that should not belong to the same cluster. Specifically, given a similarity matrix that represents how similar all points are amongst each other, a must-link constraint between two points can be implemented by increasing the similarity value between the two points in the similarity matrix [5]. It is used when we have some pre-existing knowledge about the desired partition of the dataset into different clusters [43]. Constrained clustering has been incorporated into different clustering algorithms, such as K-means, spectral clustering, hierarchical, and density-based clustering algorithms.

Constrained spectral clustering can be achieved by manipulating the Laplacian or affinity matrix [27]. In this category of methods, the entries of the Laplacian or affinity matrices (that represent the similarity between points) are modified to incorporate the constraints. For example, in the method firstly introduced by Kamwar and Klien [26], if two data points $i$ and $j$ should be linked together, then the affinity matrix value $A_{i, j}$ is set to 1 . Similarly, if $i$ and $j$ should not be linked together, then the affinity value $A_{i, j}$ is set to 0 .

In our method, we use constrained clustering based on modifying the input similarity matrix, which allows us to easily incorporate user-given constraints for clustering.

Metric Learning. Metric learning refers to learning a distance function specific to a problem, according to training data [8]. The learning is also performed with constraints that specify the required distance between points. The constraints can be positive, negative, or relative. Positive constraints specify points that should be linked together and are defined as: 


$$
S=\left\{\left(x_{i}, x_{j}\right): x_{i} \text { and } x_{j} \text { should be similar }\right\} .
$$

Negative constraints specify points that can not be linked together:

$$
D=\left\{\left(x_{i}, x_{j}\right): x_{i} \text { and } x_{j} \text { should be dissimilar }\right\} .
$$

Relative constraints constrain triplets:

$$
R=\left\{\left(x_{i}, x_{j}, x k\right): x_{i} \text { should be more similar to } x_{j} \text { than to } x_{k}\right\} .
$$

The metric learning algorithm takes in these constraints and learns a model. The model can then predict an output similarity matrix for an input set of points, such that the similarity among the points best fits the constraints.

Metric learning has multiple applications such as image retrieval [3] and face recognition [11]. The SGPN method for instance segmentation [50] takes this metric learning approach and builds up a similarity matrix based on the likelihood of two points belonging to the same instance. We discuss this method in Section 2.3.

\subsection{Point Clouds}

Point clouds are composed of data points set in 3D space. Point clouds can be created with photogrammetry, where photos or scans are taken at various angles of the desired location or object and then reconstructed into a 3D model. Photos or scans collected at different viewpoints are then stitched together to form a scene in a process called registration. Registration or point matching aligns point sets using transformations. Iterative closest point (ICP) [14] and robust point matching (RPM) [17] are examples of point registration algorithms.

Points are normally described by $3 \mathrm{D}$ coordinates $(x, y, z)$ and may have additional attributes such as RGB color or normal. Point clouds are often converted to a 3D surface like a mesh or a CAD model for visualization through a process called surface reconstruction. Surface reconstruction techniques include the marching cubes algorithm [10]. Point clouds are used in the creation of models of existing buildings or objects, quality inspection of surfaces, and various visualization and animation applications.

Processing point clouds is a challenging problem [2]. This is due to the lack of 
connectivity information from the original surface and scanning-related problems that may result in noisy or incomplete data depending on the technology used. In addition, multiple point clouds representing an object can be considered non-Euclidean geometric data, which means that they do not have a structure for global parameterization or a common system of coordinates. However, if the point clouds are processed locally, they may be categorized as Euclidean data.

Due to their irregular format without a parameterized domain, most of the traditional point cloud processing methods first convert the input point clouds to other forms of data, such as voxelized shapes [51] or 2D images [22], which results in voluminous data and produces artifacts. Point cloud feature learning algorithms have been classified into point-based and tree-based [31]. Point-based models directly take

a point cloud as input, whereas tree-based methods first convert the point cloud into a tree data structure.

Within deep learning models for point cloud processing, PointNet [38] is the first approach to directly take a point cloud as input. PointNet can be used for classification or segmentation, and thus outputs labels for either each point in the point cloud or the entire input. We further explain this method in Section 2.3.

\subsubsection{Point Cloud Problems and Solution methods}

There are several analysis problems related to point clouds, including object classification, segmentation, and instance segmentation. The differences between these problems are discussed as follows:

Point Cloud Classification. Classification assigns a label to a group of points. For example, given a point cloud, a classification problem may consist of labeling a point cloud to identify a scan of a chair or a table. Classification is the most common problem in machine learning. Methods for solving classification problems can be divided into supervised and unsupervised approaches. Supervised methods learn to perform the labeling of the input based on training data, whereas unsupervised methods classify based only on a small set of provided parameters, such as the number of clusters in k-means clustering.

Point Cloud Segmentation. Segmentation consists in partitioning an input point cloud into subsets, where a group of points belonging to a subset is called a segment. 
Segmentation can refer either to scene segmentation or part segmentation, depending on whether the point cloud represents an entire scene which is partitioned into objects, or an object which is partitioned into parts. The segments may also be assigned a label by the segmentation method. For example, given a point cloud of an office scene, a segmentation method may divide the points into groups labeled as "chair" or "table". All the points belonging to a segment often share a common characteristic, such as a geometric or radiometric property [20].

Point Cloud Instance Segmentation. Instance segmentation takes semantic segmentation a level further. In instance segmentation, instead of only identifying the different categories that points belong to, the problem also requires to identify each individual object that is part of the category. For example, given an office scene, semantic segmentation would identify points labeled as "chair" and "table", while instance segmentation would separate all the points labeled as "chair" into different instances (individual objects). The difference between semantic and instance segmentation is illustrated in Figure 2.1. The input point cloud is shown on the left. The middle image shows the point cloud labeled with semantic labels, where each color represents a semantic category (for example, all the chairs have red color). The right-most image shows the point cloud labeled with instance labels, where each color represents the separate instances (for example, one chair has an orange color while the other chairs have colors such as blue and green). While instance segmentation has been receiving a lot of attention in the past few years, a deep learning-based method for point cloud instance segmentation was first introduced by Wang et al. [50] (the SGPN method).

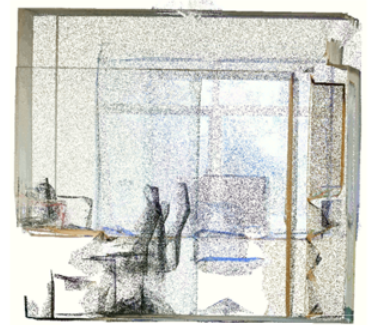

Input Point Cloud

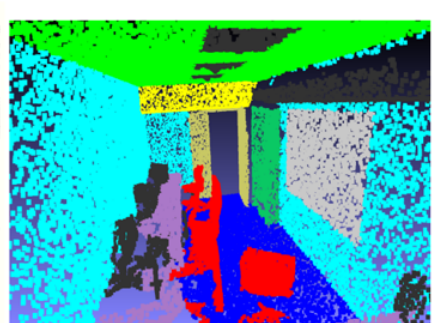

Semantic Segmentation

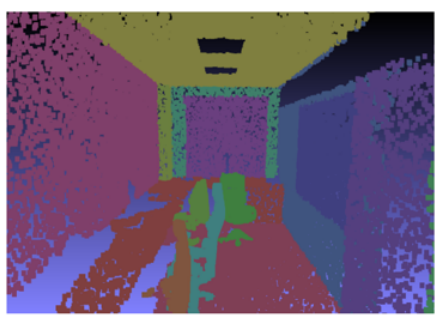

Instance Segmentation

Figure 2.1: Difference between semantic segmentation and instance segmentation. 


\subsection{Point Cloud Processing with Machine Learn- ing}

In this section, we first discuss the pioneer method, PointNet, for processing point clouds with deep networks. After that, we describe the different methods that process point clouds that are based on PointNet and other recent methods that perform instance segmentation with neural networks.

PointNet. Qi et al. introduced PointNet [38], the first deep neural network that can process point clouds without the need of converting them to a different data format. PointNet can be used to perform object classification, part segmentation, and scene segmentation. The PointNet model is invariant to permutations of the input, which means that the points in the input point cloud can be given in any order. PointNet captures meaningful neighborhoods between points and is also invariant to transformations like rotation or translation.

The PointNet architecture is composed of a symmetric function (the max-pooling layer described below), combining local and global features, and an alignment network called T-Net, as illustrated in Figure 2.2.

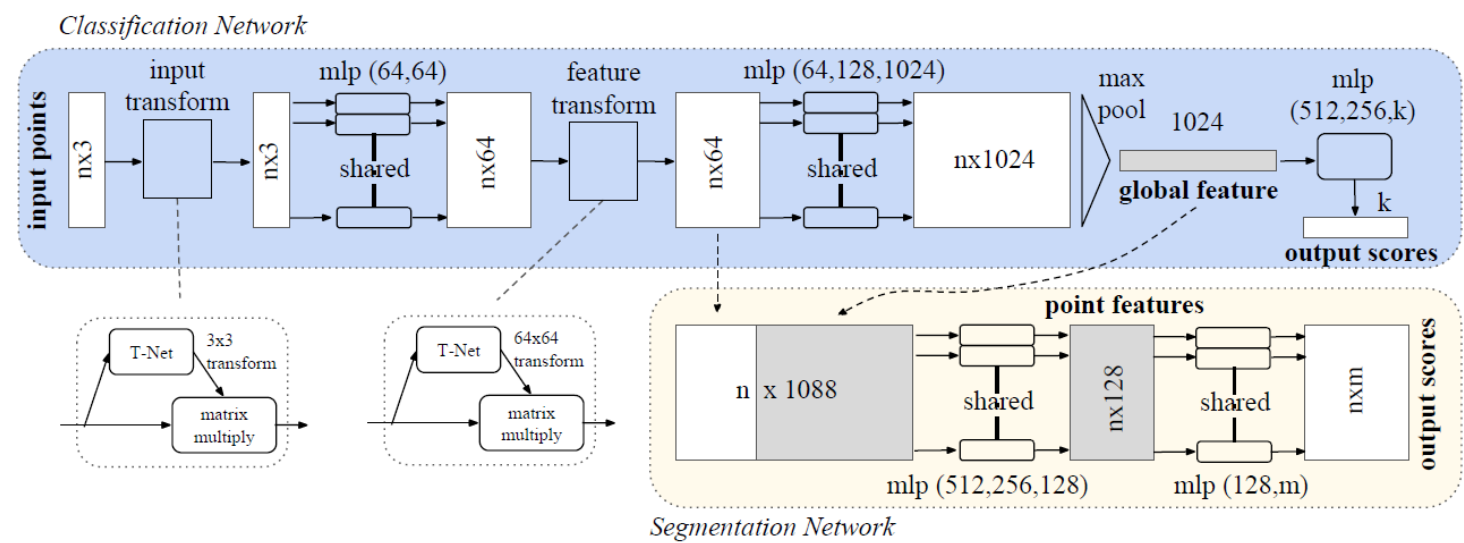

Figure 2.2: PointNet architecture, where MLP denotes "multi-layer perceptron network". Image source: Qi et al. [38].

The first important part of the model, the symmetric function, ensures that the result is invariant to permutations of the input points, via the following property [38]:

$$
f\left(\left\{x_{1}, \ldots, x_{n}\right\}\right) \approx g\left(h\left(x_{1}\right), \ldots, h\left(x_{n}\right)\right),
$$


where $h$ represents the result of a function calculated by a multi-layer perceptron network (MLP) and $g$ is approximated by composing a max-pooling function and a single variable function to capture the different properties of the input, which ensures that $g$ calculated on different permutations of the input provides the same output. The result of the function $f$ is a set of aggregated high-dimensional features learned by a MLP.

The next vital part of the PointNet architecture is the aggregation of local and global features. Because the symmetric function only provides the global features of the points, segmentation cannot be performed until local information is acquired. As seen in Figure 2.2, a Segmentation Network takes the global features collected by the max-pooling function and concatenates them with point features, creating per-point features that are both local and global in nature.

Lastly, the alignment network ensures the model's ability to identify objects regardless of their transformation. It is employed at two stages in the network as indicated by the alignment network T-Net applied to the input and at the feature transform. The T-Nets are called "mini-PointNets" [38] and are composed of shared MLP networks (which consist of convolutional, ReLU, and batch normalization operations), fully-connected, and max-pooling layers. The semantic learning process is explained in Section 3.3. The transformation of the input point cloud outputs a $3 \times 3$ matrix, whereas the transformation of the feature transform gives a $64 \times 64$ matrix, since the input points are 3D and the features have 64 dimensions.

The overall flow of the network is as follows:

- The network is given $n$ points of a point cloud as input;

- Input points are transformed by the input transformation network;

- The points are then embedded by a multi layer perception onto a $n \times 64$ dimensional embedding space;

- The feature transformation is applied;

- Then, the points are embedded onto a $n \times 1024$ dimensional space;

- Max-pooling aggregates all points in the high dimensional embedding space and outputs them as global features;

- The classification network outputs $k$ scores for $k$ classes; 
- The local embedding plus the global feature vectors are concatenated into features that can be used for per-point classification (segmentation), resulting in $m$ scores for each point.

PointNet++. Qi et al. [39] introduced a hierarchical neural network that addresses the limitation of PointNet not capturing the local structure of point clouds. Basically, PointNet learns the local features for each point using a MLP which does not enable the network to learn the relationship between the points. Therefore, it does not handle local feature extraction [31]. To solve this problem, PointNet++ partitions the input point set and applies PointNet recursively on each partition. It also handles the problem of point sets which are sampled with varying densities by introducing a technique to learn features using different scales. PointNet++ shows considerable improvement in segmentation over the original PointNet where local features are combined with global features. However, we base our model on PointNet rather than PointNet++ since one of our design goals is to avoid partitioning the input into blocks, which cause visual artifacts in the output (see Figure 4.4).

A number of subsequent methods have used PointNet or PointNet++ as a base network and modified it to create models to suit specific needs. Some of these models, which also address the instance segmentation problem, are listed below:

Frustum PointNets. Frustum PointNet [37] detects objects at the instance level in RGB-D images using 2D object detectors alongside 3D deep learning. A Convolutional Neural Network (CNN) is used to generate 2D object regions from the RGB-D input data. Each 2D region is then transformed into a 3D viewing frustum which provides a point cloud. The output is given to a deep network that outputs bounding boxes around the objects detected in the point cloud. Thus, this network is relevant for single RGB-D images that have not yet been registered into a point cloud.

SGPN. Wang et al. introduced SGPN [50], which is the pioneer algorithm to perform instance segmentation on point clouds with a deep neural network. SGPN is built on PointNet, as illustrated in Figure 2.3. 


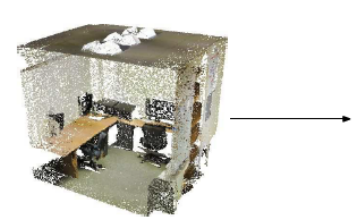

Input Points: $\mathrm{Np} \times \mathrm{Nf}$

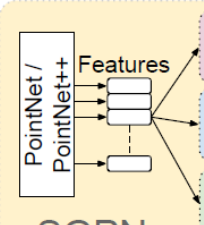

SGPN
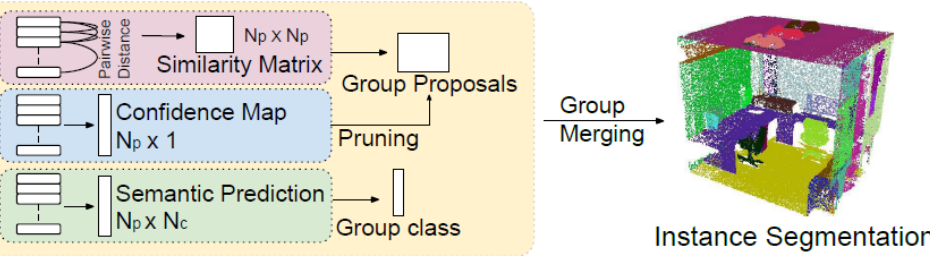

Figure 2.3: SGPN architecture. Image source: Wang et al. [50].

SGPN modifies point features provided by PointNet to reflect the similarity between points in terms of their semantic and instance labels. Then, the method builds a similarity matrix $N_{p} \times N_{f}$ and a confidence map for instance segmentation, to provide group proposals that indicate which points belong in which group. The similarity matrix defines the pairwise distances between the feature vectors of the points. The confidence map indicates the confidence of each group candidate becoming an object instance. Both of these matrices are then used to form group proposals to indicate which points should form an instance. The loss function for training the similarity model is defined below:

$$
L_{\text {sim }}=\sum_{i}^{N_{p}} \sum_{j}^{N_{p}} l(i, j),
$$

with

$$
l(i, j)= \begin{cases}\left\|F_{S I M_{i}}-F_{S I M_{j}}\right\|_{2}, & \text { if } C_{i j}=1, \\ \alpha \max \left(0, K_{1}\left\|F_{S I M_{i}}-F_{S I M_{j}}\right\|_{2}\right), & \text { if } C_{i j}=2, \\ \max \left(0, K_{2}-\left\|F_{S I M_{i}}-F_{S I M_{j}}\right\|_{2}\right), & \text { if } C_{i j}=3,\end{cases}
$$

where $C_{i j}$ indicates the similarity class that $i$ and $j$ belong to: same object instance $\left(C_{i j}=1\right)$, same semantic class but different object instance $\left(C_{i j}=2\right)$, and different semantic class $\left(C_{i j}=3\right) . F_{S I M}$ denotes the similarity features. $K_{1}, K_{2}$, and $\alpha$ are constants where $\alpha>1, K_{2}>K_{1}$.

Due to the above loss, points that are part of the same instance and class are constrained to be close together in the feature space, while points that are neither of the same class nor belong to the same instance are constrained to be the further apart. The loss used in our method is inspired by this loss, as we discuss in Chapter 3.

Three other methods, introduced in 2019, use PointNet or other types of convolutional networks to perform instance segmentation on point clouds. All of these methods require instance labels to train their networks. The details are given below: 
3D-BoNet. Built on top of PointNet [38] and PointNet++ [39], 3D-BoNet [9] produces 3D bounding boxes and predicts per-point masks. Local features of each point combined with global features from the entire point cloud are extracted from the backbone network (PointNet and PointNet++). Bounding box prediction is achieved using global features. Their use of instance labels is unique in a way that they find the best match between a predicted bounding box and a ground-truth bounding box, minimizing the difference between the boxes to obtain an accurate bounding box for the instances. The results of the bounding box combined with global features and local point features are then used to predict per-point masks for instance segmentation.

ASIS. A point cloud is encoded into a feature matrix using layers of PointNet [38] and PointNet ++ [39]. ASIS [48] then in parallel decodes the feature matrix into a semantic feature matrix and an instance feature matrix. To obtain instances for points, ASIS performs "Semantic-aware Instance Segmentation", where the semantic feature matrix is adapted into an instance feature matrix to produce semantic-aware instance features. They also use the instance labels to aid in semantic segmentation, calling this process "Instance-fused Semantic Segmentation". Once the final instance embeddings are generated, mean-shift clustering is applied on the instance feature matrix to label instances.

JSIS3D. Pham et al. [36] introduce a multi-task point-wise network (MT-PNet), based on PointNet, to perform the tasks of semantic and instance segmentation simultaneously. MT-PNet uses overlapping windows to scan the entire point cloud. The network encourages the points that belong to the same instance closer together and those that do not belong to the same instance to be pulled apart. They use instance labels to find the centroid of each instance and pull the points to that centroid if they belong in the same instance. Finally, they put the instance embeddings through Multi-Value Conditional Random Fields to obtain instance labels.

A few additional methods take point clouds as input but voxelize them to perform instance segmentation. These are the most recent methods (from 2020), developed concurrently with our work, and have achieved the top results in the field. They are discussed as follows: 
OccuSeg. OccuSeg [21], focuses on the occupancy of instances in terms of number of voxels. The input to OccuSeg is a voxelized 3D point cloud. OccuSeg extracts features from the volume using a CNN modeled after a network called U-Net [42]. These features are then used to learn a spatial embedding, feature embedding, and occupancy map. After obtaining the embeddings and occupancy map, the method forms a graph utilizing the feature embedding and occupancy map and performs clustering on the graph. The clustering method used is a graph-based segmentation algorithm. The result of the clustering is the final set of instances.

PointGroup. PointGroup [24] performs instance segmentation by exploring the space between object instances. Using U-Net [42] as a backbone network, the method extracts semantic labels and point features by voxelizing the input points. Simultaneously, the method also brings points from the same instance closer together by shifting them towards the centroid of the instance. These points are called offset points. Next, the method clusters both the original and offset points by progressively grouping neighboring points with the same label. This method is called "Dual-Set Point Grouping" since it makes use of the original and offset points. After obtaining the clusters, a network called ScoreNet [24] is used to attain instance labels for each point.

The instance segmentation methods for point clouds described above use supervised machine learning to learn from training data that contains instance labels. The two concurrent methods OccuSeg [21] and PointGroup [24] use voxelized point clouds to perform instance segmentation. In contrast, our approach to instance segmentation takes in input point clouds directly and makes use of semantic labels to output instance information. 


\section{Chapter 3}

\section{Instance Segmentation method}

Our method for solving the instance segmentation problem has the following steps, illustrated in Figure 3.1. First, we learn a similarity measure of points with a modified version of the PointNet model. We call this model PointSimNet. More specifically, given an input point cloud, the output of PointSimNet is a set of point features that can be used to establish the similarity among the input points. In addition, similarly to PointNet, PointSimNet also outputs a semantic segmentation of the points, assigning a semantic label to each point. Next, we partition the input points according to the semantic classes, and create a similarity matrix for each class by computing the pairwise feature similarity of all points. Then, we cluster the points of each class according to the similarity matrix. Finally, we improve the clustering according to user input with a constrained clustering method, which can be based either on the point coordinates or the similarity matrix. We explain each of these steps as follows.

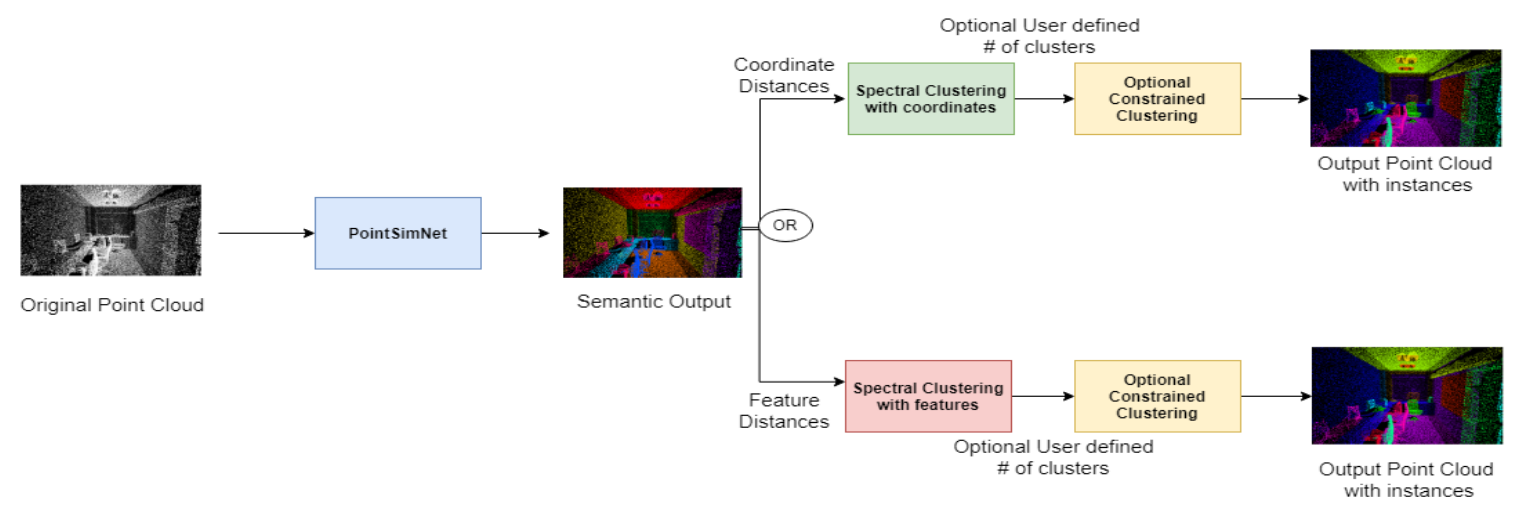

Figure 3.1: Overview of our method for instance segmentation. 


\subsection{Data assumptions}

In this section, we describe the requirements of our model on the input data, independent of the source of data. The details of the datasets that we use in our experiments are given in Chapter 4 .

As a supervised machine learning algorithm requires a training dataset that it can learn from, our model assumes the availability of a dataset of point clouds resulting from scanning individual rooms. The points in each point cloud possess attributes such as 3D coordinates, RGB values, and a label describing the semantic category that the point belongs to, e.g., wall, floor, chair, and so on.

The requirements for the test dataset are not as strict. For evaluating our method, the test dataset also has to contain labels with the semantic categories and instances of the points. However, as our algorithm is designed to be used in heritage projects, specifically BIM modeling, a test room can also just contain points with 3D coordinates and RGB values.

Our model then learns from the training dataset and assigns semantic labels to the test dataset. Using the semantic labels and point features produced by our model, we perform instance segmentation.

\subsection{Learning of Similarity measure}

PointNet is the pioneer neural network that is able to directly process an input point cloud. As discussed in Chapter 2, PointNet learns to predict a feature vector for each point in the point cloud, which is composed of a global set of features related to the entire point cloud, and a local set of features related to the point in question. These feature vectors describe the attributes of the points and help in the segmentation and classification of the input point cloud. For segmentation tasks, the local and global features are used to group the points into different categories. However, these features do not directly reflect the similarity among points and thus cannot be used to estimate a similarity matrix for clustering. Inspired by the SGPN [50] method, we train the network to predict the point features using a loss function that imposes similarity constraints on the features. However, SGPN requires labels for individual instances in the training data. Since we are working with data without instance labels, our method learns only from the semantic labels of the training data. 
More specifically, we train the network to predict point features for an input point cloud, where the distances among the features capture the dissimilarity of points. Thus, implicitly, we are learning a similarity measure that can be applied to any input point cloud. The feature prediction is learned with our loss function $L_{\text {sim }}$ given as follows:

$$
\begin{gathered}
\qquad L_{\mathrm{sim}}=\sum_{i}^{N_{p}} \sum_{j}^{N_{p}} l(i, j), \\
\text { with } l(i, j)= \begin{cases}\left\|F_{i}-F_{j}\right\|_{2}, & \text { if } C_{i j}=1, \\
\max \left(0, K-\left\|F_{i}-F_{j}\right\|_{2}\right), & \text { if } C_{i j}=2,\end{cases}
\end{gathered}
$$

where $N_{p}$ is the number of points in the training instance point cloud, $C_{i j}$ indicates the semantic class that $i$ and $j$ belong to (same semantic class when $C_{i j}=1$, or different semantic class when $\left.C_{i j}=2\right), F_{i}$ indicates the feature vector learned for point $i$, and $K$ is an arbitrary constant. This loss function is added for all the point clouds used during the training.

When minimizing the loss above, the distance between point features belonging to the same category is constrained to be as small as possible, while the distance between point features belonging to different categories is constrained to be $K$. The value of $K$ can be any desired constant other than zero, which controls the distance or "gap" between points of the same and different class in the embedding space. In our work, we set $K=1$. Thus, in this manner, an embedding space is learned where the distance among points reflects whether they belong to the same category or not.

We learn the point features $F_{i}$ with a PointNet neural network using the loss function above. We call this resulting approach the PointSimNet model, for brevity.

Once the network has been trained, we can apply it to any input point cloud to obtain an $N_{p} \times N_{f}$ feature matrix, where each row of the matrix are the embedding coordinates of a specific point. $N_{f}$ is the length of each feature vector, which is selected experimentally when designing the network. In our work, we set $N_{f}=128$. 


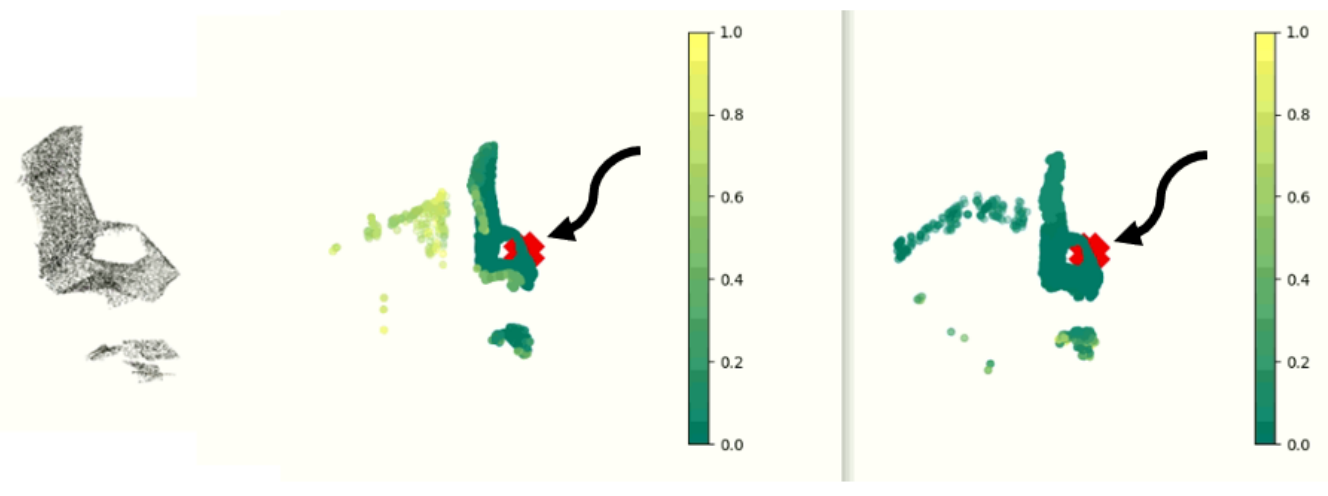

Figure 3.2: Comparison of the similarity of point features of a chair predicted by PointSimNet (center) and PointNet (right). The input point cloud is shown on the left.
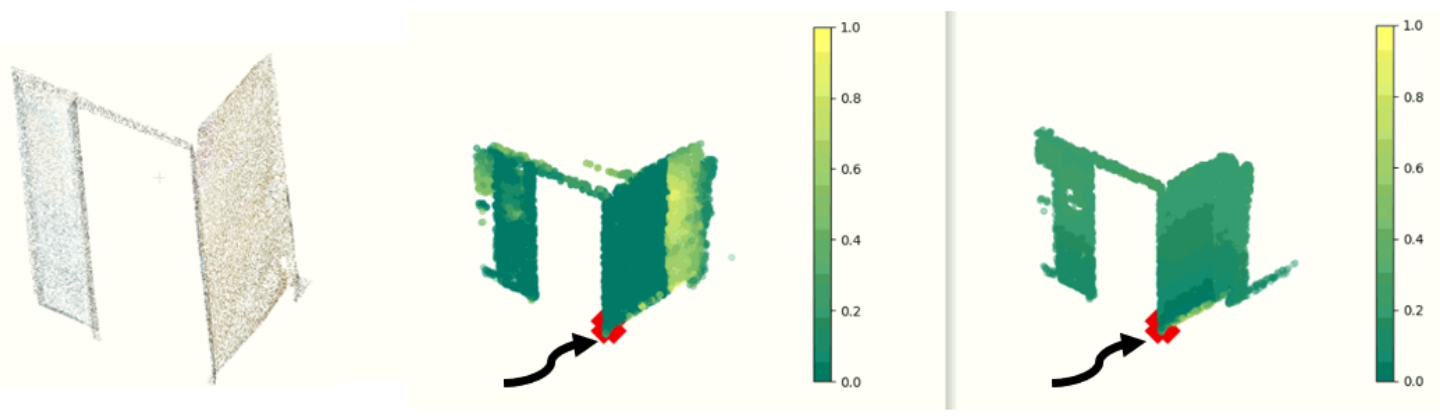

Figure 3.3: Comparison of the similarity of point features of a door predicted by PointSimNet (center) and PointNet (right). The input point cloud is shown on the left.

To demonstrate the benefit of learning the point similarity with PointSimNet, we show two visual examples of the learned similarities in Figures 3.2 and 3.3. In these examples, we pick a point on the given point cloud (the red area shown by the arrows in the figures) and calculate the distance from the features of the selected point to the features of every other point. The scale of the colormap shows the distance between all the points to the point selected which have been normalized between 0 and 1 by dividing all the values by the maximum similarity. The darker colors closer to green represent similar features (i.e., less distance between features and values closer to 0) while the lighter colors closer to yellow represent different features (i.e., more distance between features and values closer to 1 ). We then compare the features 
learned by PointSimNet to those learned by the original PointNet. We see how the features learned by PointSimNet provide a more meaningful distance among points. For example, the red point on the chair arm in Figure 3.2 is quite similar to other points on the chair, but dissimilar to the outlier points floating behind the chair. The same does not happen for PointNet, where the outliers are also similar. Thus, when semantic segmentation results are not perfect, we can use the learned features to better distinguish the points of one category from the points that do not belong in that category. Then, we can use the similarities to guide the instance segmentation.

\subsection{Learning of Semantic labels}

In addition to learning point similarity, PointSimNet also learns to perform semantic labeling of points, which consists of assigning a class label to each point in the input point cloud. Our semantic learning is based on PointNet with a modified loss function. PointNet uses global and local features of all points in the point cloud to perform semantic segmentation. As described in Chapter 2, the following properties are important for the model: the network needs to be invariant to the permutation of the input, meaningful information regarding the neighborhood of the points should be extracted, and the network should be invariant to any transformation of the point cloud.

The PointNet model proceeds as follows. Given $n$ number of points in three dimensions, $(x, y, z)$, a geometric transformation is first applied to the points using TNet. This process is illustrated in Figure 3.4. The transformation is performed with a mini-PointNet network comprising of a multi-layer perceptron network (MLP), which embeds the points onto a 1,024-dimensional space. The embedding of the points, after the application of max pooling, is used as a vector of global features to represent the point cloud. These features are then further embedded onto a 256-dimensional space using fully-connected layers. The features from this process are then combined with weights and biases that result in a matrix transformation. 


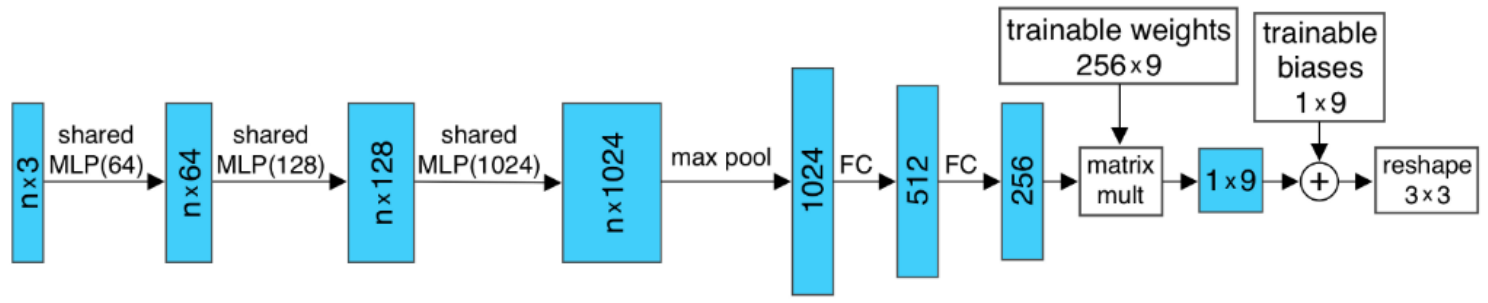

Figure 3.4: Overview of the T-Net Process. Image source of Luis Gonzales [18].

After transforming the input point cloud, a MLP is used to embed the points onto a higher-dimensional embedding space of 64 dimensions. Feature transformation, similar to the input transformation, takes place after that. The difference is the dimension of trainable weights, which increases from $256 \times 9$ in the input transformation to $256 \times 4,096$ in the feature transformation. The biases also increase from $1 \times 9$ to $1 \times 4,096$. Due to the number of trainable parameters in the feature transformation, a regularization term is added to the loss to remove the potential of over-fitting and instability during training. The points after feature transformation in 64-dimensional space are termed as local features.

After the feature transformation, a MLP is used to embed the points onto 1,024 dimensions. Then, max pooling is used to extract global features from the embedding. For segmentation, local and global features are combined to provide a feature vector of 1,088 dimensions. Then, a MLP is used again to embed the points onto a lowerdimensional space of 128 dimensions, and finally to a vector of scores with the number of categories present $(m)$. The result of the segmentation network is an $n \times m$ vector with the class scores for all the points in the point cloud. The architecture that carries out this overall process is illustrated in Figure 3.5. 


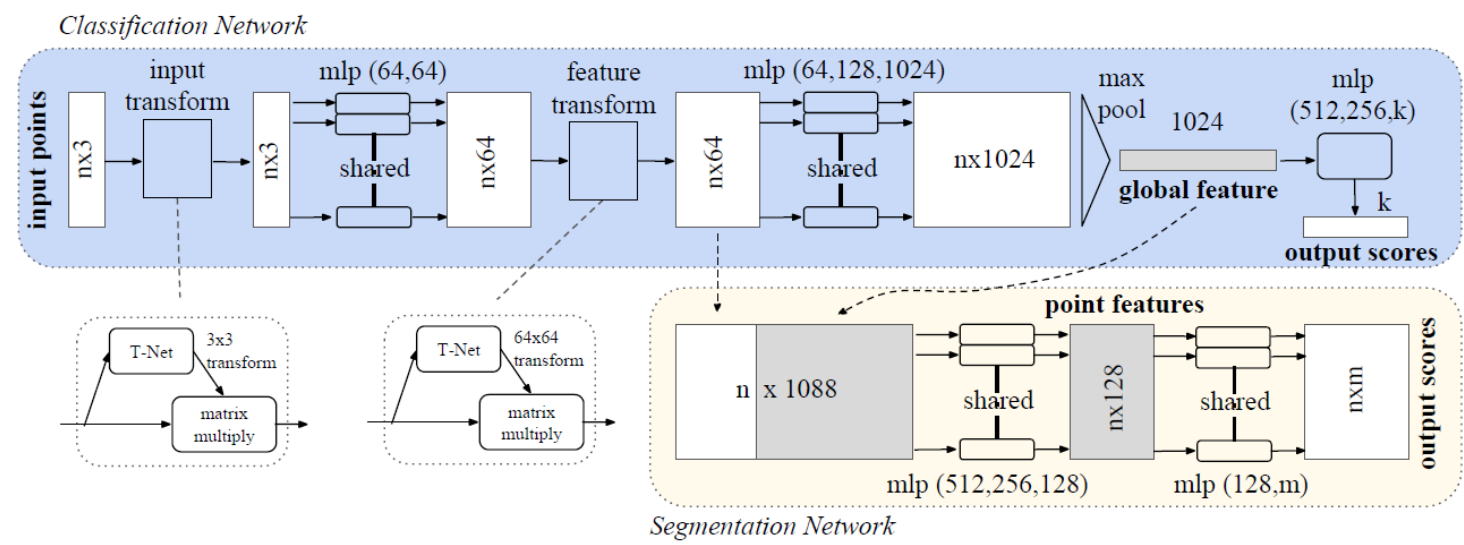

Figure 3.5: PointNet architecture. Image source: Qi et al. [38].

\subsection{Composing a Similarity matrix}

After filtering out the points belonging to a specific category, we create a similarity matrix for all the points in the category. This similarity matrix can then be used to cluster the points into different instances of the same category. The category labels of each point cloud are also predicted by PointSimNet as described above.

To create the similarity matrix, we have two point attributes that we can use to establish point similarities: using the point features predicted by PointSimNet or the original point coordinates.

When using the features predicted by PointSimNet, we create a distance matrix by calculating the $L_{2}$ norm between the feature vectors of each pair of points:

$$
D(p, q)=\left\|F_{p}-F_{q}\right\|_{2},
$$

where $p$ and $q$ are points belonging to the same category and $F_{p}$ and $F_{q}$ are the feature vectors of $p$ and $q$, respectively.

When using point coordinates, we calculate the Euclidean distance between each pair of points in the category using the equation below (which is essentially also a $L_{2}$ norm between point coordinates):

$$
D(p, q)=\sqrt{\left(p_{x}-q_{x}\right)^{2}+\left(p_{y}-q_{y}\right)^{2}+\left(p_{z}-q_{z}\right)^{2}},
$$

where $p$ and $p$ are points belonging to the same category, and $\left[p_{x}, p_{y}, p_{z}\right]$ and $\left[q_{x}, q_{y}, q_{z}\right]$ 
are the 3D coordinates of $p$ and $q$, respectively.

Then, to create the pairwise similarity matrix used for clustering, we convert the distance matrix into an affinity/similarity matrix using a Gaussian kernel:

$$
S(p, q)=e^{\frac{-(D(p, q))^{2}}{2 \delta^{2}}}, \quad \forall p, q
$$

where $D$ is the given distance matrix (computed with features or coordinates), $\delta$ indicates the width of the Gaussian kernel, and $S$ is the resultant similarity matrix.

The value of $\delta$ is dependent on the data. Common values for $\delta$ include the mean of the data or the maximum of the data, multiplied by some constant, since these would scale all the distance values according to the average or maximum distance in the point cloud. In Chapter 4, we investigate experimentally an appropriate value for the constant $\delta$.

We then cluster the points according to $S$ with the spectral clustering method, described as follows.

\subsection{Clustering the Similarity matrix}

The clustering process for instance segmentation is divided into two steps: finding the optimal number of clusters and performing spectral clustering. In this section, we explain the spectral clustering method in more detail.

Given a similarity matrix, the spectral clustering method first converts the matrix into a similarity graph [47] $G=(V, E)$, with a set of vertices $V$ and set of edges $E$. In this graph, each vertex corresponds to a data point and, when two vertices are connected, the edge is weighed by their similarity. The similarity value, i.e., the weight of an edge, should be high when the two vertices connected by the edge are in the same cluster, and low when they are not. Three ways to create a similarity graph are as follows:

- $\epsilon$-neighborhood graph: Two points are connected together with an edge if their distance is less than $\epsilon$.

- $k$-nearest neighbor graph: Two points are connected together if they are in the "neighborhood" of each other, where the neighborhood of a point is composed of the closest $k$ other points. 
- Fully connected graph: Two points are connected together if they have a positive similarity value.

After defining the similarity graph with one of the three schemes described above, we compute the Laplacian matrix of the graph. The Laplacian matrix of a graph is calculated using the following equation [13]:

$$
L=D-A,
$$

where $D$ is the degree matrix and $A$ is the adjacency matrix of the graph. An adjacency matrix defines the connectivity of a finite graph and is given by:

$$
A:=\left\{\begin{array}{l}
A_{i j}=1, \quad \text { if there is an edge between vertices } i \text { and } j, \\
A_{i j}=0, \quad \text { if there is no edge between vertices } i \text { and } j, \\
A_{i i}=0 .
\end{array}\right.
$$

An adjacency matrix basically captures whether or not pairs of vertices are connected to each other. If a pair of vertices is connected, the value of the corresponding matrix entry is one. Otherwise, it is zero.

The degree matrix provides information about the number of edges that a vertex is connected to, i.e., the degree of the vertex, and is given by:

$$
D:= \begin{cases}D_{i j}=\operatorname{deg}\left(v_{i}\right), & \text { if } \mathrm{i}=\mathrm{j} \\ D_{i j}=0, & \text { otherwise }\end{cases}
$$

where $\operatorname{deg}\left(v_{i}\right)=\sum_{j} A_{i j}$ indicates the number of edges connected to $v$.

The spectral clustering method can also use an affinity/similarity matrix in place of a weighted adjacency matrix. In this case, we assume that the input is a dense graph where all points connect to all other points, and the weights indicate the similarity between pairs of points. For our implementation, we make use of the similarity matrix produced using the Gaussian kernel described in Section 3.4.

As shown by Equation 3.6, the Laplacian matrix can be calculated by subtracting the adjacency matrix from the degree matrix. We then compute the eigenvalues $\lambda$ and eigenvectors $x$ of the Laplacian matrix. The eigenvalues and eigenvectors of a matrix $M$ satisfy the following equation: 


$$
M x=\lambda x
$$

When Equation 3.9 is satisfied for an eigenvalue $\lambda$, then the corresponding eigenvector is $x$. In the case of spectral clustering, $M$ is the Laplacian matrix $L$.

We find the desired number of clusters $k$ using the eigengap heuristic which is explained in the section 3.5.1. Given $k$, we calculate the first $k$ eigenvectors, specifically, the first $k$ smallest non-null eigenvalues. By stacking these eigenvectors, we create a new matrix $U_{n \times k}$ where the number of rows $n$ is the number of points to be clustered and the number of columns $k$ is the number of selected eigenvectors. To obtain the clusters, we represent each point $i$ by the $i_{\text {th }}$ row of $U$, and then cluster the points using the k-means clustering method, where the similarities among points is given by the $L_{2}$ norm of the rows. This technique is effective because the original data points have now been projected onto an embedding space where the clustering problem can be solved more easily (the spectral domain).

After clustering, all the points assigned to the same cluster compose one instance. Thus, the final output of the method is a set of instances for each category label.

\subsubsection{Finding the number of Clusters}

To find optimal number of clusters, we use a method called the eigengap heuristic. Eigengap denotes the "gap" or distance between consecutive eigenvalues.

Given a similarity matrix, we compute the eigenvalues and eigenvectors of the Laplacian matrix derived from the similarity matrix, as described in the previous section. Then, the index of the eigenvalue that has the largest distance to its consecutive eigenvalue is chosen as the optimal number of clusters. That is, if the distance between eigenvalues $\lambda_{1}, \ldots, \lambda_{k}$ is small compared to the distance between $\lambda_{k}$ and $\lambda_{k+1}$, then $k$ is selected as the number of clusters. 


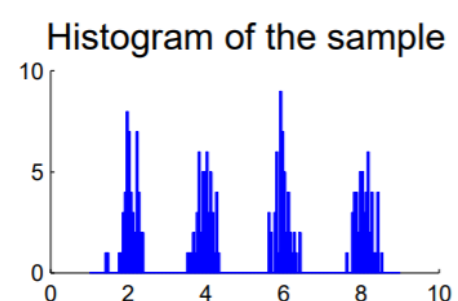

Eigenvalues

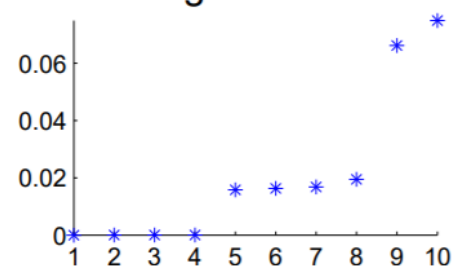

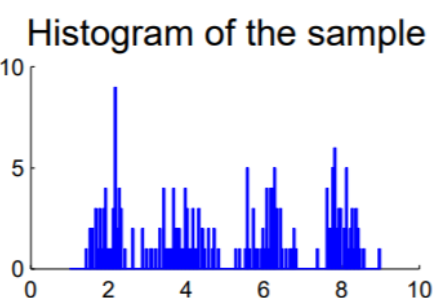

Eigenvalues

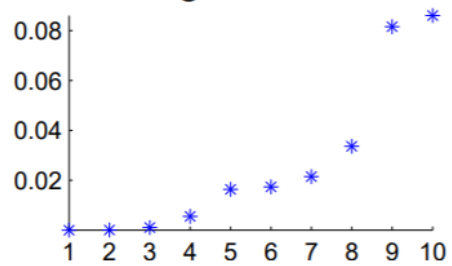

Histogram of the sample

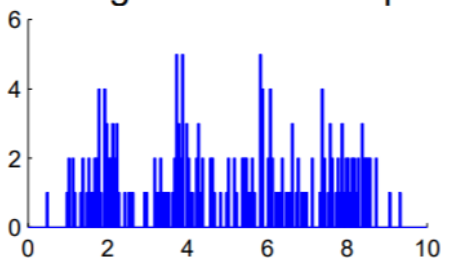

Eigenvalues

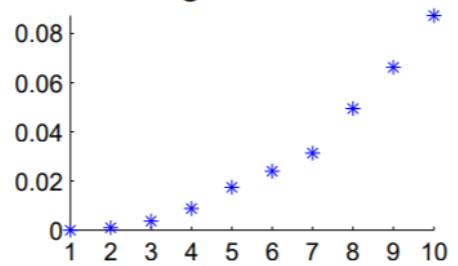

Figure 3.6: Eigengap heuristic illustration. Image source: Von Luxburg [47].

The intuition behind this method is illustrated in Figure 3.6, where three samples of data in the form of histograms are given in the first row. In the first column, we see that the eigenvalues clearly reflect the gap between the connected components of the histogram. The first $k=4$ eigenvalues are 0 and then the next value is greater than that, creating a "gap" at the expected number of clusters. There is also a small gap between the fourth and fifth eigenvalue, reflecting a possible grouping into 5 clusters. However, in the examples in columns 2 and 3, where the components of the data overlap, the gap between the eigenvalues is not as clear. An analysis of this heuristic [47] demonstrates that, given a well defined set of connected components, the eigengap heuristic provides the exact number of clusters, while the more ambiguous the dataset, the more unclear results will be produced.

\subsection{Constrained Clustering}

To further improve the instance segmentation computed automatically, we optionally allow the user to refine the results. To enable the user to perform this refinement without requiring significant manual effort, we use a constrained clustering method, where the user defines points that must-link and that cannot-link. Since the user will be providing input to the clustering, we first ask the user to specify the correct number of clusters that should be obtained by the method. Next, the user draws free-form curves around two sets of points $P$ and $Q$ according to a view of the segmentation 
result of the input point cloud. A set is defined as all the points inside of a given curve. Then, the user specifies whether the points in $P$ and $Q$ must-link or cannotlink. Specifically, we use Lasso selector [33] in the python library to allow the user to draw the curves.

To define the constraints, we modify the similarity matrix values related to the points in the two sets. We modify an entry of the similarity matrix to 1 if the points must link (i.e., we assign the maximum similarity to the points), and to 0 if they cannot link (minimum similarity):

$$
S(p, q)= \begin{cases}1, & \text { if must-link } p \text { and } q, \\ 0, & \text { if cannot-link } p \text { and } q,\end{cases}
$$

where $S$ is the similarity matrix derived from the distance matrix with the Gaussian kernel, and $p \in P$ and $q \in Q$. The assignment in Equation 3.10 is repeated for all pairs of points of $P$ and $Q$. We use the values 0 and 1, because after applying the Gaussian kernel to the distance matrix, the values become normalized, with 1 indicating the most similar entries and 0 indicating the dissimilarity.

In our experiments, we use mainly cannot-link constraints as most of our instances are clustered together as one instance that needs to be split into smaller instances, given that the similarity matrix is formed on the basis of the points being in the same category.

As illustrated in the example in Figure 3.7, given an input set of chairs, the user draws curves around two sets of points. We select all the points encircled by the drawings in the first set and the second set and make the similarity between them 0 . The output shows that the set of instances are now considered separate from each other. The final output after five rounds of user intervention is shown in Figure 3.8.

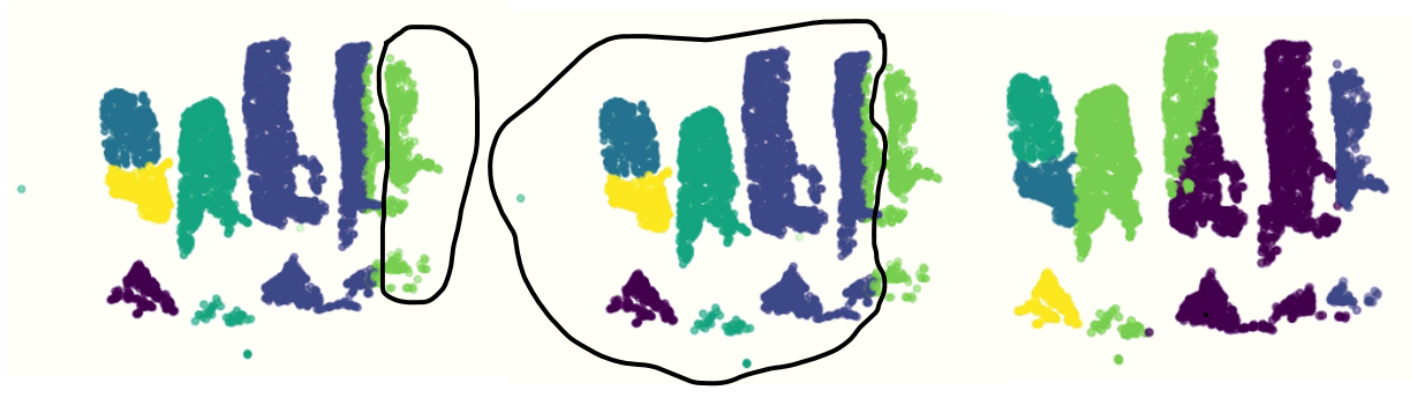

Figure 3.7: Use of the Lasso tool to circle points for cannot-link constraints. 


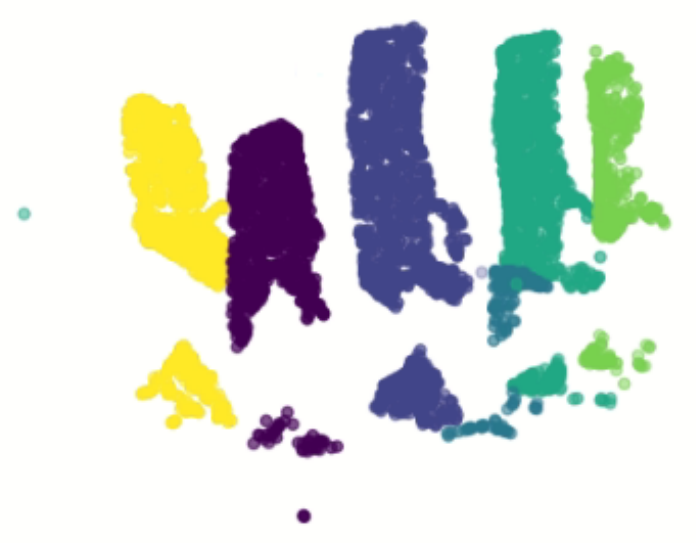

Figure 3.8: Final output of constrained clustering for chairs.

At first, it may seem that much user intervention is required with this method, taking much effort and time. However, note that adding constraints to the clustering often improves the result not only for the selected points, but also for other points connected to the selected points by their similarity. For example, as shown in Figure 3.9, we circle one instance (first column) and then identify the points that should not belong to this instance (second column). The similarity between those points and the instance in focus is set to 0 , and so the outer points are identified as outliers, while the instance is cleaned up (third column). In addition, the result for another table (in blue, on the top-right) is also cleaned up, although it was never explicitly circled.
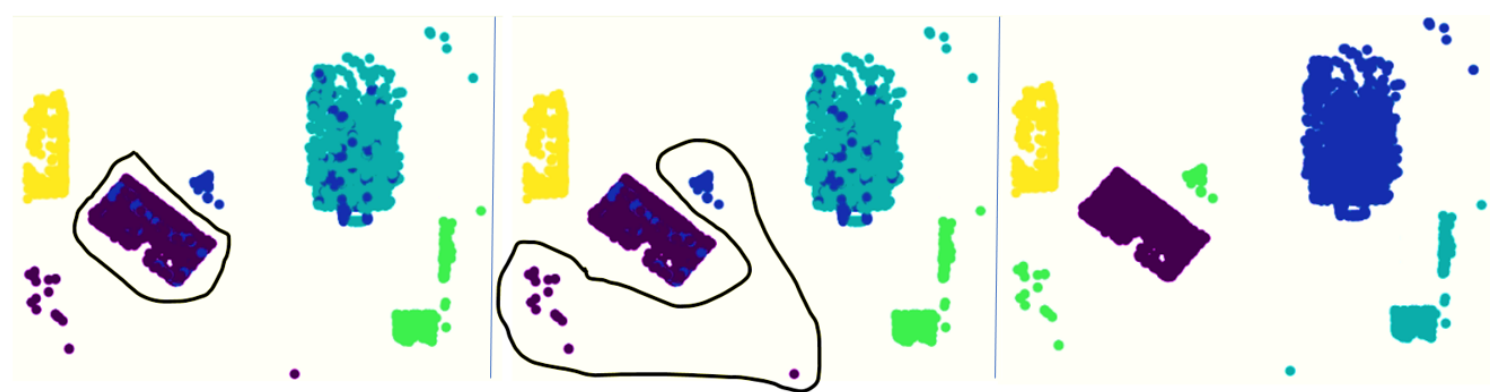

Figure 3.9: Benefit of constrained clustering. 


\subsection{Summary}

In this section, we summarize the entire instance segmentation method in the form of pseudo-code, shown in Algorithm 1.

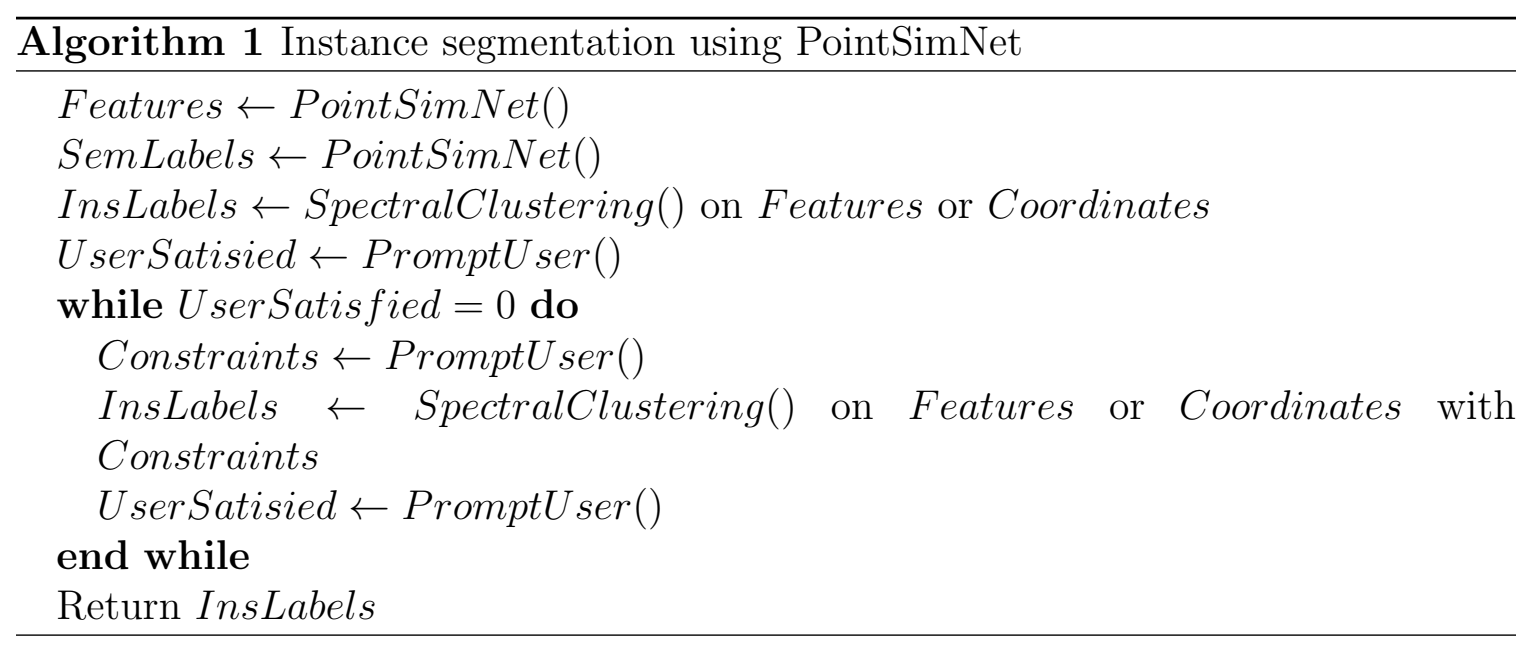




\section{Chapter 4}

\section{Results and Evaluation}

This chapter describes implementation details and presents the evaluation of our model. In Section 4.1, we describe the two datasets that we use for our evaluation. In Section 4.2, we list all the training parameters involved in the implementation of our method. In Section 4.3, we describe our evaluation criteria and how evaluation measures are calculated. Our main results are divided into two sections: quantitative results in Section 4.4, which evaluates the method in terms of measures such as Average Precision, and qualitative results in Section 4.5, which analyzes visual results. Section 4.6 describes the quantitative and qualitative results for constrained clustering. Lastly section 4.7 specifies the time analysis for our model.

\subsection{Datasets}

In this chapter, we evaluate the performance of our method on two different datasets, which we denote with the acronyms "S3DIS" and "CIMS".

\subsubsection{Stanford Dataset (S3DIS)}

The Stanford Large-Scale 3D Indoor Spaces Dataset (S3DIS) [4] is a well-known dataset used for evaluating scene-level segmentation methods. This dataset has 3D indoor scans that are split into six different areas, where each area contains multiple rooms. The training set is composed of Areas 1-5, while Area 6 is a dedicated test area. All the areas together contain a total of 271 rooms. The objects in the rooms are classified into 13 different categories: ceiling, floor, wall, chair, table, window, door, sofa, bookcase, beam, column, board, and clutter. The points are labeled from 
0-12 depending on which category they belong to. Each point in each point cloud also contains $x, y, z, r, g$, and $b$ attributes, where $x, y$, and $z$ are the 3D coordinates of the point, and $r, g$, and $b$ are the RGB color values of the point, where each color component ranges between 0 and 255 .

\subsubsection{CIMS Dataset}

The Carleton Immersive Media Studio (CIMS) provided us with a heritage dataset of indoor rooms containing architectural assets such as light fixtures and fireplaces. The dataset has a total of 42 room scans, where 10 rooms do not have any furniture in them. The objects in the rooms have been classified into 12 different categories: ceiling, floor, baseboard, column, light fixture, fireplace, wall, chair, table, paitning, window, and door. The points are labeled from 0-11 depending on which category they belong to. In addition to the label, each point in each point cloud contains 9 attributes, including $x, y$, and $z$ attributes capturing the 3D coordinates of each point, $r, g$, and $b$ attributes which are the RGB color values between 0 and 255 of each point, and $n x, n y$, and $n z$ attributes which are the normals associated with each point.

\subsection{Training and Parameters}

This section lists the different parameters associated with the implementation and training of our model.

- Epochs: our training time is divided between learning a semantic classifier and learning the predictor of point features. We train for a total of 200 epochs for S3DIS. The first 100 epochs are used for training the semantic classifier, while the remaining 100 epochs are used for training both the semantic classifier and the feature predictor. For CIMS dataset, we train for 100 epochs with 50 epochs for semantic learning and 50 for similarity alongside semantic.

- Batch size: our batch size is fixed to 4 .

- Learning rate: learning rate is fixed to 0.001 .

- Optimizer: Adam optimizer is used to train our model. 
- Decay rate: we use a decay rate of 300000 .

- Decay step: we use a decay step of 0.5 .

- Input data attributes: For the S3DIS dataset, the attributes are coordinates and RGB colors. For the CIMS dataset, the attributes are coordinates, colors, and normals.

- Spectral clustering $\delta$ value for Gaussian kernel, used to convert the distance matrix to a similarity matrix: we set $\delta$ as the maximum of all the entries for the distance matrix derived from the learned point features, and set $\delta$ as $0.7 \times$ the maximum of all the pairwise point distances, when deriving the distance matrix from the point coordinates. We explain our process for selecting these values in Section 4.4.

\subsection{Evaluation measures}

We evaluate our results with the Average Precision (AP) measure. AP ranges from 0 to 1 and AP\% indicates multiplying the AP by 100 .

The calculation of Average Precision is based on the calculation of the IoU (Intersection over Union) between each ground-truth and predicted instance. The IoU can be calculated either with bounding boxes or sets of points. That is, given a set of points labeled as one instance, one option is to fit an axis-aligned bounding box to the points, and compute the intersection and union of this bounding box with the bounding box fit to a ground-truth instance. The other option is to calculate the intersection and union of the sets of predicted points and ground-truth points.

SGPN introduced the method for calculating IoU based on point sets. However, given that the bounding box method is considered a standard for evaluating object detection results, we adapted the calculation of IoU based on axis-aligned bounding boxes (AABBs) to the context of 3D point clouds. Although object oriented bounding boxes (OOBBs) can provide tighter boxes that bound the detected objects [16], similar to most of the literature, we use AABBs to bound the objects, as the computation of AABBs and their union and intersection is much faster than that of OBBs.

Typically, AABBs are calculated by simply finding the minimum and maximum coordinate value of all the points in the object, and then using them to define the extent of the bounding box. However, we compute the boxes with the following 
procedure, to avoid creating large boxes due to the presence of outlier points. To fit a bounding box to a set of points, we find the mean and standard deviation of the points along each axis, and set the bounding box size along each dimension as the mean plus and minus one standard deviation along the dimension. To evaluate the quality of the fit, we make sure that we do not have more than a certain percentage of the points outside of the calculated box. If this happens, then we recalculate the box by using the minimum and maximum coordinate value along each of the three canonical axes as the box boundaries.

To arrive at the percentage of points that are allowed to be outside of the box, which we call the percentage from now on, we performed an experiment where we varied the percentage and calculated the AP\% of the results on S3DIS. We experimented with different percentage values such as 10\%, 20\%,30\% and so on. We get an AP\% higher or equal to 28.1 with any value below 50 . The AP\% decreases to 21.18 when we change the percentage from 50 to 60 . Thus, we select $50 \%$ as the percentage in order to be conservative (allowing the maximum number of points outside the box while not harming the performance of the method).

Table 4.1 shows the number of boxes computed with the minimum and maximum as opposed to the mean and standard deviation, when the test based on the percentage is activated. These statistics show that, no more than $23 \%$ of the time, $50 \%$ of the points or more were found outside the box calculated by the mean and standard deviation, triggering the recalculation of the box with the minimum and maximum. 


\begin{tabular}{|c|c|c|c|}
\hline Min/Max & Std/Mean & Total & Percentage \\
\hline 4 & 20 & 24 & 16 \\
3 & 16 & 19 & 15 \\
3 & 17 & 20 & 15 \\
4 & 19 & 23 & 17 \\
4 & 13 & 17 & 23 \\
3 & 19 & 22 & 13 \\
3 & 17 & 20 & 15 \\
8 & 37 & 45 & 17 \\
6 & 24 & 30 & 2 \\
4 & 19 & 23 & 17 \\
\hline
\end{tabular}

Table 4.1: Statistics for 10 different S3DIS rooms showing the number of bounding boxes computed with the minimum and maximum versus the mean and standard deviation. "Percentage" denotes the percentage of boxes out of the total computed with the minimum and maximum.

Once we have the boxes for the ground-truth instance and the predicted instance, we compute their volumes of intersection and union. The IoU is given by dividing the volume of intersection by the volume of the union.

Regardless of the method of IoU calculation, an IoU of 1 indicates that the groundtruth and predicted instance overlap completely, while 0 indicates no overlap. If the IoU is greater than a threshold, which is 0.5 in most of the literature, then the instance is considered a True Positive (TP) (correct detection of a ground-truth instance). If the IoU is less than the threshold, then the instance is considered a False Positive (FP). An instance is considered a False Negative (FN) when there is no prediction for a ground-truth instance. We keep FNs as zero in our evaluation as we always have a prediction for a ground-truth instance.

After the calculation of TPs and FPs, we calculate precision and recall. Precision is the number of true positive instances amongst all the instances found. Recall is the total amount of positive instances that are labeled correctly [34]. The formulas 
are given by [12]:

$$
\begin{gathered}
\text { Precision }=\frac{T P}{T P+F P} . \\
\text { Recall }=\frac{T P}{T P+F N} .
\end{gathered}
$$

Precision and recall come with a trade-off between each other. That means that if the precision value is increased due to a change of parameters in the method, then the recall value will decrease, and vice-versa. By calculating the recall and precision of all ground-truth instances, we are able to form a precision-recall curve.

Average precision (AP) is then defined as the area under the precision-recall curve. This curve is not always monotonically decreasing which means that precision and recall can oscillate up and down. To get rid of the 'wiggles' [32], Interpolated Precision (IP) has been introduced to allow the calculation of a meaningful area under the curve [28]. Basically, the curve is traced for each recall level ranging from 0 to 1 , and the maximum precision to the right of a point on the curve is chosen as the precision for the point. The formula is given by:

$$
I P(r)=\max _{r^{\prime} \geq r}\left(p\left(r^{\prime}\right)\right),
$$

where $r$ is the recall level ranging from 0 to $1, p$ is the precision value for the given recall value $r^{\prime}$, and $r^{\prime}$ is the adjusted recall value.

A standard way of using the IP in the literature is the 11-point Interpolated Precision [32], where 11 recall values are used, which start from 0 and are incremented 0.1 at every step until reaching 1 . After that, we find the average precision by calculating the arithmetic mean of all the interpolated precision values, which is given by:

$$
A P=\frac{1}{11} \sum_{r \in 0,0.1, \ldots, 1} I P(r) .
$$

The entire evaluation procedure is summarized in Algorithm 2. 


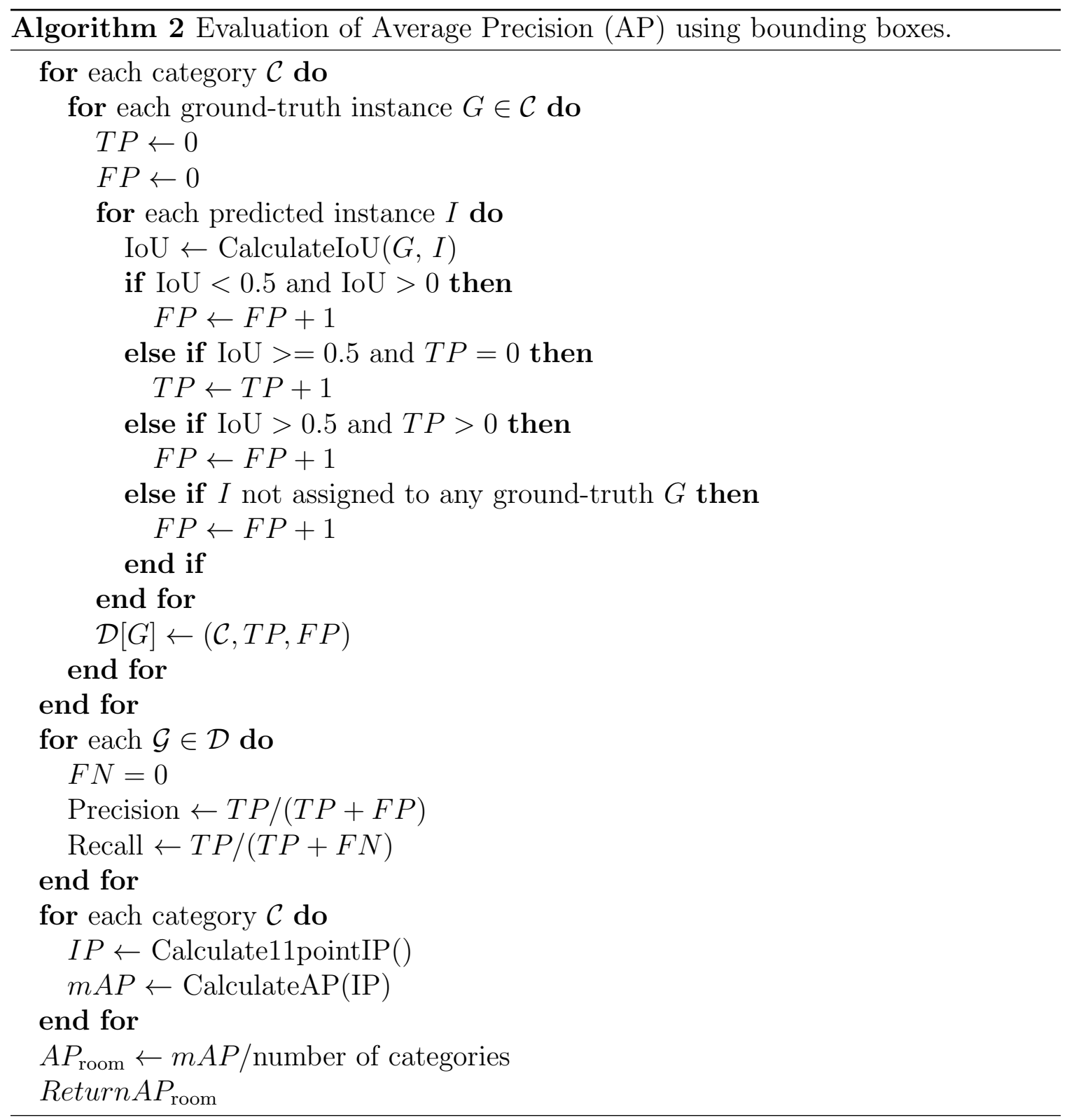




\subsection{Quantitative results}

This section describes the quantitative evaluation of semantic segmentation and instance segmentation. The quantitative evaluation of the constrained clustering is discussed in Section 4.6. As we do not have instance labels for the CIMS dataset, our quantitative evaluation for instance segmentation focuses only on the S3DIS dataset.

Table 4.2 and 4.3 show the evaluation of the semantic segmentation quality obtained with our method, compared to the original PointNet. As shown in Table 4.3, although the network learns to predict point features that capture the similarity of points, the network can still also learn a semantic classifier from these features which maintains the accuracy of PointNet.

\begin{tabular}{|c|c|c|}
\hline & Ours & PointNet \\
\hline Stanford Dataset & 81.8 & 79.66 \\
CIMS dataset & 79.7 & 76.12 \\
\hline
\end{tabular}

Table 4.2: Semantic segmentation results on S3DIS and CIMS datasets. These values represent the labeling accuracy, which is the number of correctly predicted points divided by the total number of points.

\begin{tabular}{|l|c|c|}
\hline & Ours & PointNet \\
\hline Stanford Dataset & 59.2 & 47.71 \\
\hline
\end{tabular}

Table 4.3: Semantic segmentation results on S3DIS. The measure is the average IoU over 13 classes.

For the CIMS dataset, we trained the model for a total of 100 epochs. We train the semantic classifier for the first 50 epochs and then train the network that predicts point features with a similarity structure for the last 50 epochs. For the S3DIS dataset, we train the model for 200 epochs. The semantic classifier is trained in the first 100 epochs and point feature similarity in the remaining 100 .

As mentioned in Chapter 3, after obtaining the point features, we perform instance segmentation using spectral clustering. The improvement in different categories achieved by our method is without any training with instance labels. 
Table 4.4 shows the instance segmentation results of our method applied on the S3DIS dataset. We compare our results when performing spectral clustering based on coordinates or predicted features. We also compare to the results of the SGPN method [50]. The results in the table are calculated using the intersection and union of point sets, similar to SGPN paper. We see that in categories such as ceiling, floor, sofa, and board our method outperforms SGPN with their measurement.

\begin{tabular}{|c|c|c|c|c|c|c|c|c|c|c|c|c|c|}
\hline & Mean & ceiling & floor & wall & beam & column & window & door & table & chair & sofa & bookcase & board \\
\hline SGPN & $\mathbf{4 7 . 8 4}$ & 73.35 & 97.65 & $\mathbf{3 5 . 6 6}$ & $\mathbf{9 7 . 1 3}$ & $\mathbf{7 1 . 1 1}$ & $\mathbf{5 1 . 9 4}$ & $\mathbf{5 6 . 8 8}$ & $\mathbf{1 8 . 2 8}$ & $\mathbf{2 8 . 1 2}$ & 0 & $\mathbf{2 0 . 2 4}$ & 23.7 \\
Ours (Coordinates) & 29.56 & $\mathbf{8 0}$ & $\mathbf{1 0 0}$ & 2.81 & 39.79 & 10 & 23.78 & 30.58 & 13.08 & 9.86 & 5 & 13.87 & 25.91 \\
Ours (Features) & 31.82 & $\mathbf{8 0}$ & $\mathbf{1 0 0}$ & 2.38 & 45.45 & 1.88 & 33.53 & 43.21 & 9.31 & 2.14 & $\mathbf{5 . 1 7}$ & 9 & $\mathbf{4 9 . 8 1}$ \\
\hline
\end{tabular}

Table 4.4: Instance segmentation results on the S3DIS dataset. The metric is AP\% with IoU of 0.5 , and is calculated on sets of points as introduced by SGPN.

The calculation of AP\% introduced by SGPN, which is based on the IoU of point sets, is not a standard evaluation measure. Most object detection methods, which have their roots in computer vision methods handling images, use a calculation based on bounding boxes, as explained in Section 4.3. Thus, in Table 4.5, we also present the results of our method and SGPN using the bounding box calculation. We see that the bounding box method is a stricter measure than the calculation based on point sets, as the average accuracy is lower when compared to Table 4.4. Using this calculation of $\mathrm{AP} \%$, our methods based on spectral clustering of coordinates and features increase SGPN's AP by almost $20 \%$ points. The categories wall, column, chair, and board are the only ones where SGPN performs better than our method.

\begin{tabular}{|c|c|c|c|c|c|c|c|c|c|c|c|c|c|}
\hline & Mean & ceiling & floor & wall & beam & column & window & door & table & chair & sofa & bookcase & board \\
\hline SGPN & 8.13 & 11.2 & 8.13 & $\mathbf{8 . 6 9}$ & 9.37 & $\mathbf{2 . 3 9}$ & 19.35 & 7.48 & 8.23 & $\mathbf{1 3 . 2}$ & 0 & 4.6 & $\mathbf{4 . 8 4}$ \\
Ours (Coordinates) & 27.6 & $\mathbf{8 6 . 5}$ & $\mathbf{9 0 . 9}$ & 0 & $\mathbf{2 8 . 8 2}$ & $\mathbf{2 . 3 9}$ & $\mathbf{4 3 . 8}$ & 25.9 & $\mathbf{3 6 . 6}$ & 2.27 & $\mathbf{2 . 0 6}$ & $\mathbf{1 1 . 0 8}$ & 1.16 \\
Ours (Features) & $\mathbf{2 8 . 1}$ & $\mathbf{8 6 . 5}$ & $\mathbf{9 0 . 9}$ & 0 & 27.1 & 0 & 37.337 & $\mathbf{5 2 . 3}$ & 32.57 & 1.13 & 0 & 8.49 & 1.16 \\
\hline
\end{tabular}

Table 4.5: Instance segmentation results on the S3DIS dataset. The metric is AP\% with IoU of 0.5 , and is calculated using the bounding box method.

The results in Tables 4.4 and 4.5 are calculated with an IoU threshold of 0.5. We also evaluate the results with IoU thresholds of $0.25,0.5$, and 0.75 on the S3DIS dataset in comparison with SGPN. We show these results in Tables 4.6 and 4.7. Using 
the point measure, SGPN shows better results than our method, while our method performs better according to the evaluation using the bounding box method.

\begin{tabular}{|c|c|c|c|}
\hline & $A P_{0.25}$ & $A P_{0.5}$ & $A P_{0.75}$ \\
\hline SGPN & $\mathbf{6 1 . 0 3}$ & $\mathbf{4 7 . 8 4}$ & $\mathbf{3 0 . 4}$ \\
Ours (Features) & 46.2 & 29.56 & 17.9 \\
Ours (Coordinates) & 44.38 & 31.82 & 19.45 \\
\hline
\end{tabular}

Table 4.6: Instance segmentation results on the S3DIS dataset. The metric is AP\% with different IoU thresholds, and is calculated on sets of points over 12 categories.

\begin{tabular}{|c|c|c|c|}
\hline & $A P_{0.25}$ & $A P_{0.5}$ & $A P_{0.75}$ \\
\hline SGPN & 11.1 & 8.13 & 4.6 \\
Ours (Features) & $\mathbf{3 7 . 7 4}$ & $\mathbf{2 8 . 1}$ & $\mathbf{2 0 . 3 2}$ \\
Ours (Coordinates) & 37.15 & 27.6 & 19.6 \\
\hline
\end{tabular}

Table 4.7: Instance segmentation results on the S3DIS dataset. The metric is AP\% with different IoU thresholds, and is calculated using the bounding box method over 12 categories.

To analyze in more detail the difference between the results of PointSimNet and SGPN, we selected a couple of rooms and show the AP\% computed individually for each room. The results are shown in Tables 4.8 and 4.9 with the different manners of calculating the AP\%. For our method, clustering on learned features provides a better AP\% than on coordinates, as features provide more knowledge about the points and their categories than just their Euclidean distance.

For Office 6, SGPN outperforms our method by $3 \%$ points and for Office 32, by $11 \%$ points. For Office 22 , our method outperforms SGPN by $20 \%$ points. For Office 31 and Office 29, our method outperforms SGPN by $7 \%$ points and $5 \%$ points respectively. These results are using the calculation of IoU by point method. 


\begin{tabular}{|c|c|c|c|}
\hline & Ours (Features) & Ours (Coordinates) & SGPN \\
\hline Office 6 & 35.38 & 38.85 & $\mathbf{4 1 . 0 6}$ \\
Office 22 & $\mathbf{3 4 . 2 3}$ & 33.08 & 14.63 \\
Office 31 & $\mathbf{4 5 . 3 8}$ & 40.77 & 38.72 \\
Office 32 & 46.15 & 30.77 & $\mathbf{5 9 . 4 2}$ \\
Office 29 & $\mathbf{5 3 . 0 8}$ & 46.92 & 48.41 \\
\hline
\end{tabular}

Table 4.8: Instance segmentation results on the S3DIS dataset for different rooms. The metric is AP\% with IoU threshold of 0.5, and is calculated using sets of points.

Using our method of calculation based on bounding boxes, we obtain better results for all the selected rooms, with almost twice the AP\% that SGPN obtains on all the rooms. The visual comparison of these results is shown in Section 4.5.

\begin{tabular}{|c|c|c|c|}
\hline & Ours (Features) & Ours (Coordinates) & SGPN \\
\hline Office 6 & $\mathbf{3 7 . 8 7}$ & 30.3 & 5.58 \\
Office 22 & $\mathbf{1 9 . 2}$ & 16.52 & 5.25 \\
Office 31 & $\mathbf{4 7 . 7 2}$ & 36.36 & 9.48 \\
Office 32 & $\mathbf{2 2 . 7 2}$ & $\mathbf{2 2 . 7 2}$ & 7.81 \\
Office 29 & $\mathbf{2 2 . 7 2}$ & 15.15 & 13.5 \\
\hline
\end{tabular}

Table 4.9: Instance segmentation results on the S3DIS dataset for different rooms. The metric is $\mathrm{AP} \%$ with IoU threshold of 0.5 , and is calculated using the bounding box method.

Some recent papers that perform instance segmentation with supervised machine learning evaluate their models with mean precision (denoted mPrec) and mean recall ( $m$ Recall) which are basically calculation of average of the precision and recall values described above. These methods also perform the evaluation on Area 5 from the S3DIS dataset rather than Area 6 . Area 5 is a more difficult case than Area 6, as it does not reside in the same building as all the other areas [52]. Thus, the objects 
differ from other areas. Table 4.10 shows the results for mPrec and mRecall for Area 5 with our method, SGPN, and other recent methods.

\begin{tabular}{|c|c|c|}
\hline Method & mPrec & mRecall \\
\hline Ours (coordinates) & 27.06 & 21.69 \\
Ours (features) & 27.5 & 21.59 \\
SGPN [50] & 36 & 28.7 \\
ASIS [48] & 55.3 & 42.4 \\
PointGroup [24] & 61.9 & $\mathbf{6 2 . 1}$ \\
\hline OccuSeg [21]* & $\mathbf{7 2 . 8}$ & 60.3 \\
\hline
\end{tabular}

Table 4.10: Instance segmentation results on the S3DIS dataset on Area 5. The method marked with $*$ is evaluated with 6 -fold cross validation. The metrics are mPrec and mRecall with IoU threshold of 0.5 , calculated using the point set method.

While our method provides lower precision and recall values, all of the other methods use instance label information for training, while we do not. The recent methods OccuSeg and PointGroup also first voxelize the point clouds and then perform instance segmentation, which requires more memory and processing time. In this regard, our approach to instance segmentation allows users to improve the result with constraints, as shown in Tables 4.16 and 4.17

As discussed in Section 3, we selected the $\delta$ value for the Gaussian kernel in an experimental manner, trying different thresholds of the mean and maximum values of the distance matrix on the training data. Specifically, we considered the constants $0.3,0.5,0.7,0.9$, and 1 multiplied by the mean and maximum of all entries of the distance matrix. The results of all thresholds are shown in Table 4.11. 


\begin{tabular}{|c|c|c|c|c|c|c|c|c|c|c|c|c|c|}
\hline & Mean & ceiling & floor & wall & beam & column & window & door & table & chair & sofa & bookcase & board \\
\hline Coordinates with $\max$ & 26.25 & 86.5 & 90.9 & 0 & 15.52 & 0 & 32.46 & 46.53 & 30.6 & 5.6 & 0 & 5.54 & 1.16 \\
\hline Coordinates with $\max * 0.3$ & 26.2 & 86.5 & 90.9 & 0 & 23.2 & 2.39 & 19.4 & 28.1 & 32.19 & 17.04 & 2.06 & 11.08 & 1.94 \\
\hline Coordinates with $\max * 0.5$ & 25.08 & 86.5 & 90.9 & 0 & 25.49 & 0 & 21.1 & 23.8 & 40.5 & 3.4 & 1.37 & 6.65 & 1.16 \\
\hline Coordinates with $\max * 0.7$ & 27.6 & 86.5 & 90.9 & 0 & 28.82 & 2.39 & 43.8 & 25.9 & 36.6 & 2.27 & 2.06 & 11.08 & 1.16 \\
\hline Coordinates with $\max * 0.9$ & 27.4 & 86.5 & 90.9 & 0 & 19.9 & 0 & 37.33 & 47.6 & 35.2 & 4.54 & 0 & 6.65 & 1.16 \\
\hline Coordinates with mean & 26.7 & 86.5 & 90.9 & 0 & 22.5 & 2.39 & 17.6 & 31.3 & 29.1 & 20.45 & 0 & 7.95 & 12.3 \\
\hline Coordinates with mean $* 0.3$ & 23.4 & 86.5 & 90.9 & 1.08 & 14.7 & 4.78 & 3.03 & 15.8 & 29.5 & 17.04 & 0 & 13.2 & 4.58 \\
\hline Coordinates with mean $* 0.5$ & 25.6 & 86.5 & 90.9 & 0 & 19.9 & 5.26 & 17.67 & 27.7 & 33.14 & 15.34 & 0 & 7.57 & 3.58 \\
\hline Coordinates with mean $* 0.7$ & 25.6 & 86.4 & 90.9 & 0 & 32.5 & 3.68 & 14.1 & 24.02 & 32.3 & 7.95 & 0 & 8.71 & 6.6 \\
\hline Coordinates with mean $* 0.9$ & 25.8 & 86.5 & 90.9 & 0 & 23.2 & 3.58 & 11.6 & 28.86 & 31.8 & 10.2 & 0 & 14.7 & 7.97 \\
\hline Features with max & 28.1 & 86.5 & 90.9 & 0 & 27.1 & 0 & 37.337 & 52.3 & 32.57 & 1.13 & 0 & 8.49 & 1.16 \\
\hline Features with $\max * 0.3$ & 26.49 & 86.5 & 90.9 & 0 & 21.5 & 0 & 37.33 & 49.24 & 21.09 & 1.136 & 0 & 8.13 & 1.94 \\
\hline Features with $\max * 0.5$ & 27.47 & 86.5 & 90.9 & 0.72 & 23.2 & 0 & 38.4 & 50.5 & 32 & 0 & 0 & 4.98 & 2.33 \\
\hline Features with $\max * 0.7$ & 27.47 & 86.5 & 90.9 & 0 & 24.02 & 0 & 38.96 & 50.14 & 32.57 & 0 & 0 & 5.17 & 1.359 \\
\hline Features with $\max * 0.9$ & 27.9 & 86.5 & 90.9 & 0.54 & 27.34 & 0 & 38.9 & 54.6 & 28.5 & 1.13 & 0 & 5.7 & 0.77 \\
\hline Features with mean & 24.5 & 86.5 & 90.9 & 0 & 15.8 & 0 & 34.09 & 38.9 & 18.25 & 1.13 & 0 & 7.76 & 1.16 \\
\hline Features with mean $* 0.3$ & 24.2 & 86.5 & 90.9 & 0 & 17.04 & 2.45 & 33.10 & 36.5 & 17.71 & 0 & 0 & 4.16 & 2.99 \\
\hline Features with mean $* 0.5$ & 24.27 & 86.5 & 90.9 & 0 & 16.99 & 1.196 & 31.11 & 37.69 & 16.51 & 0 & 0 & 5.98 & 4.27 \\
\hline Features with mean $* 0.7$ & 24.26 & 86.5 & 90.9 & 0 & 21.6 & 2.39 & 28.6 & 28.4 & 20.4 & 2.27 & 0 & 5.17 & 4.66 \\
\hline Features with mean $* 0.9$ & 24.14 & 86.5 & 90.9 & 0 & 20.87 & 1.196 & 29.22 & 34.70 & 21.96 & 0 & 0 & 2.29 & 1.94 \\
\hline
\end{tabular}

Table 4.11: Instance segmentation results on the S3DIS dataset showing experiments with different $\delta$ values in gaussian kernel. The metric is AP\% with IoU of 0.5 , and is calculated using the bounding box method.

We see in Table 4.11 that different values of $\delta$ provide the best results for different categories, with no value being optimal for all categories. For example, for the category wall, most of the results are close to an AP\% of 0 . However, when clustering on point features with the $\delta$ value of $\max ^{*} 0.5$, we obtain an $\mathrm{AP} \%$ of 1.08 . Another interesting case is with the door category, where we have $\mathrm{AP} \%$ as low as 15 when mean values are used to set $\delta$, while we can obtain an AP\% of 54.6 when using the maximum of the features. After this analysis, we selected max $\times 0.7$ as the most suitable for clustering of the S3DIS dataset based on point coordinates, and $\max \times 1$ for clustering based on point features, as these values give the best performance for the majority of classes. 


\subsection{Qualitative results}

In this section, we show and analyze visual examples of the results of our method for both datasets. We briefly discuss our results on semantic segmentation in comparison to SGPN and the ground-truth. Then, we compare the instance segmentation results of PointSimNet with features, PointSimNet with coordinates, and SGPN. As in Section 4.4, we have selected a few representative rooms from the S3DIS dataset to compare the results of instance segmentation: Office 6, Office 22, Office 31, Office 32, and Office 29. From the CIMS dataset, we selected rooms 484S and 601S.

\subsubsection{Semantic Segmentation results}

In this section, we analyze the semantic segmentation results for the S3DIS and CIMS datasets. The point colors in the pictures represent different object categories. We provide the ground-truth beside each result for reference. Note that we visualize only the points, drawn as spheres with a small radius.

For the S3DIS dataset, visual results of semantic segmentation are shown in Figure 4.1. For Office 2, the results of SGPN look slightly better than our results, when comparing to the ground-truth. On the other hand, for Office 8, our results look significantly better than those of SGPN, e.g., the prediction of walls, ceiling, and tables is more accurate. For SGPN, there are black patches in the regions corresponding to these classes, indicating that these regions were predicted as clutter, giving these rooms a messier look when compared to our results, which look cleaner. 

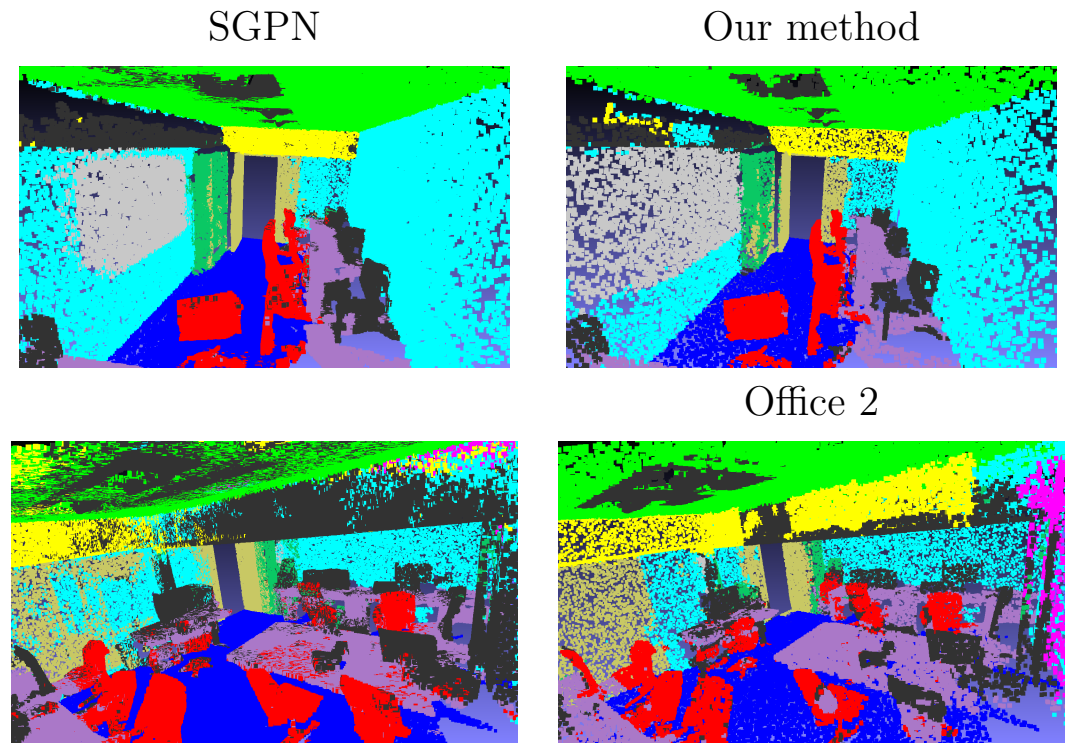

Office 2

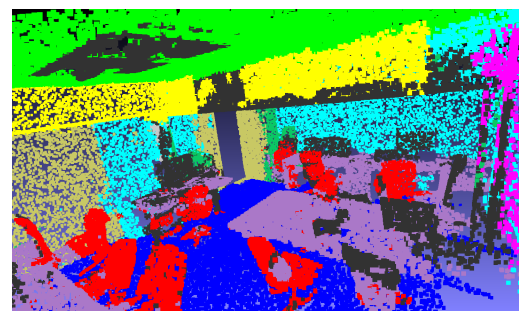

Office 8
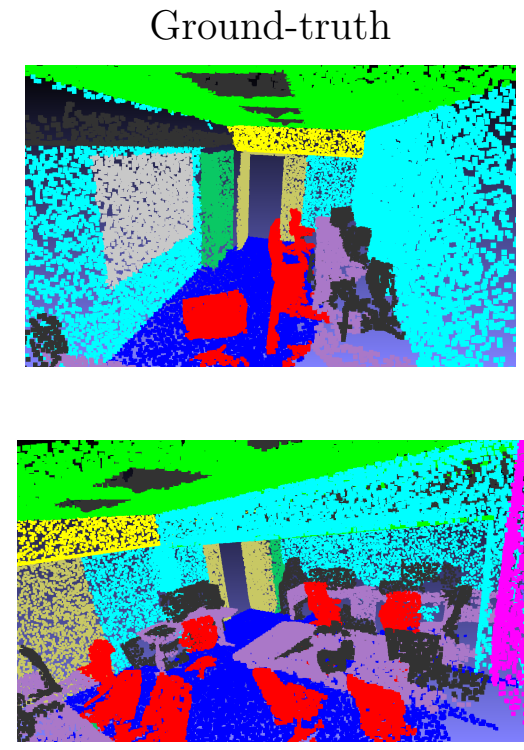

Figure 4.1: Semantic segmentation results on the S3DIS dataset.

For the CIMS dataset, visual results of semantic segmentation are shown in Figure 4.2. We show the results for Rooms 601, 484, and 371. Room 601 has no furniture. We see that the categories ceiling and floor are better predicted than the other categories. This is because these categories appear in every training room and have the same structure. On the other hand, categories such as windows and curtains are categorized as walls because not all training rooms contain windows. In addition, the structure of the windows in Room 601 differ from that in Rooms 484 and 371. In order to learn such a variety of structures, more examples need to be present in the training dataset. 

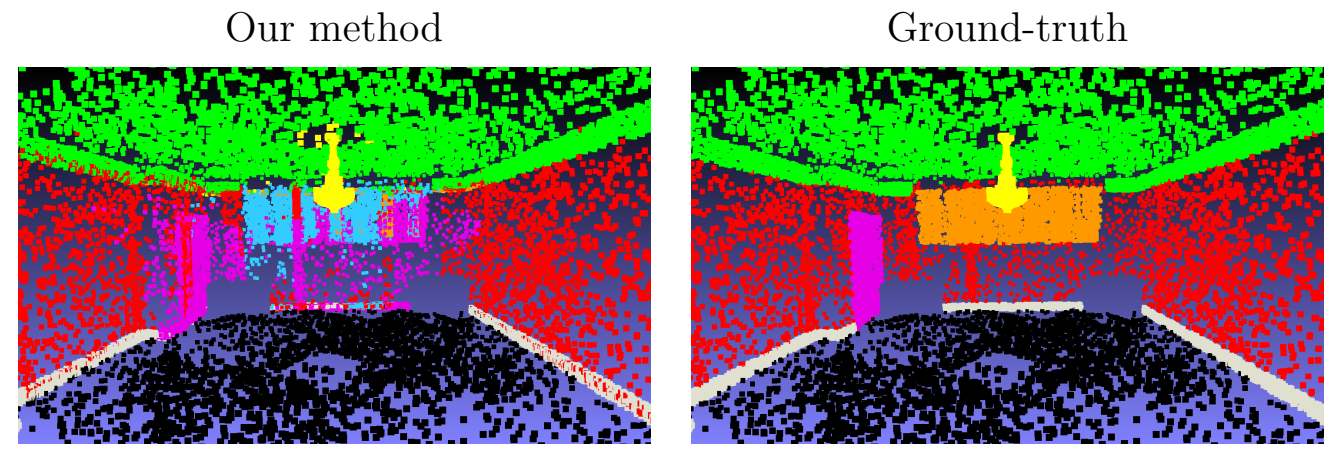

Room 601
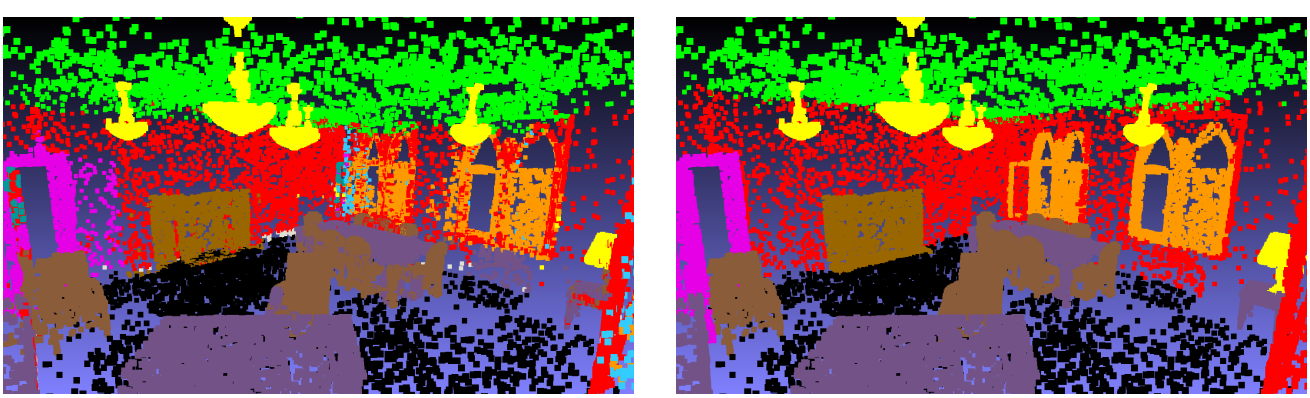

Room 484
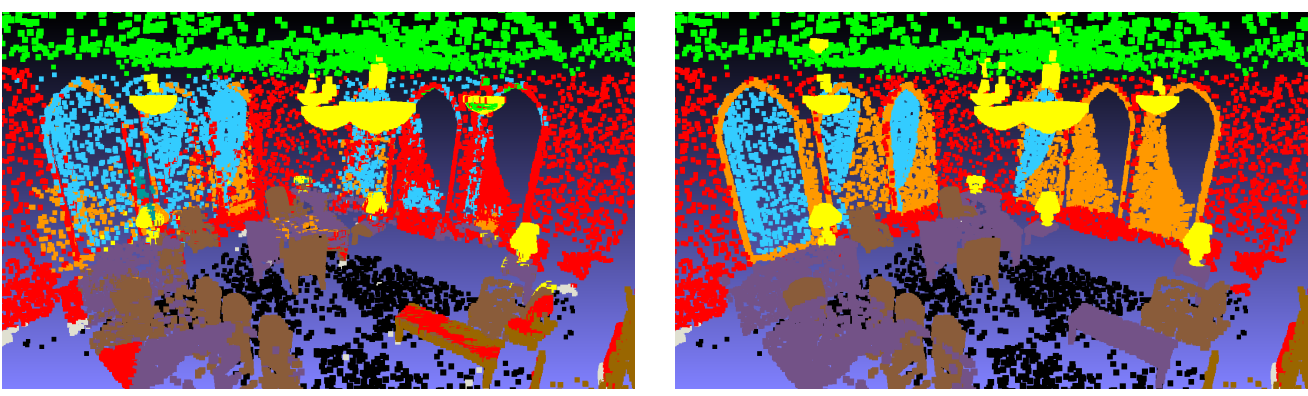

Room 371

Figure 4.2: Semantic segmentation results on the CIMS dataset.

Since the CIMS dataset has only 42 rooms in total, which is a small number of rooms compared to S3DIS, we train our model on S3DIS and then test on CIMS rooms, to demonstrate that the training set can benefit the segmentation of unseen datasets with similar structure. We remove the categories light fixture and fireplace from the CIMS dataset as these do not exist in S3DIS.

The results on the CIMS dataset with the model trained on S3DIS are shown in Figure 4.3. We see that the categories that have similar structure in both datasets, such as ceiling, floor, table, and walls, are segmented well. On the other hand, the 
CIMS dataset comprises heritage rooms and thus the architecture of windows and doors is completely different from that of modern offices, such as those present in S3DIS. Therefore, the categories door and windows are not segmented well.
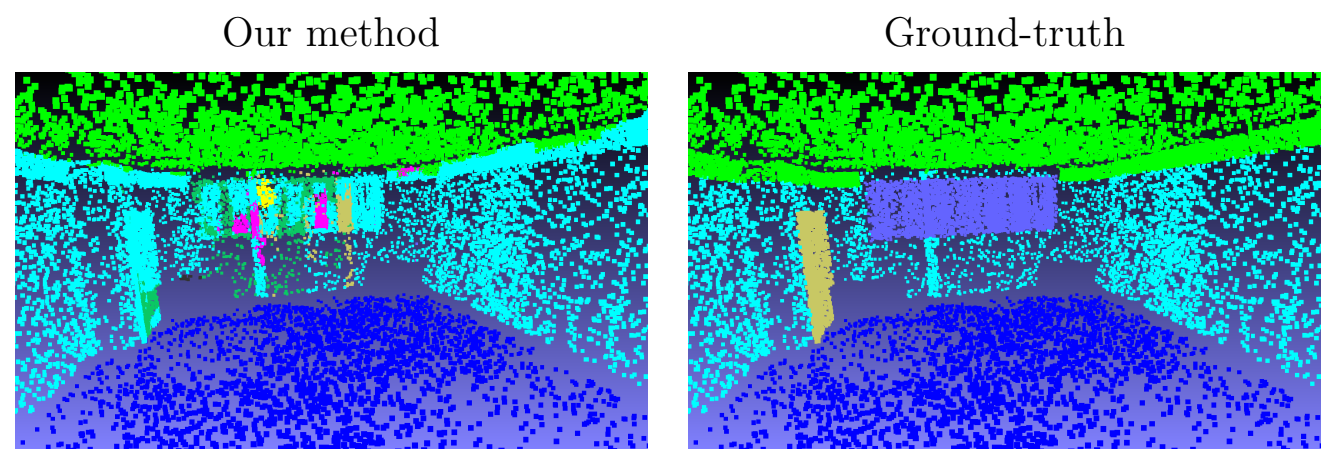

Room 601
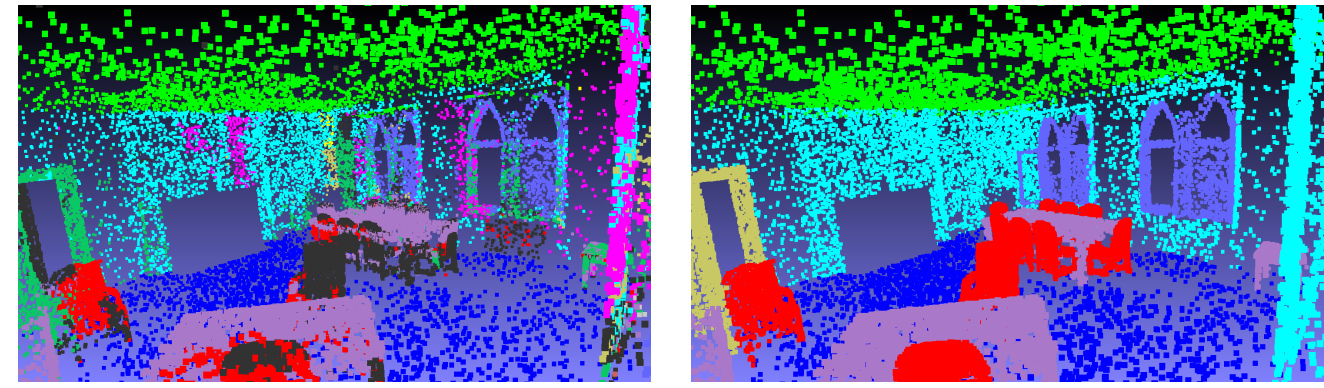

Room 484

Figure 4.3: Semantic segmentation results on the CIMS dataset with the model trained on the S3DIS dataset.

\subsubsection{Instance Segmentation results}

As mentioned in Section 4.4, for the S3DIS dataset, we selected five different rooms to compare the results of our instance segmentation method on point features and coordinates to SGPN. Here, we show the visual results for these rooms. Different colors indicate the different instances.

We notice that, in all the rooms, our results for instance segmentation with clustering on point features look better than those of SGPN. Especially for walls, we see that the results of SGPN have blocks of a different category combined with the walls. In our results, the accuracy for the category chairs is lower than SGPN. However, as we will discuss in Section 4.6, incorporating user input can improve our results for 
chairs in these rooms.

Figures 4.4 and 4.5 show the results of instance segmentation for Office 6 and Office 32. According to the statistics, the results of SGPN are more accurate for these rooms. However, visually our results look cleaner than those of SGPN, especially for Office 6, as the results of SGPN display patches of categories mixed together on the walls, tables and chairs.

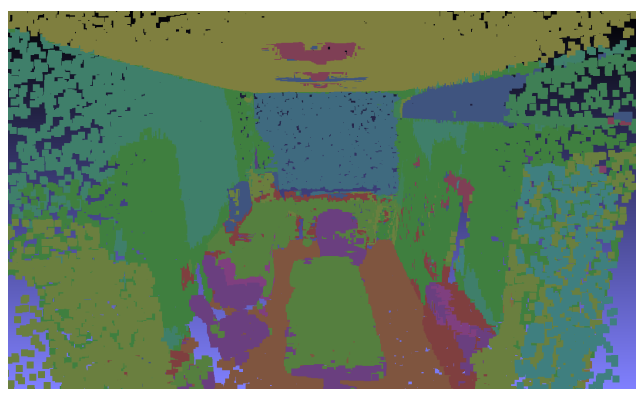

Clustering on features

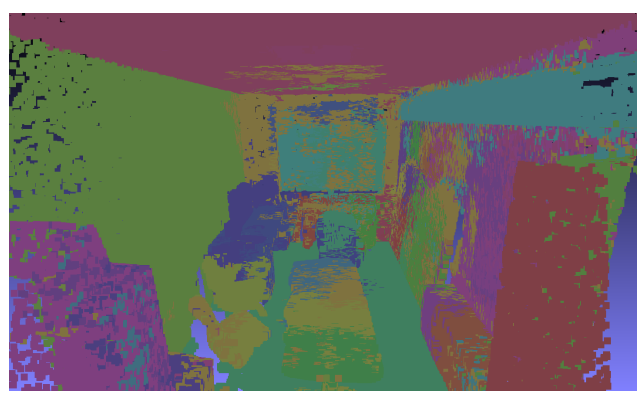

SGPN

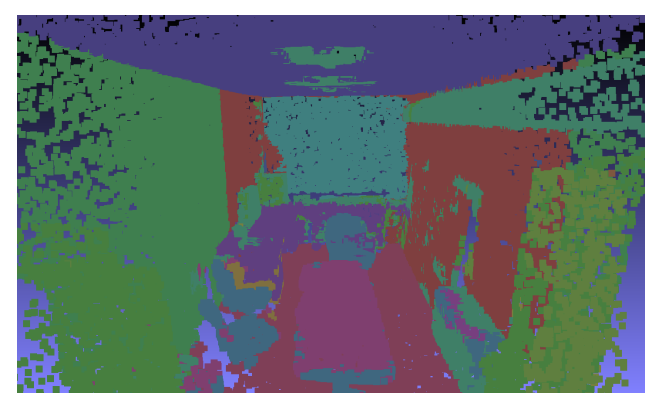

Clustering on coordinates

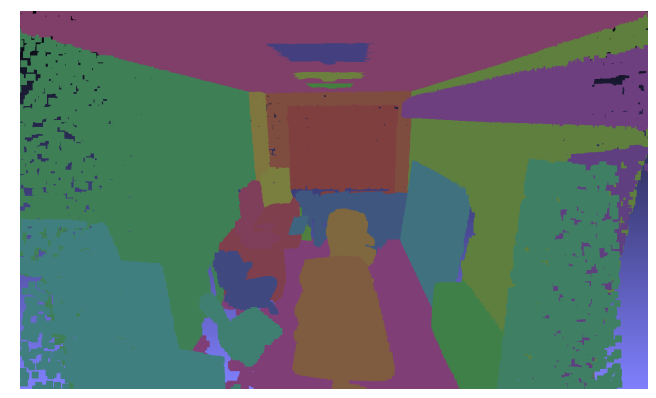

Ground-truth

Figure 4.4: Instance segmentation results for Office 6. 


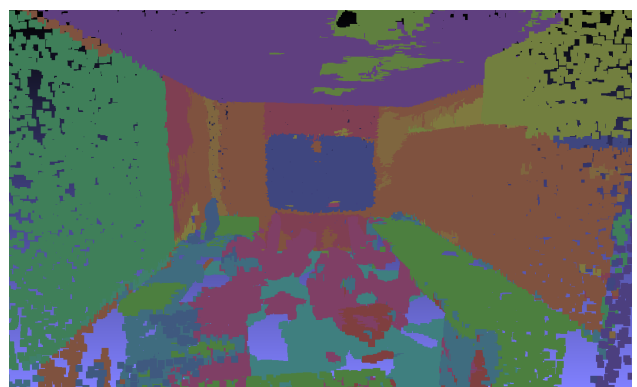

Clustering on features

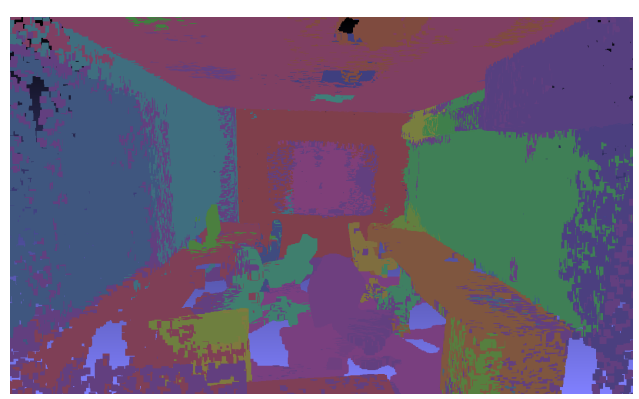

SGPN

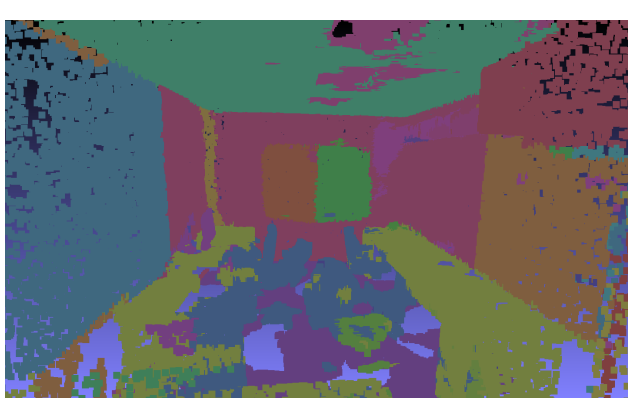

Clustering on coordinates

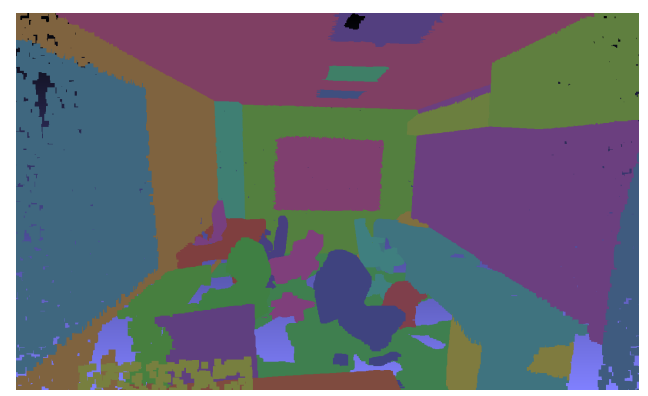

Ground-truth

Figure 4.5: Instance segmentation results for Office 32.

For Office 22, Office 31, and Office 29, our method quantitatively outperforms SGPN. We show the corresponding visual results in Figures 4.6, 4.7 and 4.8. Our improvement is visible in categories such as walls, ceiling, and furniture. 


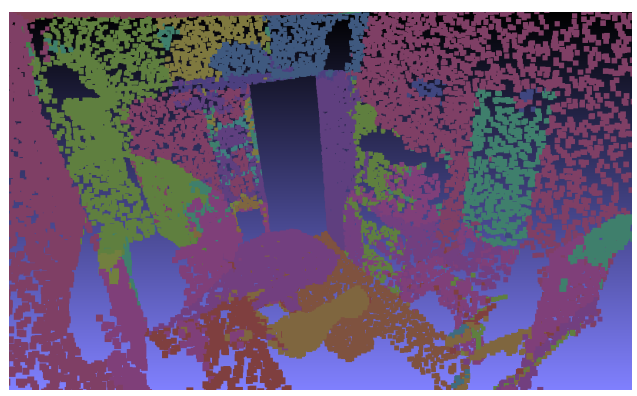

Clustering on features

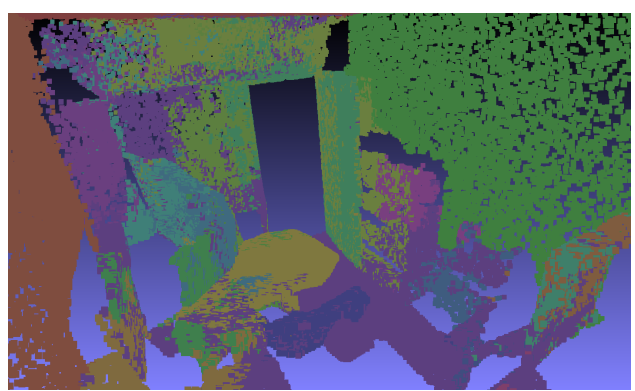

SGPN

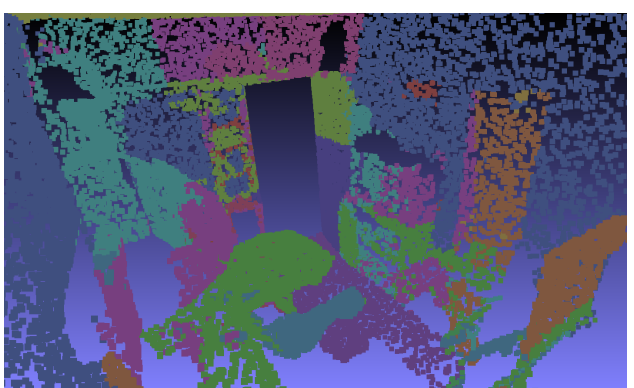

Clustering on coordinates

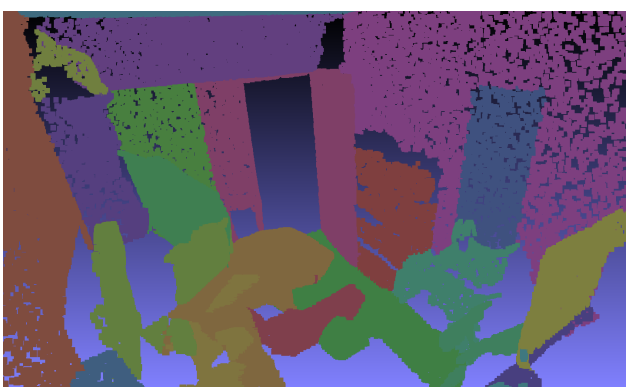

Ground-truth

Figure 4.6: Instance segmentation results for Office 22.

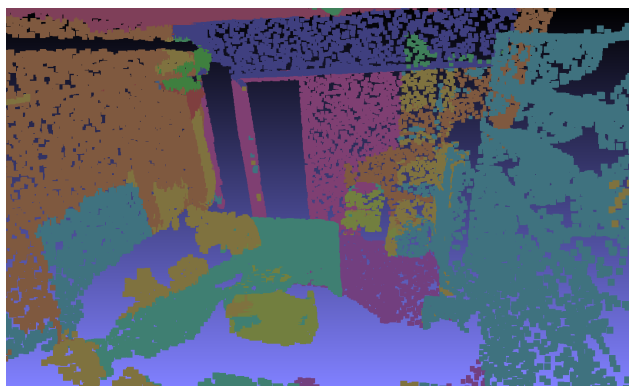

Clustering on features

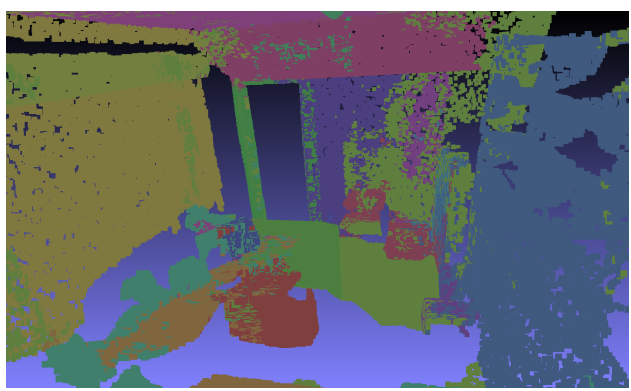

SGPN

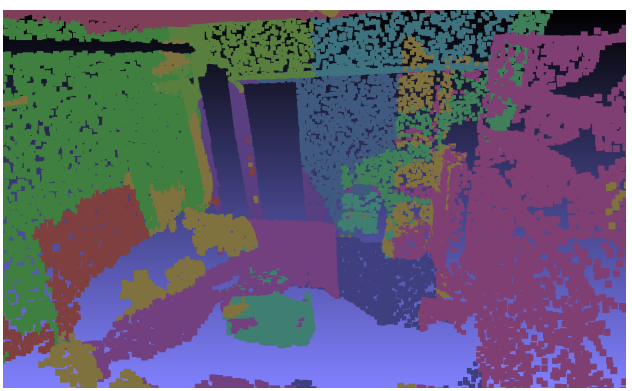

Clustering on coordinates

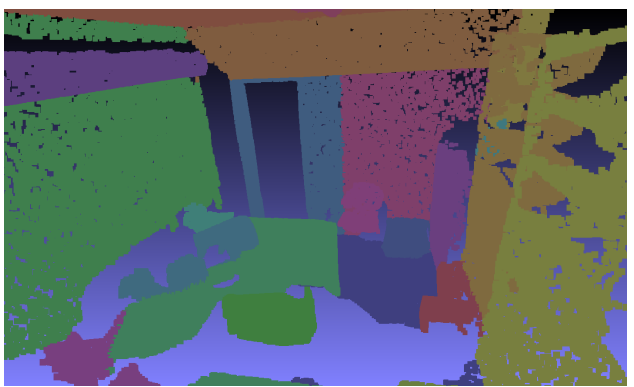

Ground-truth

Figure 4.7: Instance segmentation results for Office 31. 


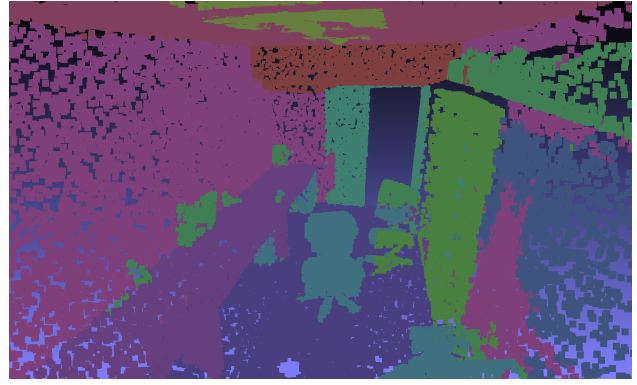

Clustering on features

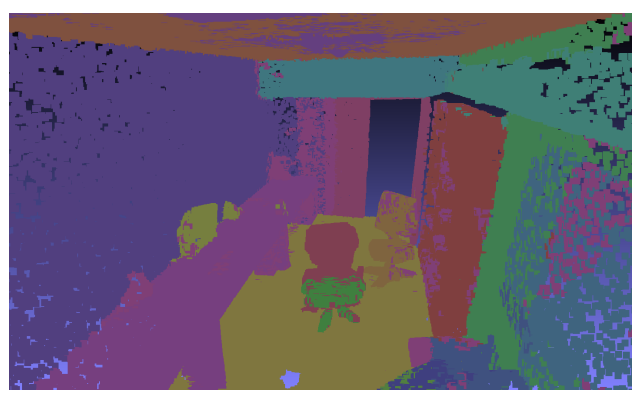

SGPN

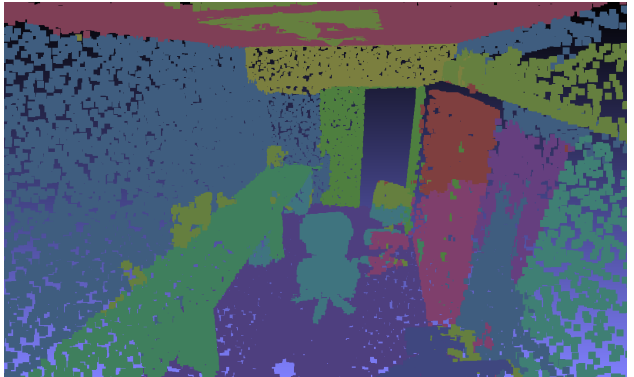

Clustering on coordinates

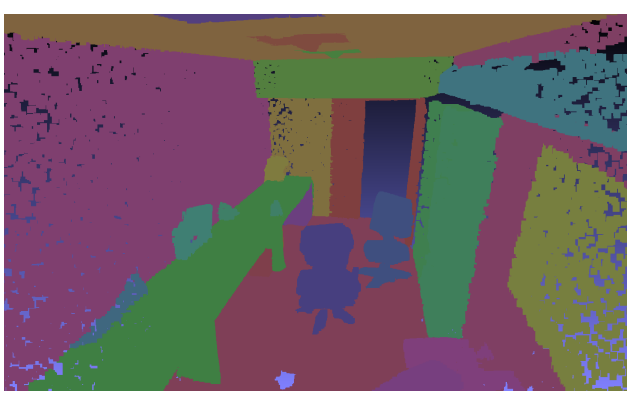

Ground-truth

Figure 4.8: Instance segmentation results for Office 29.

For the CIMS dataset, we apply our method to Rooms 601, 484, and 371, where we perform spectral clustering with point features and point coordinates to obtain the instances. The results are shown in Figure 4.9.

As expected, clustering on point features is able to better capture the similarity between points of different categories. In the visual examples, most of the points of the same category are assigned to the same cluster, i.e., instance. This is useful as, if we look at the second row and second column in Figure 4.9, clustering on point coordinates has split the fireplace at the center of the image into two clusters. In the same image, tables and chairs have been put into one cluster. We do not notice such errors in the result obtained by clustering with point features. Inclusion of all instances of one category into one instance can easily be corrected by user input. However, if two categories are termed as one instance, it is hard to rectify this error using user input. 

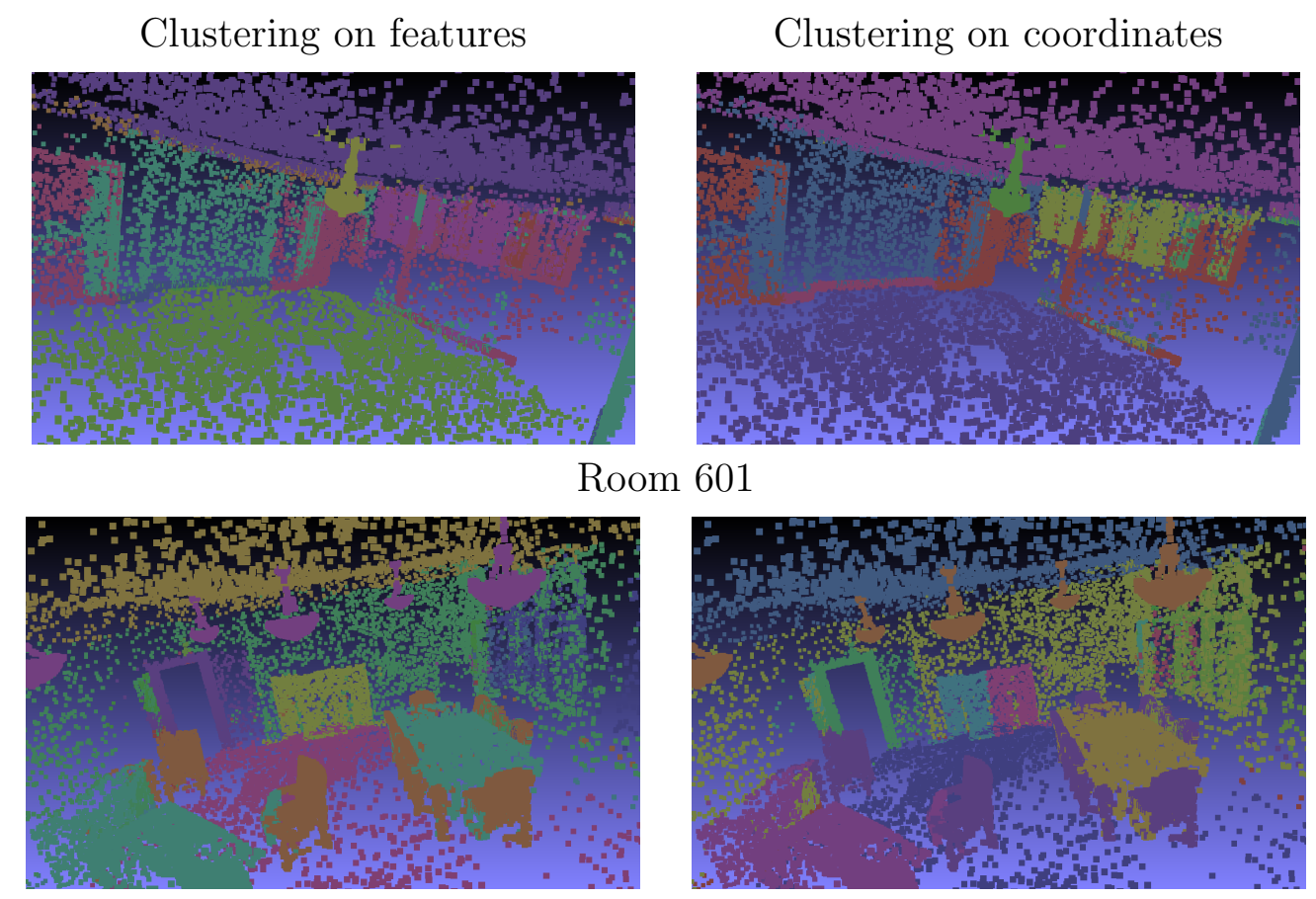

Room 484

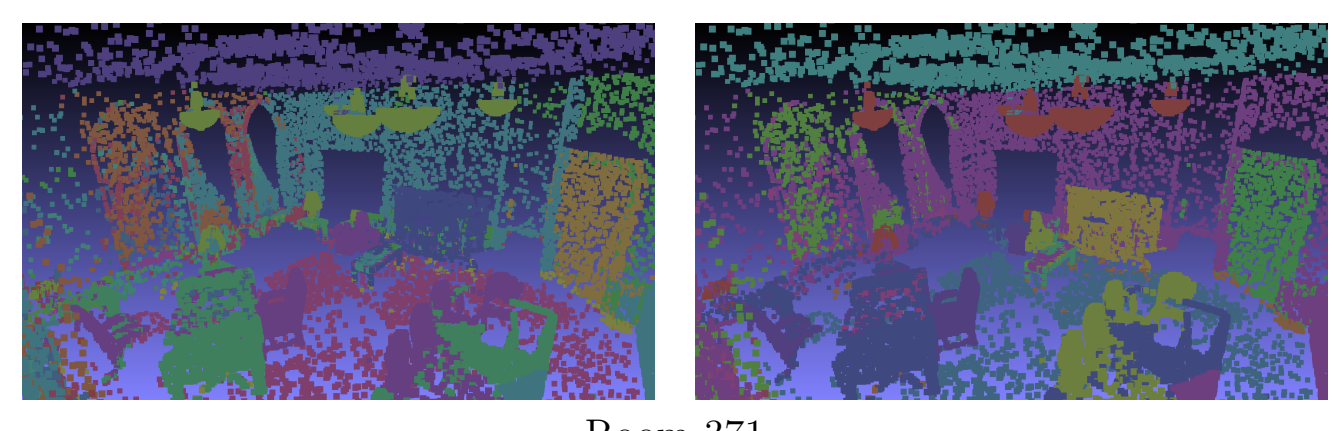

Room 371

Figure 4.9: Instance segmentation results on CIMS dataset.

\subsection{Constrained Clustering Evaluation (Quantitative and Qualitative)}

After finding instance labels, the user can optionally improve the results by guiding the clustering with constraints. To demonstrate the benefit of the constrained clustering method, we select two rooms from the S3DIS dataset. Office 2 is a standard room with the typical one ceiling per room and an accurate instance segmentation 
result. Office 8 is an irregular room which is shaped like a hallway, has three ceilings, and an inaccurate instance segmentation result.

Tables 4.12 and 4.13 present the results of instance segmentation on Office 2 and 8 with the point measure. Tables 4.14 and 4.15 present the results with the bounding box measure. In both cases, the measure is $\mathrm{AP} \%$ with IOU of 0.5.

For both rooms, our spectral clustering results on features are already better than SGPN. We further improve the results with user input. Here, we have a choice of using features or coordinates for clustering, depending on the configuration of the objects. In some cases, coordinates can lead to a better clustering when objects are already spatially separated from each other. In other more complex cases, features provide better results.

For example, in Table 4.14 for Office 2, we can analyze that specifying the correct number of clusters for clustering with coordinates already gives us better results without the need of constraints. Moreover, in Table 4.15, spectral clustering on coordinates provides an AP\% of 24.7, whereas spectral clustering on features provides an $\mathrm{AP} \%$ of only 9.09. However, as seen in most values in the tables, spectral clustering on features always outperforms spectral clustering on coordinates with user assistance.

\begin{tabular}{|c|c|c|c|c|c|c|c|c|c|c|c|c|}
\hline & Mean & ceiling & floor & wall & beam & column & window & door & table & chair & bookcase & board \\
\hline SGPN & 53.03 & 100 & 100 & 43.33 & 100 & 0 & 0 & 100 & 40 & 0 & 100 & 0 \\
\hline Features & 69.09 & 100 & 100 & 30 & 100 & 0 & 100 & 100 & 30 & 0 & 100 & 100 \\
\hline User specified number of clusters on features & 69.5 & 100 & 100 & 15 & 100 & 0 & 100 & 100 & 30 & 20 & 100 & 100 \\
\hline Cannot link constraints on features & 75.6 & 100 & 100 & 30 & 100 & 0 & 100 & 100 & 30 & 72 & 100 & 100 \\
\hline Coordinates & 50.9 & 100 & 100 & 30 & 0 & 0 & 100 & 0 & 0 & 30 & 100 & 100 \\
\hline User specified number of clusters on coordinates & 68.18 & 100 & 100 & 0 & 100 & 0 & 100 & 100 & 30 & 20 & 100 & 100 \\
\hline Cannot link constraints on coordinates & 74 & 100 & 100 & 0 & 100 & 0 & 100 & 100 & 30 & 84 & 100 & 100 \\
\hline
\end{tabular}

Table 4.12: Instance segmentation results on Office 2 from the S3DIS dataset. The metric is $\mathrm{AP} \%$ with IOU of 0.5 and is calculated using the point method. 


\begin{tabular}{|c|c|c|c|c|c|c|c|c|c|c|c|}
\hline & Mean & ceiling & floor & wall & beam & column & window & door & table & chair & bookcase \\
\hline SGPN & 9.9 & 0 & 0 & 24 & 60 & 0 & 0 & 0 & 0 & 15 & 0 \\
Features & 13 & 0 & 100 & 30 & 0 & 0 & 0 & 0 & 0 & 0 & 0 \\
User specified number of clusters on features & 11.53 & 0 & 100 & 12 & 0 & 0 & 0 & 0 & 0 & 3 & 0 \\
Cannot link constraints on features & 41.4 & 50 & 100 & 15 & 0 & 0 & 60 & 100 & 26 & 63.14 & 0 \\
Coordinates & 24.5 & 0 & 100 & 30 & 15 & 0 & 0 & 100 & 0 & 0 & 0 \\
User specified number of clusters on coordinates & 31.9 & 15 & 100 & 0 & 0 & 0 & 60 & 100 & 5 & 39.39 & 0 \\
Cannot link constraints on coordinates & 41.3 & 43.33 & 100 & 24 & 0 & 0 & 60 & 100 & 26 & 60 & 0 \\
\hline
\end{tabular}

Table 4.13: Instance segmentation results on Office 8 from the S3DIS dataset. The metric is AP\% with IOU of 0.5 and is calculated using the point method.

\begin{tabular}{|c|c|c|c|c|c|c|c|c|c|c|c|c|}
\hline & Mean & ceiling & floor & wall & beam & column & window & door & table & chair & bookcase & board \\
\hline SGPN & 10.03 & 10.1 & 6.49 & 5.68 & 10.1 & 0 & 22.72 & 9.09 & 5.34 & 15.15 & 7.57 & 18.18 \\
Features & 52.3 & 90.9 & 90.9 & 0 & 90.9 & 0 & 90.9 & 90.9 & 30.3 & 0 & 90.9 & 0 \\
User specified number of clusters on features & 57.85 & 90.9 & 90.9 & 0 & 90.9 & 0 & 90.9 & 90.9 & 90.9 & 0 & 90.9 & 0 \\
Cannot link constraints on features & $\mathbf{6 6 . 1 1}$ & 90.9 & 90.9 & 0 & 90.9 & 0 & 90.9 & 90.9 & 90.9 & 90.9 & 90.9 & 0 \\
Coordinates & 33.05 & 90.9 & 90.9 & 0 & 0 & 0 & 90.9 & 0 & 0 & 0 & 90.9 & 0 \\
User specified number of clusters on coordinates & $\mathbf{6 6 . 1 1}$ & 90.9 & 90.9 & 0 & 90.9 & 0 & 90.9 & 90.9 & 90.9 & 90.9 & 90.9 & 0 \\
Cannot link constraints on coordinates & 53.71 & 90.9 & 90.9 & 0 & 0 & 0 & 90.9 & 90.9 & 45.45 & 90.9 & 90.9 & 0 \\
\hline
\end{tabular}

Table 4.14: Instance segmentation results on Office 2 from the S3DIS Dataset. The metric is $\mathrm{AP} \%$ with IOU of 0.5 and is calculated using the bounding box method.

\begin{tabular}{|c|c|c|c|c|c|c|c|c|c|c|c|}
\hline & Mean & ceiling & floor & wall & beam & column & window & door & table & chair & bookcase \\
\hline SGPN & 7.8 & 0 & 2.75 & 12.98 & 10.1 & 0 & 22.72 & 0 & 11.36 & 18.18 & 0 \\
Features & 9.09 & 0 & 90.9 & 0 & 0 & 0 & 0 & 0 & 0 & 0 & 0 \\
User specified number of clusters on features & 9.09 & 0 & 90.9 & 0 & 0 & 0 & 0 & 0 & 0 & 0 & 0 \\
Cannot link constraints on features & $\mathbf{5 1 . 5}$ & 30.3 & 90.9 & 0 & 30.3 & 0 & 90.9 & 90.9 & 90.9 & 90.9 & 0 \\
Coordinates & 24.7 & 0 & 90.9 & 0 & 0 & 0 & 90.9 & 90.9 & 0 & 0 & 0 \\
User specified number of clusters on coordinates & 36.36 & 30.3 & 90.9 & 0 & 0 & 0 & 90.9 & 90.9 & 30.3 & 30.3 & 0 \\
Cannot link constraints on coordinates & 48.48 & 30.3 & 90.9 & 0 & 45.45 & 0 & 90.9 & 90.9 & 45.45 & 90.9 & 0 \\
\hline
\end{tabular}

Table 4.15: Instance segmentation results on Office 8 from the S3DIS dataset. The metric is $\mathrm{AP} \%$ with IOU of 0.5 and is calculated using the bounding box method.

We calculated the improvement that our constrained clustering method offers in terms of mPrec and mRecall to better compare with recent methods. The only available results per room are from SGPN. Thus, we compare the Office 2 and Office 8 results with our method as shown in Table 4.16 and Table 4.17. 


\begin{tabular}{|c|c|c|}
\hline & mPrec & mRecall \\
\hline Ours (features) & 38.58 & 49.77 \\
Ours (coordinates) & 40.01 & 50.47 \\
SGPN & 38.28 & 39.93 \\
\hline
\end{tabular}

Table 4.16: Instance segmentation results on the S3DIS dataset for Office 8. The metrics are mean precision and mean recall with $\mathrm{IoU}$ threshold of 0.5 , which are calculated using the point set method.

\begin{tabular}{|c|c|c|}
\hline & mPrec & mRecall \\
\hline Ours (features) & 75.24 & 75.90 \\
Ours (coordinates) & 78.32 & 77.25 \\
SGPN & 68.65 & 75.75 \\
\hline
\end{tabular}

Table 4.17: Instance segmentation results on the S3DIS dataset for Office 2. The metrics are mean precision and mean recall with $\mathrm{IoU}$ threshold of 0.5 , which are calculated using the point set method.

Figures 4.11 and 4.12 show instance segmentation results from our spectral clustering method as well as the results from SGPN and ground-truth. The different colors represent different instance labels.

As visible in these results, because SGPN processes results in blocks, the global visualization of points does not look as clean as it should. On the other hand, we process the entire room by separating it into different categories and the points in each category are assigned instance labels without breaking them apart.

The results for chairs and tables for our spectral clustering method on point features and coordinates is poor. However, after user input, distinct chairs and tables can be easily identified.

The number of times a user adds constraints depends on the number of clusters the user identifies. For example, in the case of chairs for Office 2, the user identifies that there should be 5 clusters for 5 chairs. The method then allows the user to enter 5 constraints. In the case of walls, the user identifies 4 clusters and can input 4 constraints. For each input, the user can circle any number of points twice, and the 
method adds the corresponding constraints to the matrix. In total, with 9 constraints, the user has achieved an AP\% of 75.6.

One benefit of the constrained clustering based on the similarity matrix is that the user often does not have to enter a constraint for each cluster. For example, in the case of Office 2, for chairs, we could get from an AP\% of 20 to 75 with 4 user inputs instead of 5 . In this particular case, the user spent 1 minute and 25 seconds to cluster five chair instances. In Office 2, for walls, we could get from an AP\% of 15 to 30 with just 2 constraints instead of 4 . In this case, the user spent 2 minutes and 54 seconds to cluster the walls. This example is shown in Figure 4.10.

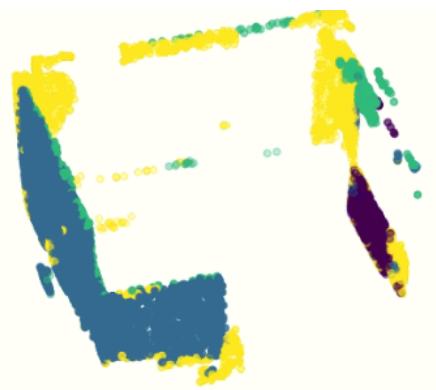

Clustering on features

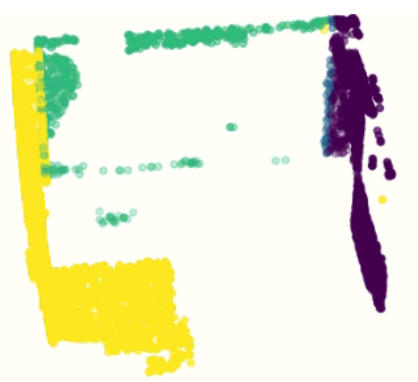

Constrained clustering after two user inputs

Figure 4.10: Constrained clustering results for walls of Office 2.

The time and effort the user spends on constrained clustering depends on the size of the object as well as the technique the user chooses. For example, even though there are more chairs than walls in the above example, walls have more points than chairs and so clustering the chairs takes less time. With regards to the technique, if the user chooses to separate object one at a time instead of multiple objects in one go, this would also increase the time spent on this process.

While the constrained clustering process may look like manual segmentation, constrained clustering offers the benefit of knowing the semantic and instance labels prior to the process. Another distinction is that in manual segmentation, the user has to separate each object from the other objects one by one, whereas in constrained clustering the user may choose to select more than one instance at a time to identify that particular instance as a separate object, as seen in Figure 3.7. 


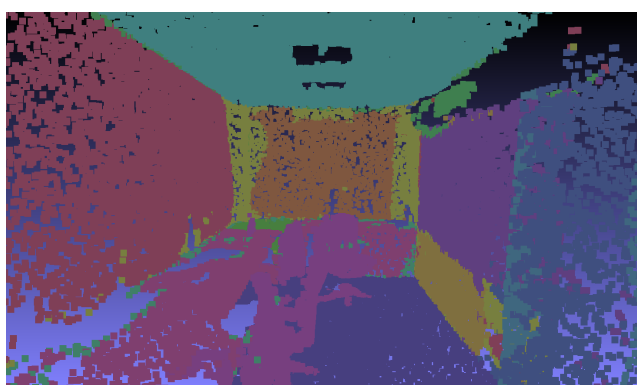

Clustering on features

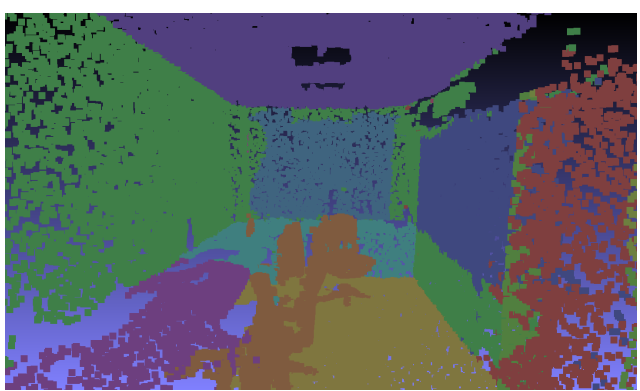

Clustering on coordinates

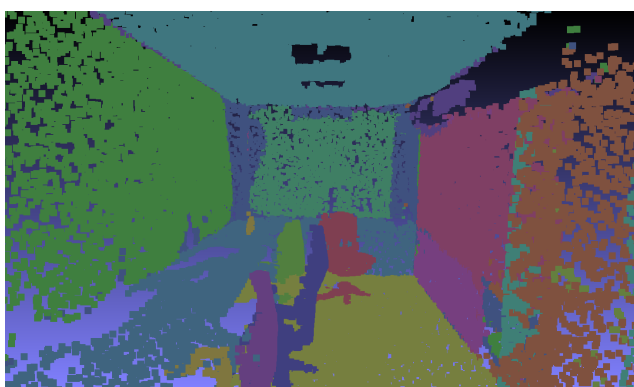

Constrained clustering on features

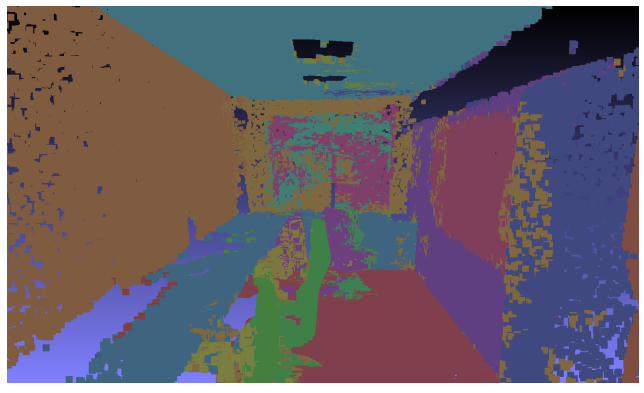

SGPN

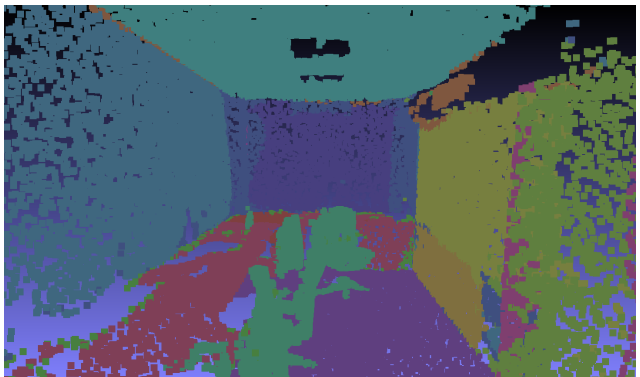

With manual \# clusters

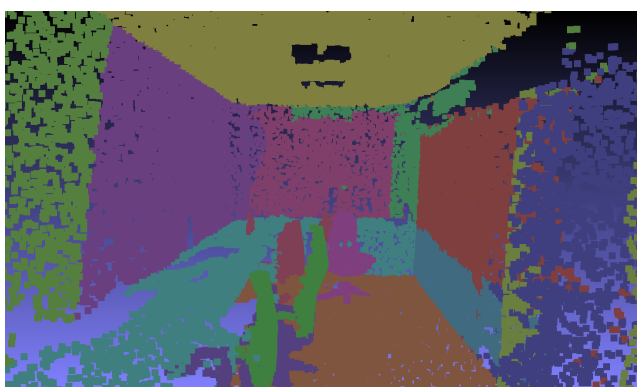

With manual \# clusters

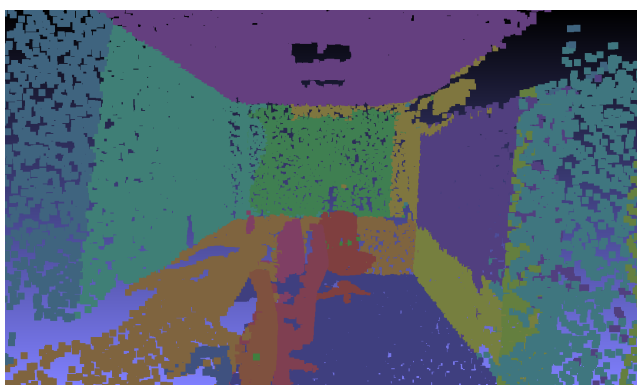

Constrained clustering on coordinates

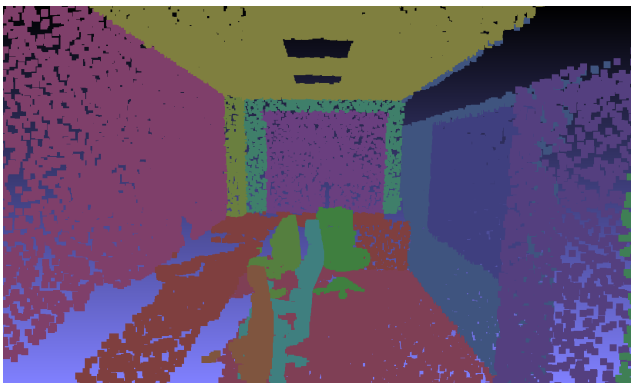

Ground-truth

Figure 4.11: Office 2 results for instance segmentation. The second columns on the first and second rows show the result on the left improved by manually providing the number of clusters. 


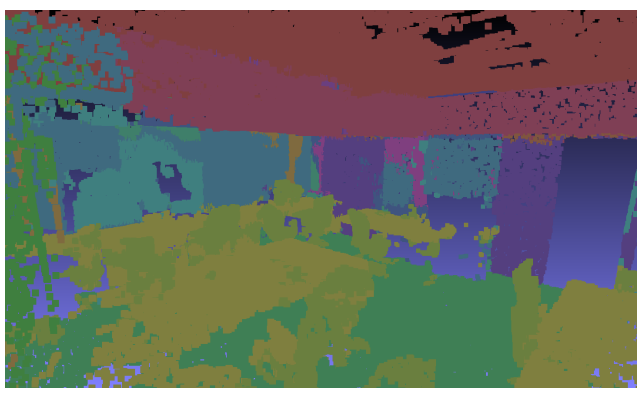

Clustering on features

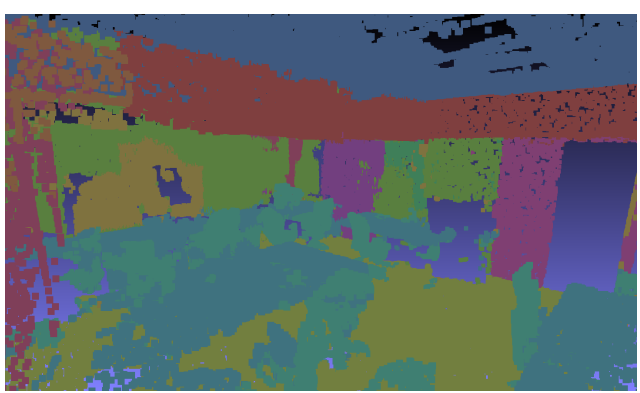

Clustering on coordinates

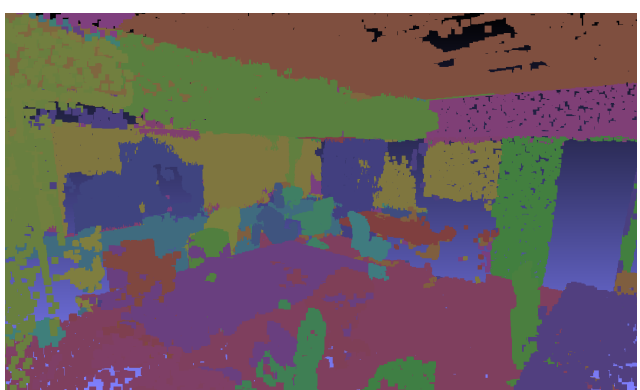

Constrained clustering on features

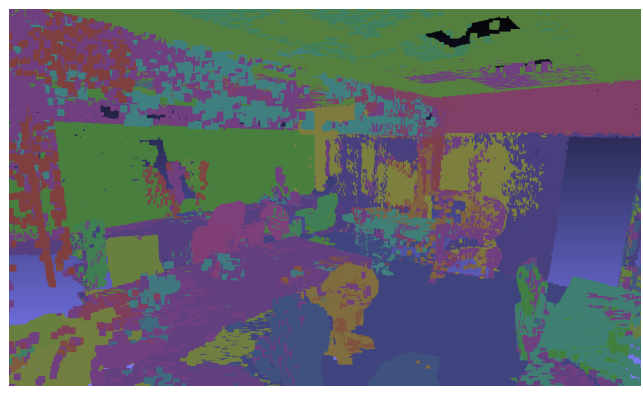

SGPN

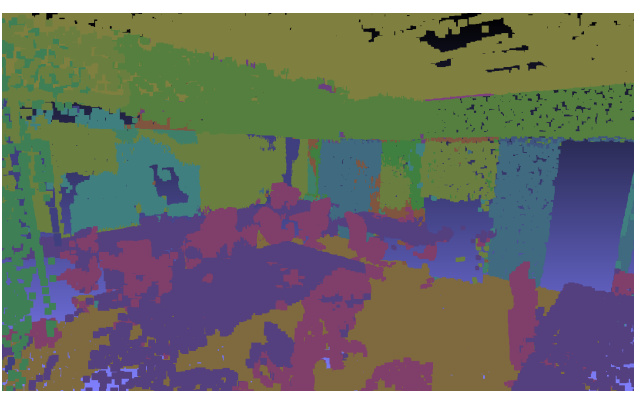

With manual \# clusters

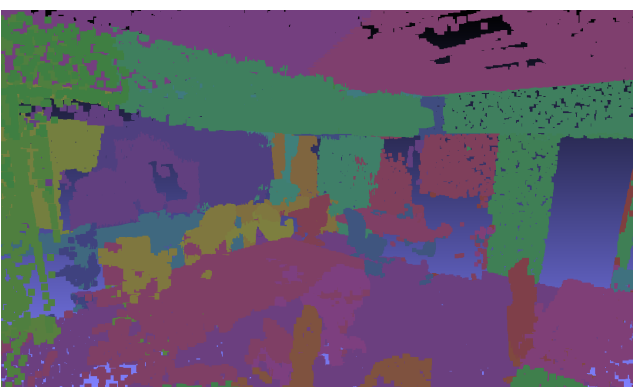

With manual \# clusters

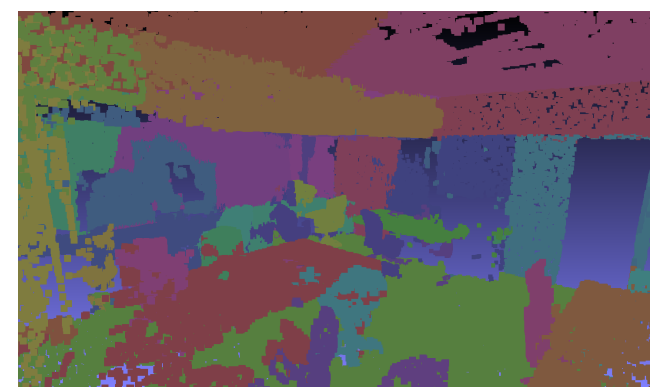

Constrained clustering on coordinates

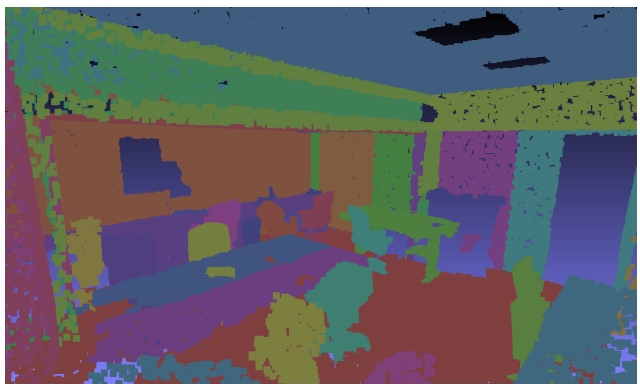

Ground-truth

Figure 4.12: Office 8 results for instance segmentation. The second columns on the first and second rows show the result on the left improved by manually providing the number of clusters.

Next, we evaluate the results on the CIMS dataset. In this case, we cluster using 
point features and point coordinates. The user may decide to specify the number of clusters as well as perform constrained clustering. The results are shown in Figures 4.13 and 4.14 .

The result for semantic segmentation of walls for both the rooms is not up to standard and therefore the instance segmentation results are poor as well. Just like in S3DIS, the instance segmentation results for chairs and tables need user-assisted constrained clustering that helps to identify the different instances.

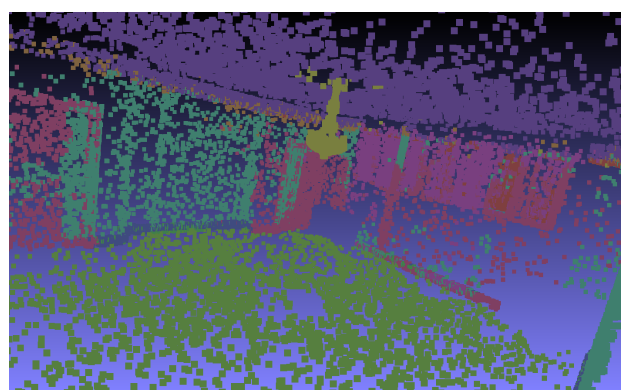

Clustering on features

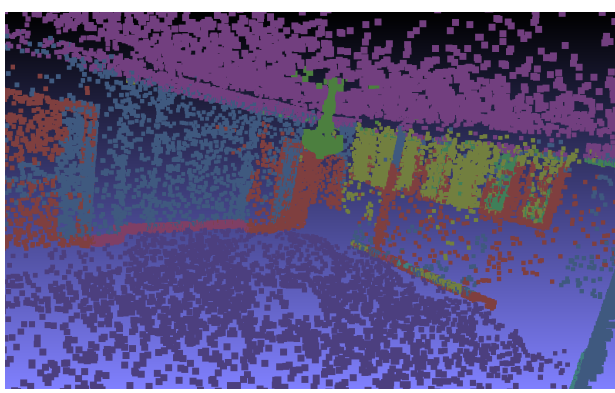

Clustering on coordinates

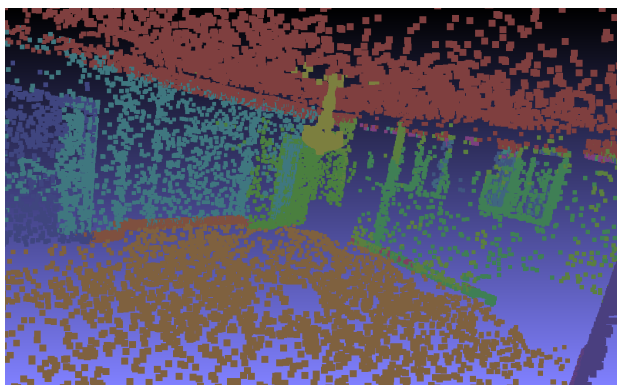

Constrained clustering on features

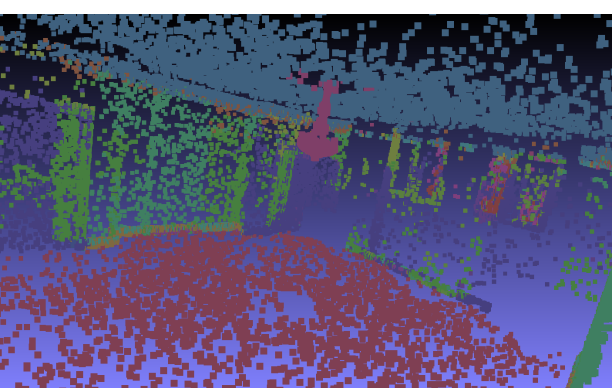

With manual \# clusters

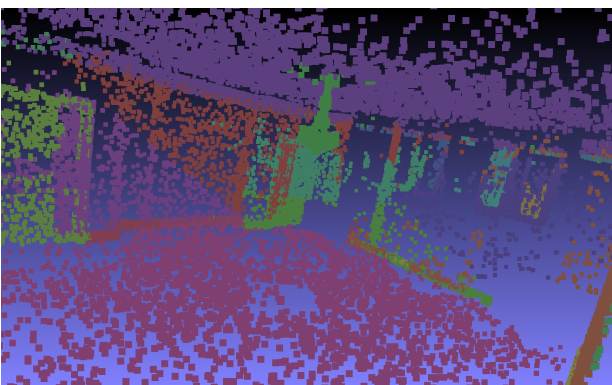

With manual \# clusters

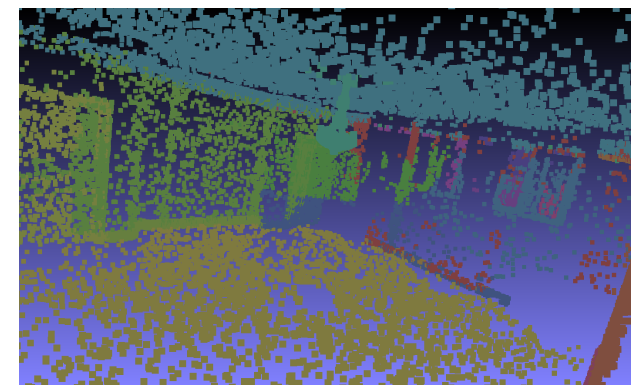

Constrained clustering on coordinates

Figure 4.13: $601 \mathrm{~S}$ results for instance segmentation. The second columns on the first and second rows show the result on the left improved by manually providing the number of clusters. 
In Room 484S, there are around 5-6 light fixtures present. By providing the number of clusters to the method for point coordinates, we are able to clearly distinguish each instance from the other without any constrained clustering (second row, second column in Figure 4.14). This is because the distance between all the light fixtures provides sufficient separation between the different objects.

However, we cannot completely rely on the coordinates. We see in Figure 4.14 that the fireplace in the image in the second row and first column and the wall in the image in the second row and second column have been broken into two different clusters whereas they are single objects. As the point features indicate the similarity between points, we see that when clustering with point features (first row, first column), this mistake is avoided.

Similarly, with constrained clustering (third row), we obtain better results for clustering with point features than with coordinates, especially in the categories door, chair, and table. 


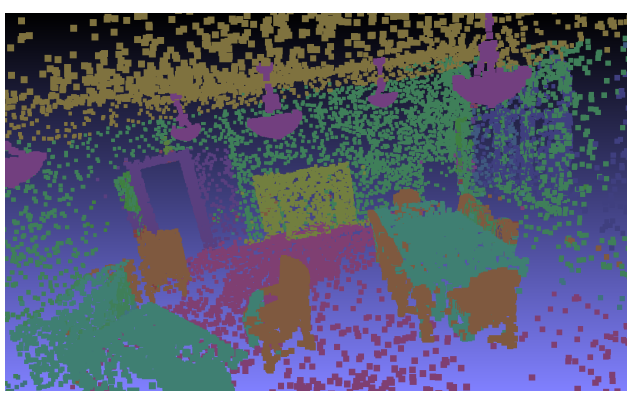

Clustering on features

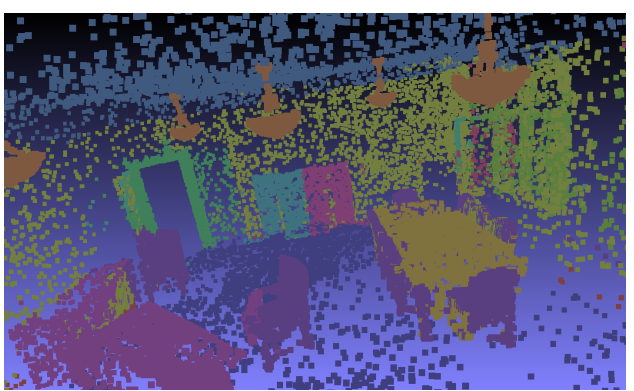

Clustering on coordinates

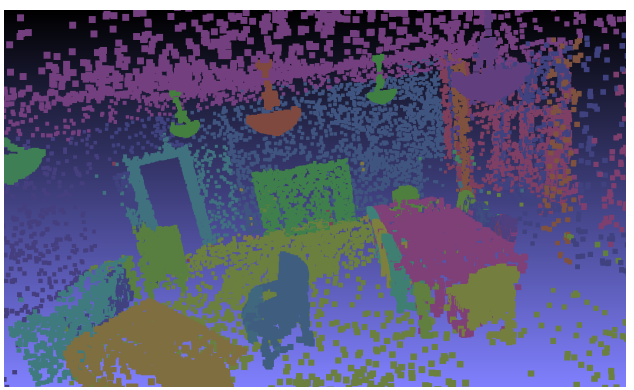

Constrained clustering on features

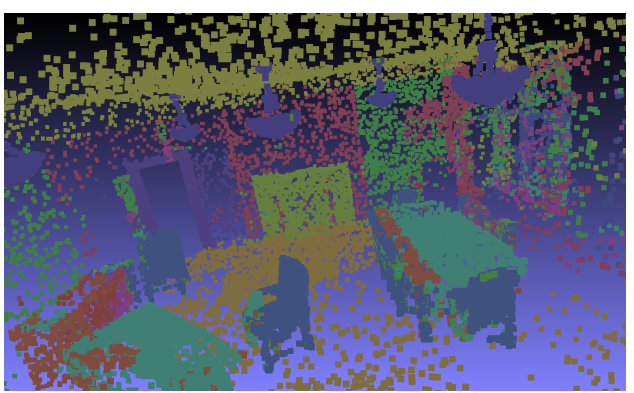

With manual \# clusters

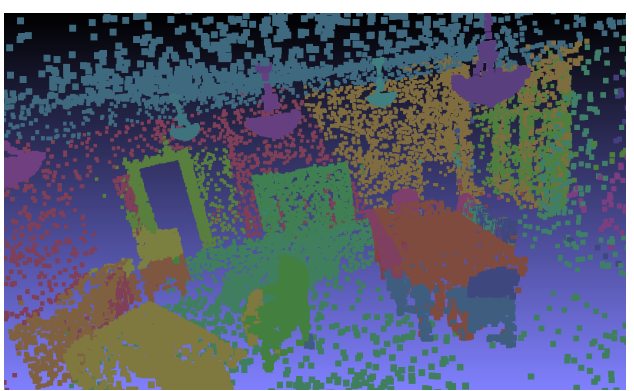

With manual \# clusters

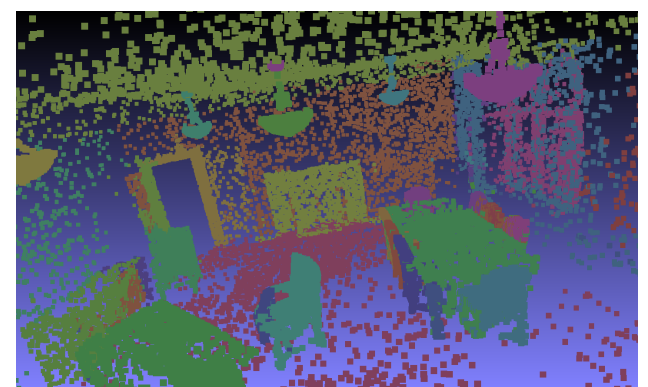

Constrained clustering on coordinates.

Figure 4.14: Room 484S results for instance segmentation. The second columns on the first and second rows show the result on the left improved by manually providing the number of clusters.

As demonstrated in Figure 3.9 in Chapter 3, constrained clustering involves manual work but it has its benefits. Using constrained clustering, we identify a set of points that belong to a different cluster that they are assigned to. Doing so results in those points being excluded from that instance/cluster but also from other unrelated clusters. 


\subsection{Execution time}

We train our model on an NVIDIA GP104GL [Quadro P4000] GPU with CUDA version 9.1. For both of the datasets, the size of the rooms can range from 50 thousand points to 3 million points. Table 4.18 shows the training time and testing time required by our method, split between time required for semantic segmentation and instance segmentation. The training time for the S3DIS dataset comprises training for 200 epochs on 5 train areas. Each area contains about 40 rooms. The training time for the CIMS dataset comprises training for 100 epochs for about 36 rooms.

The test time for S3DIS semantic segmentation is for a room with 1,508,952 points, where the attributes include coordinates, colors, and semantic labels. The test time for S3DIS instance segmentation is with respect to a room with 172,032 points and 131 features, including point coordinates. The test time for CIMS semantic segmentation is for a room with 110,592 points with coordinates, colors, and semantic labels. The test time for CIMS instance segmentation is with respect to a room with 65,536 points and 131 features, including point coordinates.

\begin{tabular}{|l|c|c|c|}
\hline & Training Time & Semantic Segmentation Testing & Instance Segmentation Testing \\
\hline S3DIS & approximately 3 days & 22.3 seconds & 1968.3 seconds \\
CIMS & 15.58 hours & 15.6 seconds & 817.3 seconds \\
\hline
\end{tabular}

Table 4.18: Execution time analysis for our method. 


\section{Chapter 5}

\section{Conclusion}

In this chapter, we conclude the discussion of our method, provide an analysis of limitations of our method, and discuss ideas for prospective work.

\subsection{Discussion}

In this thesis, we introduced a method for instance segmentation on point clouds of indoor scenes without the use of instance labels. Our work builds up on PointNet. We modify their network for semantic segmentation to output point features that reflect the similarity that points have with each other when they belong to the same category. We call this network PointSimNet. PointSimNet produces semantic labels and a feature matrix of 128 features per point. This feature matrix along with the semantic labels is used to perform instance segmentation.

Instance segmentation is performed using the spectral clustering method. Here, we have the option of using the feature matrix or the coordinates of the points in the point cloud. To perform spectral clustering, we form a matrix by calculating the distance between all points that belong to the same category. This distance can be the distance between point features or point coordinates. Then, we convert the distance matrix into a similarity matrix by passing it through a Gaussian kernel. Once we find the similarity matrix, we use the eigengap heuristic to find the optimal number of clusters for each category. The spectral clustering method performs instance segmentation on the similarity matrix using the optimal number of clusters.

Depending on the user satisfaction on the instance segmentation result, the user can further perform constrained clustering. Here, the user specifies the number of clusters as well as points that cannot be linked together. When the user adds a 
cannot-link constraint, the method increases the distance between points or equivalently decreases their similarity with each other. This is beneficial as the users can themselves tune the clustering to the level of accuracy that they require.

Our results of the instance segmentation method have lower AP than the methods that require instance labels for training such as SGPN [50] and other concurrent methods $[21,24]$. However, when considering an alternative definition of the evaluation measure, we outperform SGPN by 20 AP percentage points without using instance labels.

An advantage of our method is that we process the entire room at once while SGPN processes it block by block. This results in visual artifacts in SGPN's results, where chunks of categories mix with each other on a global level. Our results look visually cleaner. Another advantage of our approach is that we mainly use the information provided by semantic labels to perform instance segmentation, whereas SGPN requires instance labels for training.

A typical application of our method is to help with building information modeling of heritage buildings. As BIMs for major renovation projects related to heritage buildings need to be created before starting renovations, a lot of user effort and time is required for the creation of such models. When there is no information given about the building except for point clouds, we can make use of our method to help building a BIM. When we have an unlabeled room and pass it through PointSimNet, we obtain the output in the form of semantic labels and point features. These then help us to produce instances for all the objects present in the room. This gives way to a BIM that can be used for tracking assets and in maintenance and operations of the heritage building. The user input can be optionally added to reach a higher level of accuracy.

\section{$5.2 \quad$ Limitations}

There are some drawbacks to our method which are listed below:

- The instance segmentation process depends on the results of semantic segmentation. If semantic segmentation results are not up to standard, for example, if a chair has been categorized as a table, then the accuracy of the instance segmentation results will also be decreased.

- Our spectral clustering method is not able to properly separate certain categories of objects, such as chairs and tables, even with Euclidean distances. 
The reason being that most of these objects are clustered very close together. However, this problem can be easily solved with constrained clustering in just a few tries. This indicates that it should be possible to improve the automatic instance segmentation for these categories with a minimum of additional data.

- In order for constrained clustering to achieve high accuracy on categories such as wall or bookshelves, the user has to be familiar with the dataset to make appropriate changes. This is because not all walls are split into four corners in the dataset, and some bookshelves are split into two, even though there is no space between the bookshelves and they are placed along the same wall. This indicates that the existing datasets are not entirely accurate and could be improved for training more accurate models.

- In addition, constrained clustering works very well for some categories but for others there is not much the user can do. For example, if the user can identify two objects as being separate due to the distance between them, they can easily be separated with constraints. However, for the categories column and board, either the semantic segmentation results are not up to standard, or the user is unable to identify the different instances due to their confusing distribution/structure.

\subsection{Future work}

We discuss the ideas for future work in this section.

One idea for future work that can increase the performance of the method is to use both point features and coordinates to perform the clustering of instances. We observed that, while in most of the cases features provide a better instance segmentation result, euclidean distances can be quite useful to segment certain categories. Thus, an improvement to the method would use both attributes to find the instances. This could be achieved by combining the feature distances and euclidean distances into one matrix and using that to perform clustering. However, this requires to select a proper weighting between the two attributes, which would be linked to the quality of the results.

Another direction to experiment with would be to perform clustering using other methods such as region growing clustering methods, which consider both the similarity 
matrix and spatial proximity of points when performing the clustering. This could be an additional step after we have found the initial instance labels using spectral clustering. This would solve the issue of separating chairs and tables and other objects by making use of the information of instances as well as coordinate distances.

Finally, we could also investigate the use of semi-supervised methods for improving instance segmentation results across different rooms, which would reduce even more the amount of work required from uses. In this scenario, adding user guidance for the segmentation of one room would also improve the results of another room. This may be possible since methods for segmentation of individual objects have achieved such a goal [49]. 


\section{List of References}

[1] N. M. A, Neural networks and Deep Learning. Determination press San Francisco, CA, 2015, vol. 2018.

[2] E. Ahmed, A. Saint, A. E. R. Shabayek, K. Cherenkova, R. Das, G. Gusev, D. Aouada, and B. Ottersten, "Deep learning advances on different 3d data representations: A survey." CoRR, 2018.

[3] F. Andrea, S. Yoram, S. Fei, and M. Jitendra, "Learning globally-consistent local distance functions for shape-based image retrieval and classification," in 2007 IEEE 11th International Conference on Computer Vision. IEEE, 2007, pp. 1-8.

[4] I. Armeni, O. Sener, A. R. Zamir, H. Jiang, I. Brilakis, M. Fischer, and S. Savarese, "3d semantic parsing of large-scale indoor spaces," in Proceedings of the IEEE International Conference on Computer Vision and Pattern Recognition, 2016.

[5] S. Asafi and D. Cohen-Or, "Constraints as features," in Proceedings of the IEEE Conference on Computer Vision and Pattern Recognition, 2013, pp. 1634-1641.

[6] Autodesk, Inc., "Building information modeling," San Rafael, CA, USA, Tech. Rep., 2002.

[7] H. B. Barlow, "Unsupervised learning," Neural computation, vol. 1, no. 3, pp. 295-311, 1989.

[8] A. Bellet, A. Habrard, and M. Sebban, "A survey on metric learning for feature vectors and structured data," arXiv preprint arXiv:1306.6709, 2013.

[9] Y. Bo, W. Jianan, C. Ronald, H. Qingyong, W. Sen, M. Andrew, and T. Niki, "Learning object bounding boxes for 3d instance segmentation on point clouds," in Advances in Neural Information Processing Systems, 2019, pp. 6740-6749.

[10] M. Botsch, L. Kobbelt, M. Pauly, P. Alliez, and B. Levy, Polygon Mesh Processing. CRC Press, 2010, ch. 1.

[11] S. Chopra, R. Hadsell, and Y. LeCun, "Learning a similarity metric discriminatively, with application to face verification," in 2005 IEEE Computer Society Conference on Computer Vision and Pattern Recognition (CVPR'05), vol. 1. IEEE, 2005, pp. 539-546. 
[12] M. Christopher, R. Prabhakar, and S. Hinrich, "Introduction to information retrieval," Natural Language Engineering, vol. 16, no. 1, pp. 100-103, 2010.

[13] F. R. K. Chung, Spectral Graph Theory. American Mathematical Society, 1997.

[14] S. J. Dyssel, S. Yongbin, C. Wiley, and G. S. W, "Visualization and labeling of point clouds in virtual reality," in SIGGRAPH Asia 2017 Posters, 2017, pp. 1-2.

[15] V. Estivill-Castro, "Why so many clustering algorithms: a position paper," SIGKDD Explorations, vol. 4, pp. 65-75, 2002.

[16] P. Follmann and R. König, "Oriented boxes for accurate instance segmentation," arXiv preprint arXiv:1911.07732, 2019.

[17] S. Gold, A. Rangarajan, C.-P. Lu, S. Pappu, and E. Mjolsness, "New algorithms for 2d and 3d point matching: Pose estimation and correspondence," Pattern recognition, vol. 31, no. 8, pp. 1019-1031, 1998.

[18] L. Gonzales. (2019) An in-depth look at pointnet. [Online]. Available: https: //medium.com/@luis_gonzales/an-in-depth-look-at-pointnet-111d7efdaa1a

[19] I. Goodfellow, Y. Bengio, A. Courville, and Y. Bengio, Deep learning. MIT press Cambridge, 2016, vol. 1.

[20] E. Grilli, F. Menna, and F. Remondino, "A review of point clouds segmentation and classification algorithms," The International Archives of Photogrammetry, Remote Sensing and Spatial Information Sciences, vol. 42, p. 339, 2017.

[21] L. Han, T. Zheng, L. Xu, and L. Fang, "Occuseg: Occupancy-aware 3d instance segmentation," in Proceedings of the IEEE/CVF Conference on Computer Vision and Pattern Recognition, 2020, pp. 2940-2949.

[22] S. Hang, M. Subhransu, K. Evangelos, and L.-M. Erik, "Multi-view convolutional neural networks for 3d shape recognition," in Proceedings of the IEEE international conference on computer vision, 2015, pp. 945-953.

[23] J. Hou, A. Dai, and M. Nießner, "3d-sis: 3d semantic instance segmentation of rgb-d scans," 2019, pp. 4421-4430.

[24] L. Jiang, H. Zhao, S. Shi, S. Liu, C.-W. Fu, and J. Jia, "Pointgroup: Dual-set point grouping for 3d instance segmentation," in Proceedings of the IEEE/CVF Conference on Computer Vision and Pattern Recognition, 2020, pp. 4867-4876.

[25] K. Justin, W. Lipo, R. Jai, and L. Tchoyoson, "Deep learning applications in medical image analysis," IEEE Access, vol. 6, pp. 9375-9389, 2017.

[26] K. Kamvar, S. Sepandar, K. Klein, D. Dan, M. Manning, and C. Christopher, "Spectral learning," in International Joint Conference of Artificial Intelligence. Stanford InfoLab, 2003.

[27] J. Kawale and D. Boley, "Constrained spectral clustering using 11 regularization," in Proceedings of the 2013 SIAM International Conference on Data Mining. SIAM, 2013, pp. 103-111. 
[28] R. Khandelwal. (2019) Evaluating performance of an object detection model. [Online]. Available: https://towardsdatascience.com/ evaluating-performance-of-an-object-detection-model-137a349c517b

[29] H. S. Koppula, A. Anand, T. Joachims, and A. Saxena, "Semantic labeling of 3d point clouds for indoor scenes," in Advances in neural information processing systems, 2011, pp. 244-252.

[30] A. Krizhevsky, I. Sutskever, and G. Hinton, "Imagenet classification with deep convolutional neural networks," Advances in neural information processing systems, 2012.

[31] W. Liu, J. Sun, W. Li, T. Hu, and P. Wang, "Deep learning on point clouds and its application: A survey," Sensors, vol. 19, no. 19, p. 4188, 2019.

[32] C. D. Manning, P. Raghavan, and H. Schütze, Introduction to Information Retrieval. MIT Press, 2009.

[33] Matplotlib. (2012) Lasso selector. [Online]. Available: https://matplotlib.org/3. 1.1/gallery/widgets/lasso_selector_demo_sgskip.html

[34] B. Michael and G. Fredric, "The relationship between recall and precision," Journal of the American society for information science, vol. 45, no. 1, pp. 1219, 1994.

[35] K. Nikhil and S. Eder, Deep Learning with Python. Springer, 2017, vol. 1.

[36] Pham, Quang-Hieu, T. Nguyen, B.-S. Hua, G. Roig, and S.-K. Yeung., "Jsis3d: Joint semantic-instance segmentation of $3 \mathrm{~d}$ point clouds with multi-task pointwise networks and multi-value conditional random fields," 2019, pp. 8827-8836.

[37] C. R. Qi, W. Liu, C. Wu, H. Su, and L. J. Guibas, "Frustum pointnets for 3d object detection from rgb-d data," Proceedings of the IEEE Conference on Computer Vision and Pattern Recognition, pp. 918-927, 2018.

[38] C. R. Qi, H. Su, K. Mo, and L. J. Guibas, "Pointnet: Deep learning on point sets for 3d classification and segmentation," in Proceedings of the IEEE conference on computer vision and pattern recognition, 2017, pp. 652-660.

[39] C. R. Qi, L. Yi, H. Su, and L. J. Guibas, "Pointnet++: Deep hierarchical feature learning on point sets in a metric space," in Advances in neural information processing systems, 2017, pp. 5099-5108.

[40] R. Raul, "The backpropagation algorithm," in Neural networks. Springer, 1996, pp. $149-182$.

[41] Y. Rikiya, N. Mizuho, D. R. K. Gian, and T. Kaori, "Convolutional neural networks: an overview and application in radiology," Insights into imaging, vol. 9, no. 4, pp. 611-629, 2018.

[42] O. Ronneberger, P. Fischer, and T. Brox, "U-net: Convolutional networks for biomedical image segmentation," in International Conference on Medical image computing and computer-assisted intervention. Springer, 2015, pp. 234-241. 
[43] B. Sugato, D. Ian, and W. Kiri, Constrained clustering: Advances in algorithms, theory, and applications. CRC Press, 2008.

[44] D. Surendran. (2004) Swiss roll dataset. [Online]. Available: http://people.cs. uchicago.edu/ $\operatorname{dinoj/manifold/swissroll.html~}$

[45] M. Talabis, R. McPherson, I. Miyamoto, and J. Martin, Information Security Analytics: Finding Security Insights, Patterns, and Anomalies in Big Data. Syngress, 2014.

[46] N. Vinod and H. G. E, "Rectified linear units improve restricted boltzmann machines," in ICML, 2010.

[47] U. Von Luxburg, "A tutorial on spectral clustering," Statistics and computing, vol. 17, no. 4, pp. 395-416, 2007.

[48] X. Wang, S. Liu, X. Shen, C. Shen, and J. Jia, "Associatively segmenting instances and semantics in point clouds." in Proceedings of the IEEE Conference on Computer Vision and Pattern Recognition, 2019, pp. 4096-4105.

[49] Y. Wang, S. Asafi, O. Van Kaick, H. Zhang, D. Cohen-Or, and B. Chen, "Active co-analysis of a set of shapes," ACM Transactions on Graphics (TOG), vol. 31, no. 6, pp. 1-10, 2012.

[50] W. Weiyue, Y. Ronald, H. Qiangui, and N. Ulrich, "Sgpn: Similarity group proposal network for 3d point cloud instance segmentation," in Proceedings of the IEEE Conference on Computer Vision and Pattern Recognition, 2018, pp. 2569-2578.

[51] Z. Wu, S. Song, A. Khosla, F. Yu, L. Zhang, X. Tang, and J. Xiao, "3d shapenets: A deep representation for volumetric shapes," In Proceedings of the IEEE Conference on Computer Vision and Pattern Recognition, pages 1912-1920, 2015.

[52] L. Zhao and W. Tao, "Jsnet: Joint instance and semantic segmentation of 3d point clouds." in $A A A I, 2020$, pp. 12 951-12 958.

[53] X. Zhu and A. B. Goldberg, Introduction to semi-supervised learning. Morgan \& Claypool Publishers, 2009, vol. 3, no. 1.

[54] X. J. Zhu, "Semi-supervised learning literature survey," University of WisconsinMadison Department of Computer Sciences, Tech. Rep., 2005. 